Revue d'archéologie préhistorique

\title{
La Grotte des Fées (Chatelperron, Allier) ou une interstratification « Chatelperronien-Aurignacien » illusoire. histoire des fouilles, stratigraphie et datations
}

The Grotte des Fées (Châtelperron, Allier), an illusory "ChâtelperronianAurignacian" interstratification. Excavation history, stratigraphy and dating

João Zilhão, Francesco d'Errico, Jean-Guillaume Bordes, Arnaud Lenoble, Jean-Pierre Texier et Jean-Philippe Rigaud

\section{(2) OpenEdition}

\section{Journals}

Édition électronique

URL : https://journals.openedition.org/paleo/721

DOI : $10.4000 /$ paleo. 721

ISSN : 2101-0420

Éditeur

SAMRA

Édition imprimée

Date de publication : 30 décembre 2007

Pagination : 391-432

ISSN : 1145-3370

Référence électronique

João Zilhão, Francesco d'Errico, Jean-Guillaume Bordes, Arnaud Lenoble, Jean-Pierre Texier et JeanPhilippe Rigaud, «La Grotte des Fées (Chatelperron, Allier) ou une interstratification « ChatelperronienAurignacien » illusoire. histoire des fouilles, stratigraphie et datations », PALEO [En ligne], 19 | 2007, mis en ligne le 23 avril 2009, consulté le 26 juin 2021. URL : http://journals.openedition.org/paleo/721 ; DOI : https://doi.org/10.4000/paleo.721

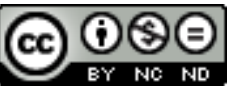

PALEO est mis à disposition selon les termes de la licence Creative Commons Attribution - Pas d'Utilisation Commerciale - Pas de Modification 4.0 International. 


\title{
LA GROTTE DES FÉES (Châtelperron, Allier) OU UNE INTERSTRATIFICATION «CHATELPERRONIEN-AURIGNACIEN» ILLUSOIRE. HISTOIRE DES FOUILLES, STRATIGRAPHIE ET DATATIONS
}

\author{
João ZILHAO (1), Francesco d'ERRICO (2), Jean-Guillaume BORDES (2), \\ Arnaud LENOBLE ${ }^{(3)}$, Jean-Pierre TEXIER ${ }^{(2)}$, Jean-Philippe RIGAUD ${ }^{(2)}$
}

\begin{abstract}
Résumé : Dans une publication récente, Gravina et collaborateurs (2005) admettent qu'une interstratification entre industries châtelperroniennes et aurignaciennes est avérée à la grotte des Fées, phénomène qui implique une longue contemporanéité entre les populations porteuses de ces deux technocomplexes. Cette interprétation, qui s'appuie essentiellement sur des datations numériques réalisées à partir d'échantillons provenant des fouilles de Delporte, a été vivement critiquée par Zilhao et al. (2006). Elle a cependant été maintenue par les premiers auteurs qui réfutent l'ensemble des arguments avancés par Zilhao et collaborateurs (Mellars et al. 2007)

Dans cet article, nous exposons en détail les informations et les arguments qui nous conduisent à rejeter l'idée d'une interstratification sur le site de la grotte des Fées. Les questions soulevées par Mellars et collaborateurs sont discutées. De nouveaux éléments sont également pris en compte. L'historique des travaux conduits sur le site depuis le XIX ${ }^{\ominus}$ siècle, les incohérences concernant la localisation des fouilles, différente selon les sources, la signification des datations ${ }^{14} \mathrm{C}$, l'analyse de l'industrie lithique et de la parure recueillie par H. Delporte montrent que ce site livre en fait un mélange de pièces châtelperroniennes et aurignaciennes sur l'ensemble de sa séquence et qu'il ne peut donc servir à étayer l'hypothèse d'une contemporanéité entre ces deux populations.
\end{abstract}

L'analyse de la faune associée aux industries met en évidence une accumulation par des carnivores. Sur cette base, les différentes possibilités d'association entre vestiges fauniques et lithiques sont discutées. La signification des dates ${ }^{14} \mathrm{C}$ est également évaluée à partir des différents modes de formation possibles du site.

L'interprétation la plus rigoureuse des données recueillies incite à penser que les dépôts fouillés par Delporte correspondent pour l'essentiel à des déblais de fouilles pratiquées au $\mathrm{XIX}^{\ominus}$ siècle. Seule une petite partie du site, en particulier à la base de la séquence, pourrait réellement correspondre à des témoins en place. Sur les treize dates obtenues par Mellars et collaborateurs (2007), dix ont été réalisées à partir de vestiges provenant de ce témoin. Elles suggèrent une occupation châtelperronienne autour de $39-40 \mathrm{ka}{ }^{14} \mathrm{C} \mathrm{BP}$ et une occupation par les seuls camivores dans l'intervalle $36,5 \mathrm{ka}-34,5 \mathrm{ka}{ }^{14} \mathrm{C}$ BP. La présence d'objets typiquement aurignaciens, au sein des déblais fouillés par Delporte, atteste d'une fréquentation sporadique du gisement par les porteurs de cette industrie. Ces vestiges aurignaciens sont susceptibles de provenir d'un niveau surmontant les dépôts moustériens et châtelperroniens.

Mots-clés : Transition Paléolithique moyen/Paléolithique supérieur, Grotte des Fées, Châtelperronien, Aurignacien, Interstratification, Stratigraphie, Datations.

Key-words: Middle to Upper Palaeolithic transition, Grotte des Fées, Chatelperronian, Aurignacian, Interstratification, Stratigraphy, Dating.

(1) Department of Archaeology and Anthropology, University of Bristol, 43 Woodland Road, Bristol BS8 1UU, UK joao.zilhao@bristol.ac.uk

(2) Université Bordeaux 1 - PACEA/UMR 5199 du CNRS, Institut de Préhistoire et de Géologie du Quatem a i re, UFR de Géologie, Bat. B18, Avenue des Facultés, 33405 Talence, France

- f.derrico@ipgq.u-bordkax1.fr - jg.bordes@ipgq.u-bordeaux1.fr - amaud.lenoble@ipgq.u-bordeaux1.fr

- jp.texier@ipgq.u-bordeaux1.fr - j.ph.rigaud@ipgq.u-bordeaux1.fr

(3) Département de Préhistoire - Muséum national d'Histoire Naturelle, UMR 5198 du CNRS, Musée de l'Homme, Palais de Chaillot, 17 Place du Trocadéro, 75116 Paris, France - amaud.lenoble@mnhn.fr 
THE GROTTE DES FÉES (CHÂTELPERRON, ALLIER), AN ILLUSORY "CHÂTELPERRONIAN-AURIGNACIAN" INTERSTRATIFICATION. EXCAVATION HISTORY, STRATIGRAPHY AND DATING.

The interstratification of Châtelperronian and Aurignacian layers at sites such as Le Piage, El Pendo and Roc de Combe have been for long the keystone of the Human Revolution paradigm, according to which cultural innovations of the Châtelperronian (omaments, decorated bone tools) must be interpreted as a byproduct of either "imitation without understanding" or "acculturation" of late Neanderthal communities. Over the last decade, however, the reappraisal of these sequences from a site formation perspective, coupled with critical analyses of the corpus of radiocarbon determinations for the two technocomplexes, have challenged this view, leading to suggestions that the emergence of the Châtelperronian predated any evidence for the Aurignacian or modern humans in Europe and, therefore, represented the Neanderthals' independent transition to cultural modemity.

More recently, based on a series of radiocarbon dates for the Grotte des Fées (Châtelperron, Allier, France), Gravina et al. (2005) argued that there was a genuinely in situ lens of Aurignacian material in the middle of that site's thick Châtelperronian sequence, implying Châtelperronian reoccupation after a brief Aurignacian incursion and, hence, the long-term regional coexistence of Neanderthals and modems, as predicted by "acculturation" or "imitation" models. Our subsequent analysis of the collections and associated documentation, kept at the Musée d'Archéologie Nationale (MAN), St.-Germain-en-Laye, France (Zilhão et al. 2006), led us to believe that the Châtelperronian-bearing deposits overlying that Aurignacian lens were 19th-century backfill, and to argue that interstratification was as illusory at the Grotte des Fées as everywhere else. Mellars et al. (2007), however, have since claimed refutation of our diagnosis and confirmation of Gravina et al.'s original conclusions.

Here, we challenge Mellars et al.'s claims by showing their interpretation to be in direct contradiction with the available empirical evidence. Our rebuttal is based on a new detailed examination of the historical and stratigraphic evidence, the radiocarbon determinations, and the provenience of the dated samples, as well as on a discussion of the faunal data, the ornaments and the taphonomy, technology, typology and refitting of the lithic assemblages.

At the Grotte des Fées, Delporte recognized five stratified Châtelperronian levels, characterized by a distinct reddish color (from top to bottom, B1, B2, B3, B4, B5) and lying above Mousterian deposits (level C). He also identified three intermediate sublevels (B3a, B4a, $\mathrm{B} 5 \mathrm{a})$ and noted a concentration of Aurignacian material in B4-B4a. The bone of contention resides in that both Gravina et al. (2005) and Mellars et al. (2007) fully accept the validity of this succession, while Zilhão et al. (2006) argued that: 1) levels B1-to-B3 were backfill; 2) only levels C and, to some extent, B4-to-B5, were conceivably intact, and 3) even if B4-to-B5 were broadly undisturbed, the best interpretation for the Aurignacian finds in B4-B4a would be post-depositional intrusion, not interstratification.

The Grotte des Fées had been extensively explored prior to Delporte's 1950s work and his excavations were neither recorded nor published with the detail needed for full testing of the different interpretations. Finds were not piece-plotted and there is no indication of from where exactly in the different areas excavated by Delporte the finds come.

Our analysis of the drawings and photographs illustrating the stratigraphy of the site suggests that Delporte's (1957) representation of the stratigraphy, upon which Gravina et al. and Mellars et al. base their argument, is a post facto schematic rendition, combining elements of the 1952 trench and the 1953-54 palier sud excavations, not the recording of any profile physically extant at any time during the excavation process. The unpublished photographs kept at the MAN alongside the artifact and faunal collections appear to be a more reliable source of information. Their evaluation suggests, considering the location of both Delporte's and previous, 19th-century excavation work, that at least the upper part of Delporte's profile cut through disturbed deposits and cannot be used to support the integrity of the sequence. In situ remnants may have been found in 1962 but, in the part of the site excavated at that time, Delporte failed to recognize the previously described interstratification, simply subdividing the deposits in two units, "B1-3" at the top, and "B4-5" at the bottom.

Analysis of the labeling and spatial origin of the dated bone samples identifies another pitfall into which Mellars et al. stumbled. The stratigraphic consistency of 10 out of the 13 radiocarbon dates available relates to the fact that those 10 dates were obtained on samples coming from the conceivably in situ remnants excavated in 1962. Three samples from level B5 date the Châtelperronian occupation to ca. 39-40 ka ${ }^{14} \mathrm{C}$ BP, while seven samples from level "B1-3" relate to carnivore denning at the site throughout the ca. 36.5$34.5 \mathrm{ka}{ }^{14} \mathrm{C}$ BP interval.

The lithics fit the expectation that the upper B1-to-B3 layers represent 19th-century backfill and cannot be interpreted as in situ Châtelperronian layers: the frequency of suface-weathered and broken pieces is much higher in those upper levels, wherein a postAurignacian foliate piece was also found, and there are major typological inconsistencies in the Aurignacian tools from all levels. This interpretation is contradicted neither by the few refittings found by Delporte and by us, nor by the stratigraphic position of the small number of typical Mousterian items recovered at the site.

Close analysis of the two personal ornaments collected by Delporte, a fox and a hind canine, does not support interstratification either. Both types of perforated teeth occur at Châtelperronian and Aurignacian sites. A preparation by scraping prior to punching the root, applied to the fox canine, is common in the Aurignacian but attested in the Châtelperronian as well. A stronger presumption of Aurignacian-ness is legitimate for the hind pendant but, even if Aurignacian, this object would still not support interstratification. It comes from B4, but the year of excavation is 1952, indicating that it comes from the backfill deposits across which Delporte excavated that year's longitudinal trench.

In short, our summary view of the stratigraphy of the Grotte des Fées is as follows: 
- we have no doubt that levels B1-to-B3 of Delporte's excavations are backfill, and we believe that some of the B4-to-B5 material - but in an unknown percentage, and clearly not all of it - comes from in situ remnant deposits;

- originally, the site contained a rich Châtelperronian fill capped by surficial deposits containing remains of later, sporadic human incursions, namely during the Aurignacian;

- the B5 samples may well date an in situ remnant preserved in the periphery of the Châtelperronian habitat excavated in the 19th century at the center of the chamber, while the "B1-3" samples clearly date a post-Châtelperronian camivore denning context located in a recess of the south wall of the cave, beyond the limits of the human occupation area.

Beyond its implications for the debate on Neanderthal-modemhuman contact and interaction, the Grotte des Fées controversy raises broader issues of methodology related to the more general archeological problem of how to deal with the evidence inherited from the fieldwork camied out by past generations of researchers. Gravina et al. and Mellars et al. simply went through a museum collection to obtain samples for radiocarbon dating, subsequently publishing the results with no critical analysis of excavation history and site formation process. Although several aspects of the evidence from the Grotte des Fées will no doubt remain unresolved, our analyses should at least have made it clear that, in such cases, much improved and more sophisticated research protocols have to be followed.

\section{1- INTRODUCTION}

Au cours des années cinquante et soixante, l'idée que les industries paléolithiques pourraient représenter de très longues traditions culturelles reflétant la coexistence, dans une même région, de groupes humains différents, s'est largement répandue parmi des préhistoriens. Elle est à l'origine du débat des années soixante-dix portant sur la variabilité fonctionnelle des industries dont l'expression la plus emblématique reste la controverse sur l'interprétation des faciès moustériens (Binford 1973 ; Bordes 1973). Mais, ce concept a également été appliquée au Paléolithique supérieur. II en est ainsi de l'hypothèse initialement défendue par Peyrony $(1933,1948)$, hypothèse selon laquelle la diversité des industries du début du Paléolithique supérieur exprime le développement de deux traditions techniques distinctes, l'une aurignacienne et l'autre " périgordienne ".

Par la suite, il est devenu évident que plusieurs millénaires séparaient le Périgordien inférieur, ou Châtelperronien, du Périgordien supérieur, ou Gravettien (Laville et al. 1980). De la même façon, il est apparu, à l'autre extrémité de ce long intenalle de 15000 ans de coexistence supposée, que l'Aurignacien V, placé au sommet de la séquence de Laugerie-Haute, ne saurait être considéré comme un Aurignacien vrai ou "typique" (Sonneville-Bordes 1982). $\mathrm{Si}$, en conséquence, l'idée d'une lignée périgordienne est tombée progressivement en désuétude, puis a finalement été abandonnée, cela s'est fait sans que soit remise en cause l'une de ses prémisses fondamentales, à savoir la contemporanéité entre Châtelperronien et Aurignacien, déduite des interstatifications constatées à Roc-deCombe par Bordes et Labrot (1967), au Piage par Champagne et Espitalié (1981) et sur le site d'EI Pendo, en Espagne, par Bernaldo de Quirós (1982). Aussi, la notion de groupes humains contemporains reste, au même titre que la fonction des sites ou que l'évolution intrinsèque des industries, une explication admise pour rendre compte de la variabilité des industries aux commencements du Paléolithique supérieur en Europe.

Jusqu'à la découverte du squelette de Saint-Césaire et de la reconnaissance de l'artisan des industries châtelperroniennes (Levêque et Vandermeersch 1980), il était largement admis que les technocomplexes du Paléolithique supérieur étaient l'œuvre de l'Homme anatomiquement modeme. Avec cette découverte, la signification paléoanthropologique de l'interstratification prit une toute autre toumure. Elle passa du statut de simple variabilité des productions de l'Homme moderne à celui d'un témoignage c rucial pour l'évaluation des comportements culturels qui caractérisent et distinguent les hommes modernes des " non-modernes ". Ainsi, admettant que les industries châtelpenoniennes et aurignaciennes sont respectivement l'expression des derniers néandertaliens et des premiers européens anatomiquement modernes, les interstratifications constatées dans les sites de Roc-de-Combe, du Piage, et d'El Pendo impliqueraient que des groupes 
humains distincts se sont maintenus durant des millénaires sur un même teritoire tout en gardant leur identité techno-culturelle, malgré de nombreuses opportunités de contact et d'échange. Dans le cadre du modèle de la " Human Revolution " (Mellars et Stringer 1989), les capacités cognitives, biologiques ou culturelles des Néanderthaliens sont moindres que celles des nouveaux arrivants anatomiquement modernes. L'interstratification est alors la clef de voûte d'une conception des innovations culturelles châtelperroniennes (parure, art mobilier) qui ne sont considérées que comme une "imitation sans compréhension ", ou comme le reflet d'une " acculturation " (Demars and Hublin 1989 ; Stringer et Gamble 1993 ; Hublin et al. 1996 ; Mellars 1999 ; Hublin 2000).

Au moment même où ces concepts étaient élaborés et diffusés, d'importants progrès concernant les processus de formation des sites et la taphonomie étaient réalisés. Or, les résultats obtenus dans ces domaines jetaient le doute sur la validité des observations supportant la notion d'interstratification (d'Errico et al. 1998 ; Rigaud 2001). Cette interrogation a débouché sur de nouvelles recherches, tant sur le terrain que dans les archives. Ces travaux ont ainsi permis une analyse critique des premières interprétations. Celles-ci ont montré que la séquence d'El Pendo est inversée et formée de dépôts en position secondaire (Montes et Sanguino 2001 ; Montes et al. 2005). De même, il est apparu que le secteur du Piage ayant livré l'interstratification est formé de dépôts de pente qui livrent, pêle-mêle, des vestiges aurignaciens et châtelperroniens ainsi que des pièces moustériennes fortement altérées, et que la lentille aurignacienne interstratifiée dans les couches châtelperroniennes du Roc-de-Combe n'est rien d'autre qu'une reconstruction théorique qui réinterprète des séries provenant de fouilles livrant un mélange de pièces gravettiennes, aurignaciennes, châtelperroniennes et moustériennes (Bordes 2002, 2003, 2006). A ceci, s'ajoute une prise de conscience des problèmes liés aux datations ${ }^{14} \mathrm{C}$ (difficultés de l'échantillonnage, contamination des objets datés...), notamment lorsqu'il s'agit d'ossements (Zilhão \& d'Errico 1999 ; Jöris et al. 2003 : Higham et al. 2006). Or, les révisions stratigraphiques menées en tenant compte de ces différents problèmes, ont établi l'antériorité chronologique du Châtelperronien vis-à-vis de l'Aurignacien. Ces résultats ont remis en cause les fondements de la théorie de la "Human Revolution ". Parce que son émergence est significativement antérieure à toute indice de présence aurignacienne ou d'Homme moderne en Europe, le Châtelperronien ne peut être interprété que comme l'expression d'une transition néanderthalienne indépendante vers la modernité culturelle (d'Errico et al. 1998 ; Zilhão 2001, 2006, 2007 ; d'Errico 2003).

La publication récente par Gravina et collaborateurs (2005) d'une série de dates ${ }^{14} \mathrm{C}$ de la séquence de la grotte des Fées (Châtelperron, Allier, France), site éponyme du Châtelperronien, a apporté de nouveaux éléments à ce débat. Dans cet article, les auteurs ont soutenu que la cohérence des dates démontrait que la lentille de vestiges aurignaciens, contenue au sein d'une épaisse série de dépôts châtelperroniens, était réelle- ment in situ. Ceci impliquait une réoccupation châtelperronienne du site à la suite d'un bref séjour par des Aurignaciens et, par voie de conséquence, une longue coexistence régionale entre hommes anatomiquement modernes et Néanderthaliens, telle que le prédit le modèle de l'acculturation. A l'inverse, notre analyse de la collection et des archives des fouilles Delporte déposées au Musée de Saint-Germain-en-Laye nous a conduits à conclure que les dépôts à industrie châtelperronienne surmontant cette lentille aurignacienne étaient des déblais du XIX ${ }^{\mathrm{e}}$ siècle. C'est pourquoi, nous avons soutenu que l'interstratification "ChâtelperronienAurignacien» de la grotte des Fées est illusoire, comme partout ailleurs (Zilhão et al. 2006). Cette dernière interprétation a été contestée par Mellars et collaborateurs (2007). Selon eux, la séquence archéologique de la grotte des Fées est bien en place et les conclusions du premier travail de Gravina et al. (2005) restent valides.

Mellars et collaborateurs (2007) ont essayé de réduire la divergence d'interprétation à un simple désaccord résultant d'une divergence d'approches. Leur démarche reposerait sur des faits avérés ; la nôtre, essentiellement théorique, serait fictive. Nous ne nions pas qu'une dimension théorique puisse expliquer pour partie notre lecture du site (tout comme celle de Mellars, d'ailleurs). Il est en outre évident que le statut final de cette interstratification a de nombreuses implications quant aux modèles de l'émergence de l'Homme moderne. Toutefois, le premier intérêt de cette controverse sur l'interprétation de l'archéoséquence de la grotte des Fées est de toucher à un problème méthodologique plus vaste : celui des fouilles anciennes et de l'utilisation de leurs résultats dans les débats archéologiques actuels. C'est pourquoi, nous nous sommes attachés à réunir une documentation suffisante, bien qu'imparfaite, pour alimenter une discussion sur l'histoire des fouilles ainsi que sur la taphonomie et la signification des séries recueillies. De plus, une mission de terrain sur le site a été réalisée de façon à disposer d'une meilleure estimation de la documentation de base.

Bien souvent, de vives controverses permettent une clarification de questions-clés. Le cas de la grotte des Fées ne fait pas exception à cette règle. L'exposé qui suit est une poursuite des précédents échanges et il aurait été difficile de ne pas le bâtir comme une réponse aux différentes questions soulevées par Mellars et ses collaborateurs. Aussi, pour chacune des questions abordées, nous rappelons d'abord le point de vue de nos détracteurs, sous la forme de citations complètes. Toute ambiguité est ainsi évitée. Puis nous exposons notre position en expliquant les raisons pour lesquelles notre point de vue ne peut s'accorder avec le leur. Après avoir traité la question de l'historique des fouilles, auquel nous apportons plusieurs éléments nouveaux, nous abordons successivement la stratigraphie, les datations et les séries de vestiges ellesmêmes. La discussion qui suit nous permet de proposer une synthèse sur le sujet. Les principales implications, tant méthodologiques que paléoanthropologiques, sont résumées en conclusion. 


\section{2- HISTORIQUE DES FOUILLES}

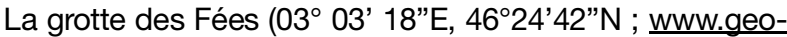
portail.fr) est, en fait, un ensemble de deux cavités, distinctes mais interconnectées. Toutes deux se trouvent de 5 à $6 \mathrm{~m}$ au-dessus du ruisseau, le Graveron. La première entrée est la grotte Poirrier et la seconde, la grotte Bailleau, ainsi nommées par Henri Delporte d'après le nom de ses prédécesseurs sur le site. A quelques mètres au nord de la grotte Bailleau, existe un troisième locus : la grotte effondrée. II s'agit, en fait, d'une chambre karstique dont la voûte a été exploitée en carrière au cours du XIX ${ }^{\mathrm{e}}$ siècle (Bailleau 1872 : 110, 118). C'est sur ce dernier site qu'Henri Delporte a repris les travaux de 1951 à 1954 (Delporte 1955, 1957, n.d. ; Delporte et al. 1999).

Comme le rapportent Bailleau $(1869,1872)$ et Delporte (1957), I'histoire du site débute dans les années 1840, par la découverte d'un riche gisement à faune quaternaire au cours de la construction de la ligne de chemin de fer reliant le bassin houiller de Bert-Montcombroux aux usines métallurgiques de Dompierre-sur-Besbre. De cette voie ferrée, il ne reste plus aujourd'hui qu'un chemin rural longeant le Graveron.

L'ingénieur en charge des travaux, A. Poirrier, était également paléontologue. C'est pourquoi il continua à exploiter les dépôts mis au jour lors des travaux de constru ction de la voie ferrée. De cette façon, il put constituer une importante collection paléontologique. Bailleau, un médecin local et préhistorien amateur, visita le site au cours des années 1864-1867. II fut alors frappé par le nombre de vestiges lithiques jonchant le sol. Alors que Poimer n'avait pas reconnu ou, tout du moins, avait ignoré ces objets archéologiques, leur présence conduisit Bailleau à entreprendre ses propres fouilles. Celles-ci débutèrent à l'automne 1867 et furent poursuivies jusque dans les années 1870-1872.

L'effort de Bailleau a d'abord porté sur deux locus. Le premier, la grotte Poirrier, contenait deux dépôts superposés : à la base, des sables et des limons déposés par le Graveron et au sommet, un niveau à céramique galloromaine. Aucun vestige faunique ou lithique pléistocène n'y a été recueilli. Ceci peut s'expliquer par le fait que l'entrée a été vidée, soit au cours de l'occupation gallo-romai ne, soit par Poirrier et les travaux de construction de la ligne de chemin de fer. Le second locus, la grotte Bailleau, était intact lorsque celui-ci entreprit ses travaux. Le sommet du remplissage était formé d'une terre noire accumulée récemment, probablement à partir d'un puits de jour, et dans laquelle Bailleau recueillit des ossements récents : Lapin, oiseaux et autres proies amenées par un renard. Sous ce premier niveau, il rencontra des fragments rocheux colmatés par des limons rougeâtres riches en restes de faune. De l'Hyène, de l'Ours des cavernes, du Cheval et du Renne y ont notamment été récoltés. Aucun objet archéologique n'a été recueilli, à l'exception de deux nodules dans lesquels Bailleau a reconnu des percuteurs utilisés pour briser les os, mais qui peuvent n'être qu'une composante naturelle du remplissage. La présence d'os fracturés et quelques exemplaires "d'outils en os " ont cependant conduit Bailleau à conclure à l'occupation du site par les hommes (Bailleau 1869 : 12 ; pl. I).

Ayant entamé le remplissage de la grotte sur une distance de $15 \mathrm{~m}$ depuis l'entrée et rencontrant de larges blocs ne permettant pas une exploration plus poussée de la galerie, Bailleau cessa ces travaux. II reporta alors son attention sur le troisième site, qu'il appela alors «le foyer», et dans lequel il trouva de très nombreux vestiges lithiques. Pour rendre compte de la différence de contenu, Bailleau avança, dans un premier temps, deux explications (Bailleau 1869 : 12-14). La première est d'ordre taphonomique : les zones les plus riches se seraient trouvées devant les grottes et auraient été détruites lors de la construction de la voie ferrée. La seconde est d'ordre fonctionnel : les habitants des deux grottes auraient aménagé la zone du «foyer» pour regrouper les activités de traitement des ressources alimentaires et pour la confection de leur outillage. Au cours de l'année 1870, il réalisa toutefois que ce «foyer» comespondait en fait à la zone d'entrée d'une troisième cavité dont la voûte était effondrée. II comprit également que les blocs, dont une grande partie avait été extraite au cours des travaux d'exploitation, étaient le résultat de ce démantèlement et que le sédiment qui constituait la surface du sol était le sommet d'un remplissage de grotte. A la suite de ce constat, il changea d'opinion et considéra que seul ce troisième site avait été occupé par les hommes préhistoriques (Bailleau 1872 : 112, 116-118). Dans son demier article sur la grotte des fées, en date du 20 mai 1872, les conclusions de Bailleau laissent entendre que ses travaux sur le site sont achevés (Bailleau 1872: 126) ${ }^{4}$.

Jusqu'en 1951, date à laquelle Delporte entreprit une série de sondages, aucune fouille n'a été réalisée sur le site. La seule contribution notable fut celle de Lacaille (1947) qui publia les collections du XIX siècle, conservées en Angleterre. Les sondages de Delporte se sont avérés négatifs dans les grottes Poirrier et Bailleau. En revanche, cet auteur annonça alors avoir retrouvé un témoin préservé des fouilles anciennes au sein de la grotte effondrée. Cette découverte donna lieu à une série de fouilles au cours desquelles plusieurs plans et profils topographiques ont été élaborés. Ces documents sont reproduits dans les figures 1 et 2 .

Cette documentation est annotée dans la figure 3 , de façon à produire une vue synthétique de l'historique des fouilles. À ce propos, il faut noter une divergence de détail dans les écrits de Delporte quant à la date des premiers travaux réalisés dans le palier nord : 1952 (Delporte 1955 : 81) ou 1953 (Delporte n.d. : 10 ; dans ce rapport, la date de 1952 n'est mentionnée qu'à propos de la fouille de la tranchée longitudinale initiale). Dans la mesure où cette seconde référence fait sens et qu'elle est la dernière en date, nous l'avons retenue pour l'élaboration de la figure 3 .

(4) "Avis aux chercheurs : de mon côté, si le hasard vient seconder mes espérances, je serais heureux de recommencer de nouvelles fouilles et d'en faire l'objet de nouvelles communications " 
(a)

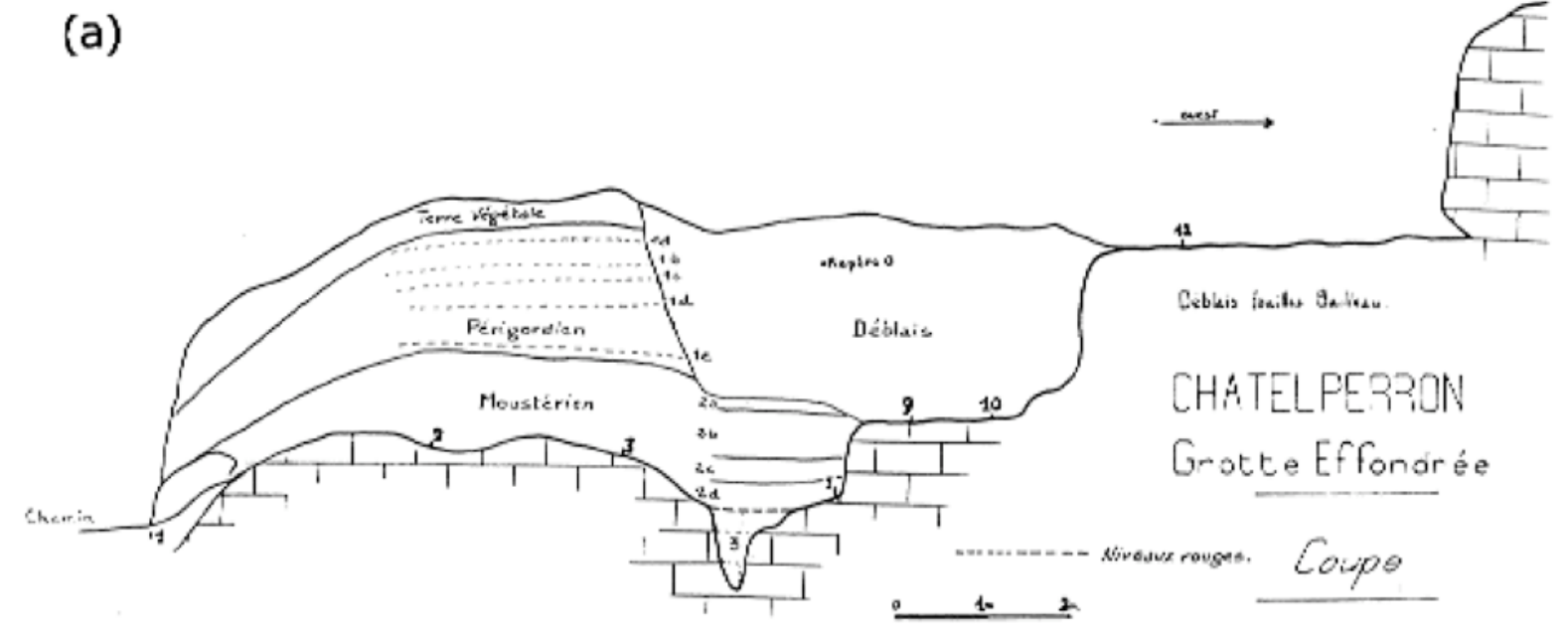

Figure 1 - Documents topographiques de la grotte effondrée publiés par H. Delporte.

(a) profil longitudinal relevé à la suite de la campagne 1954 (Delporte 1955: fig. 2).

(b) plan produit à l'issue de la même campagne de fouille (Delporte 1955 : fig. 1, 1957: PI. I).

Figure 1 - Delporte's published topographic documentation).

(a) Longitudinal profile after the 1954 season (Delporte 1955: Fig. 2).

(b) Plan after the 1954 season

(b)

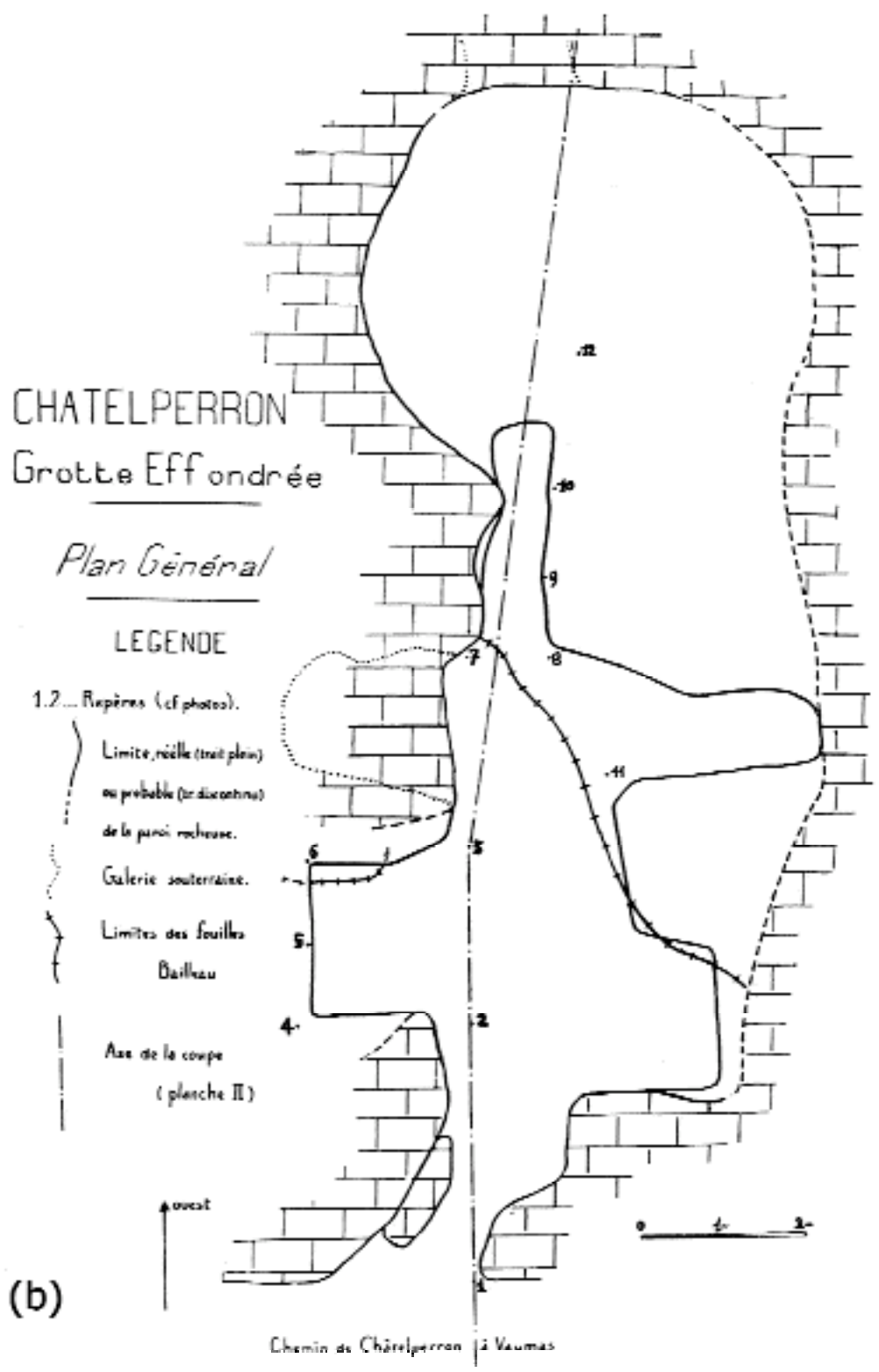



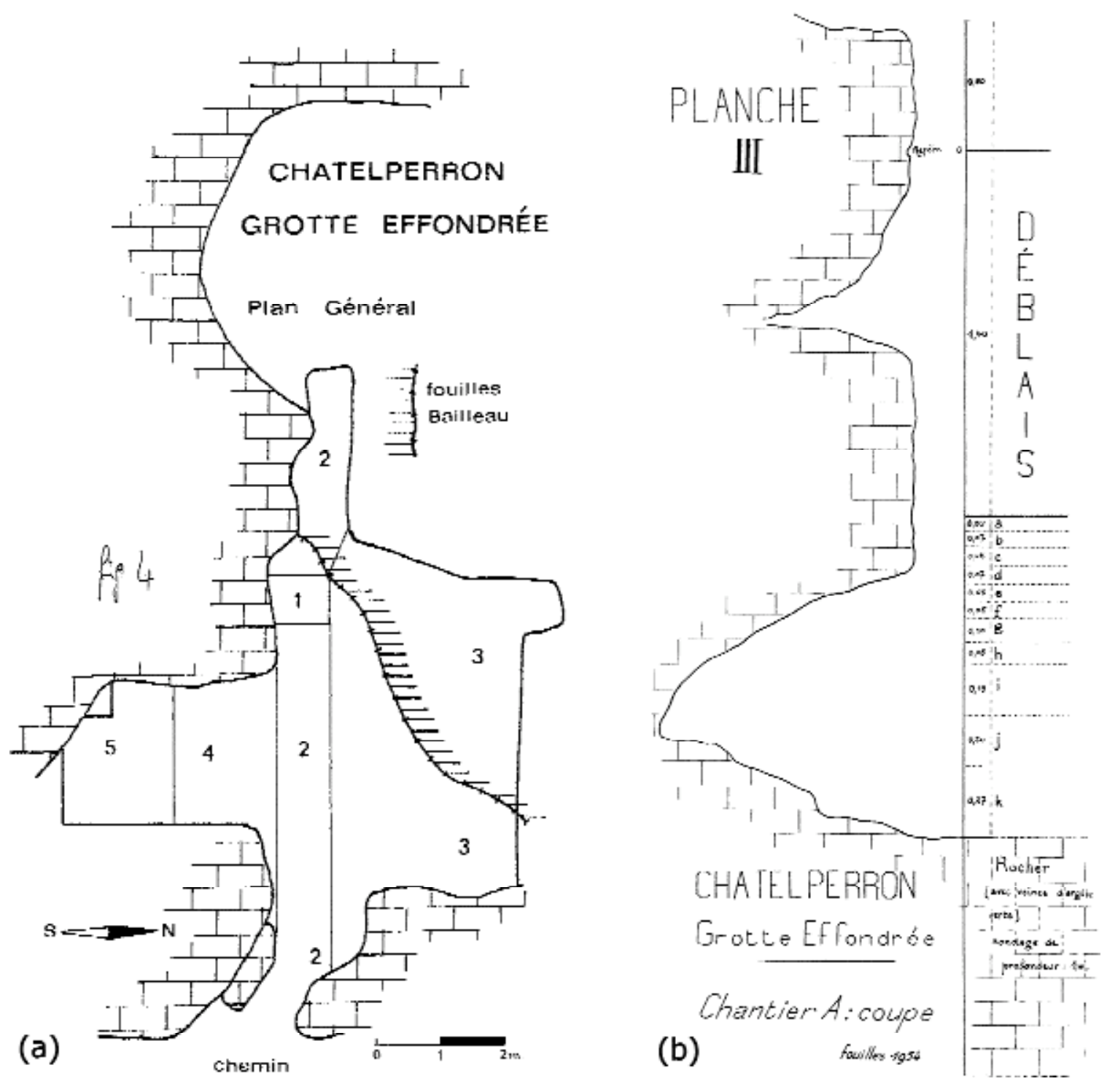

Figure 2 - Archive inédite des fouilles Delporte (a) la fig. 1, l'annotation "fouilles Bailleau" correspond aux légendes "déblais" et "déblais fouilles Bailleau" du profil. Sur ce plan, les secteurs sont numérotés comme suit : 1. sondage initial de 1951 ; 2-est et 2-centreindiquent la tranchée de 1952 ; 2-ouest et 3-ouest correspondent aux extensions de 1953-1954 au sein des déblais accumulés contre les murs nord et ouest de la salle ; 3-est est le pallier nord fouillé en 1952 et 1954 ; le secteur 4 est le palier sud fouillé en 1953-1954; le secteur 5, qui n'est pas représenté dans le plan de 1954, ne peut donc correspondre qu'au locus fouillé en 1962. (b) profil transversal au niveau de la galerie inférieure comblée de dépôts moustériens (Delporte n.d.: Planche III), situé entre les repères 3 et 7 du profil longitudinal de la fig. 1 (a).

Figure 2 - Delporte's unpublished excavation documents. (a) Last excavation plan (n.d.: Figure 4), where, by comparison with figure 1, the "fouilles Bailleau" caption corresponds here to the profile's "déblais" and "déblais fouilles Bailleau" (i.e., to backfill), and where the different numbered areas are as follows: area 1 is the initial 1951 test pit; 2-east and 2-middle mark the 1952 t rench; 2-west and 3-west mark the 1953-1954 perpendicular extensions of this trench into the backfill accumulated against the northemand western walls of the cave; 3-east is the palier nord excavated in 1952 and 1954; area 4 is the palier sud excavated in 1953-54; area 5, not represented in the 1954 plan, can only correspond, therefore, to the area excavated in 1962. (b) Transversal profile in the area (between points 3 and 7 of the longitudinal profile in fig. 1 (a) of the inferior gallery with a Mousterian fill (Delporte n.d.: PI. III). 

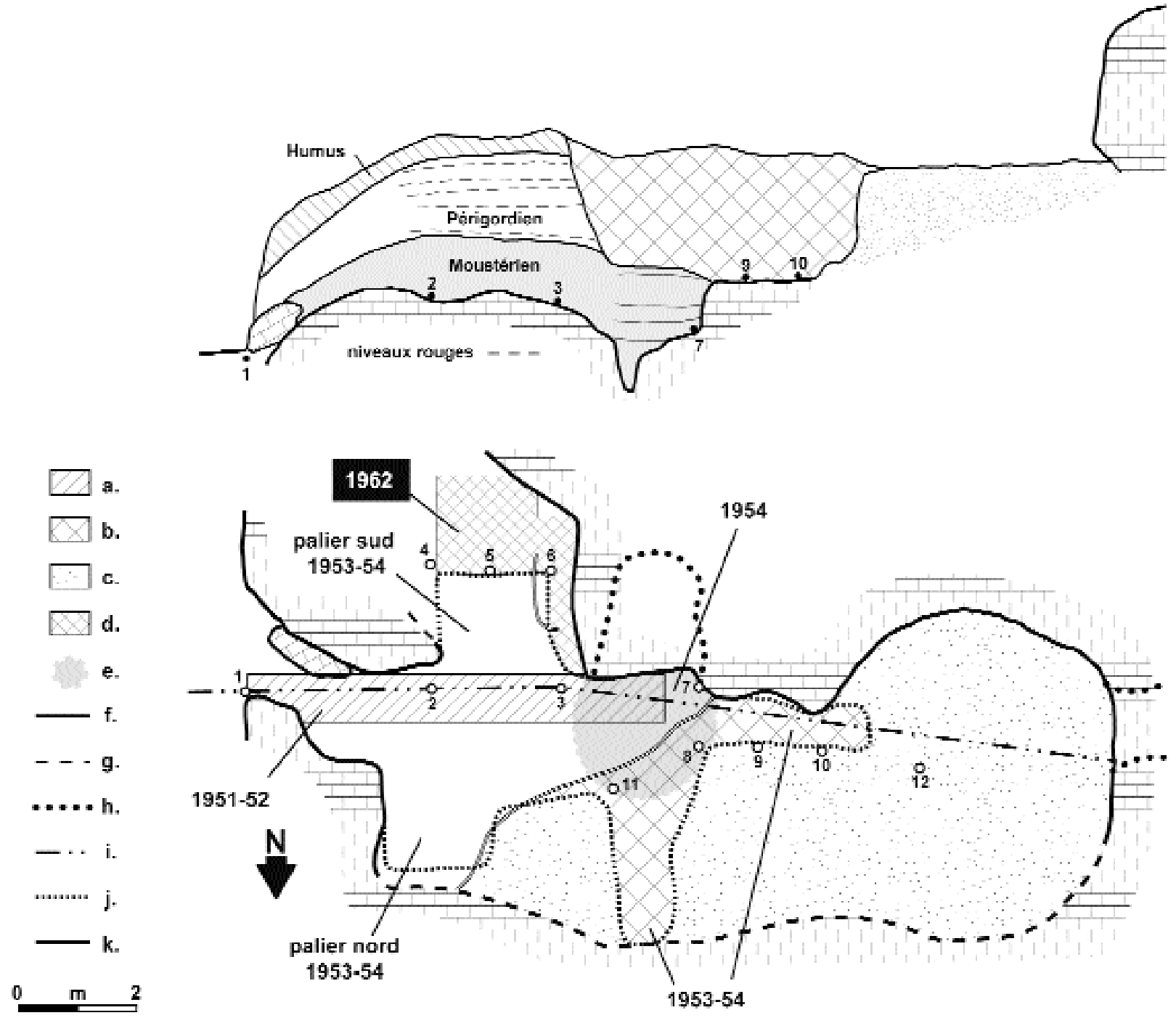

Figure 3 - Historique de la fouille de la grotte des Fées (modifié d'après Delporte 1955, 1957, n.d. ; les points numérotés sont les repères topographiques). En haut, profil longitudinal établi le long de la tranchée est-ouest de 1952. Des repères topographiques 2 à 3, la stratigraphie relevée par Delporte est identique à celle illustrée schématiquement pour le palier sud fouillé ultérieurement (cf. fig. 4). En bas, plan de la cavité avec localisation des secteurs fouillés par Delporte : $a$. tranchée initiale ; $b$. tranchée creusée dans le déblai Bailleau ; c. déblai Bailleau couvrant le rocher ; d. secteur fouillé en 1962 ; e. niveaux moustériens in situ observés dans un niveau inférieur de galerie ; $f$. paroi observée de la cavité ; g. prolongement probable de la paroi ; h. parois du niveau inférieur de galerie ; $i$. axe du profil longitudinal ; j. limites des fouilles Delporte de 1954 ; $k$. limite des fouilles Bailleau à la profondeur des niveaux moustériens.

Figure 3 - Grotte des Fées excavation history (reconstructed after Delporte, 1955, 1957, n.d., the numbered points are topographic markers). Above, longitudinal profile along the E-W trench of 1952; between points 2 and 3, the stratigraphy recorded here by Delporte is identical to that schematically illustrated in the subsequently excavated palier sud (cf. Fig. 4). Below, plan of the cave with indication of the areas excavated by Delporte: $a$. initial test and trench; $b$. trenches into Bailleau's backfill; $c$. Bailleau's backfill over bedrock; $d$. areas excavated in 1962; e. in situ Mousterian levels in an inferior gallery; $f$. observed limit of the cave wall; $g$. probable limit of the cave wall; $h$. walls of underground galleries; $i$. axis of the longitudinal profile; $j$. limits of Delporte's 1954 excavations; $k$. limits of Bailleau's excavations at the elevation of the Mousterian. 
L'élaboration de cette figure 3 se base sur l'idée que les travaux réalisés par Delporte en 1962, les seuls dont aucune publication ne rend compte, ont concerné le palier sud. Ce dernier aurait alors été étendu de deux mètres au-delà des coupes existantes. En même temps, le témoin ouest de 1954 aurait été fouillé jusqu'à atteindre la paroi de la cavité. Aucune légende du plan de 1962, reproduit dans la figure 2(a) et issu du manuscrit incomplet de Delporte (n.d.), conservé au Musée d'Archéologie Nationale, n'a été retrouvée. C'est pour- quoi notre argumentation repose sur les éléments suivants (Delporte op. cit.) :

"Nos travaux ont été organisés en trois phases successives (fig. 4):

- sondage à proximité de la paroi (1951), sondage qui nous a révélé l'existence de couches vierges,

- tranchée longitudinale (1952), qui nous a permis de préciser l'importance et la localisation des couches vierges,

- décapage par couches des paliers situés de part et d'autre de la tranchée $(1953,1954,1962)$."

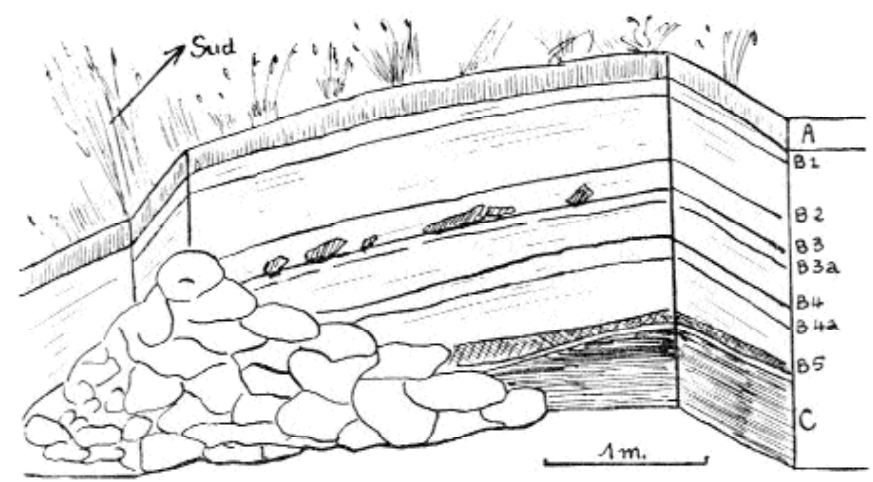

\begin{tabular}{|c|c|c|c|}
\hline$\stackrel{Ð}{5}$ & $1951-53$ & 1954-55 & 1962 \\
\hline 흐 & $1 a$ & B1 & \\
\hline 㫕 & $1 \mathrm{~b}$ & B2 & R1-3 \\
\hline @ & $1 \mathrm{c}$ & B3 & $81-2$ \\
\hline 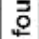 & $1 c^{\prime}$ & B3a & \\
\hline $\mathscr{d}$ & 1d & B4 & \\
\hline$x$ & 1d' & B4a & \\
\hline 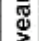 & $1 \mathrm{e}$ & B5 & 47 \\
\hline$\geq$ & $1 \mathrm{e}^{\prime}$ & B5a & \\
\hline
\end{tabular}

Stratigraphie du palier Sud (repères 4-5-6 du plan général).
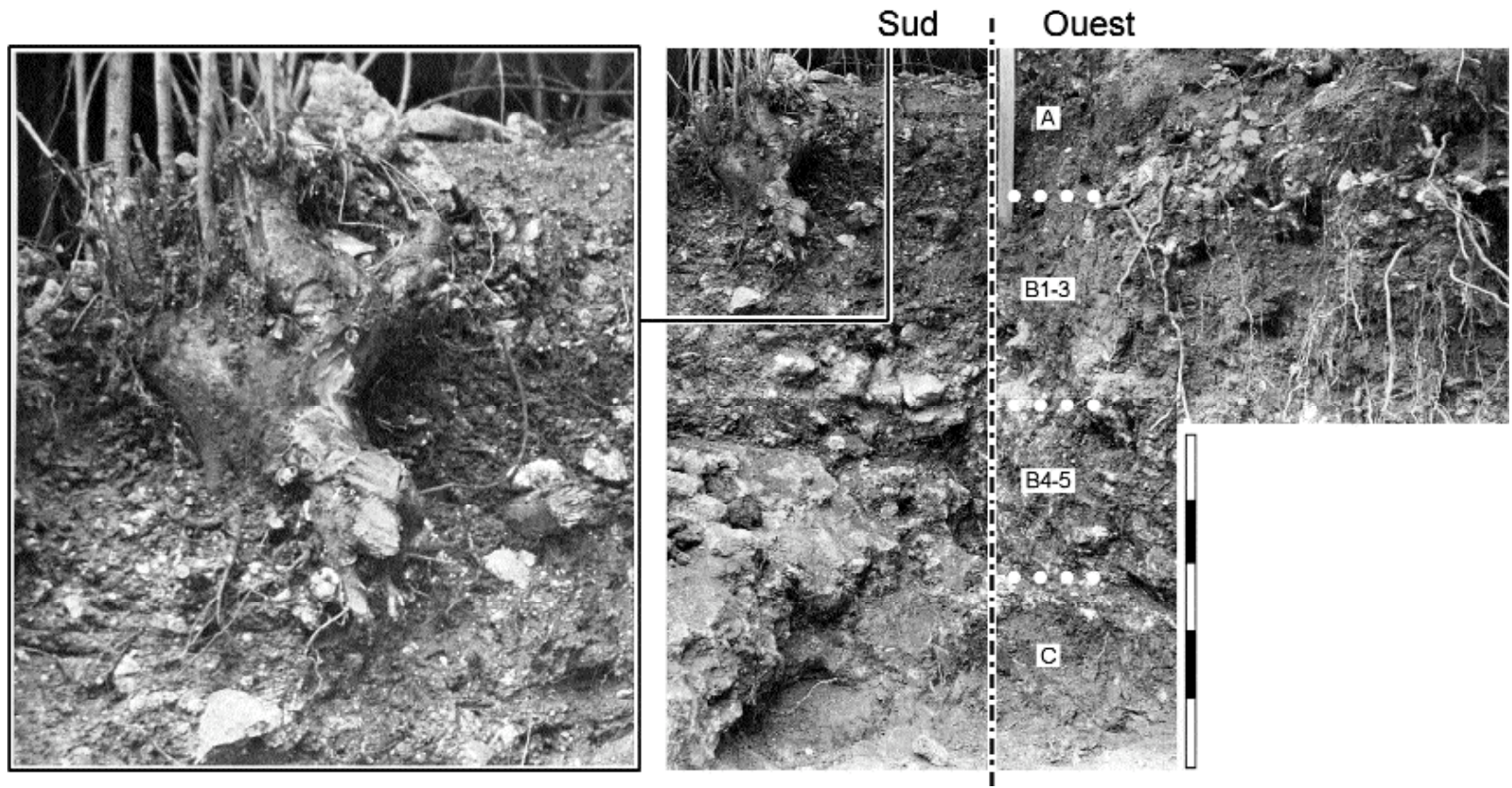

Figure 4 - En haut, à gauche : stratigraphie schématique du palier sud (Delporte 1957). En haut, à droite : tableau d'équivalence des différentes dénominations stratigraphiques utilisées par Delporte lors des différentes campagnes de fouilles. En bas : vue du profil stratigraphique de la grotte des Fées par composition photographique à partir des différents clichés pris par Delporte, probablement le coin sud-ouest du palier sud (échelle : $1 \mathrm{~m}$ ) et agrandissement de l'encart de la partie sud du profil (selon Zilhão et al. 2006: fig. 1).

Figure 4 - Top left: schematic stratigraphy of the south level (Delporte, 1957). Top right: equivalence between the different level designations used by Delporte over the years. Bottom: mosaic of Delporte's photos of profiles from the Grotte des Fées, probably documenting the SW corner of the palier sud (scale bar: $1 \mathrm{~m}$ ), and blown-up view of the framed area in the southern face (after Zilhão et al. 2006: fig. 1). 
Delporte n'a pas donné d'explication aux huit ans d'interruption des travaux à la grotte des Fées, de 1954 à 1962. On ne sait pas non plus pourquoi la monographie (Delporte n.d.) est restée inachevée. Ses derniers écrits sur la grotte des Fées correspondent à un petit ouvrage de vulgarisation (Delporte et al. 1999). Les différentes parties de ce travail sont signées indépendamment et la contribution de Delporte ne concerne que l'historique des fouilles et des collections, la biographie de Bailleau et l'orthographe exacte des cultures nommées d'après le site. La description de la stratigraphie et des objets recueillis au cours de ses fouilles est faite par F. Surmely et A. Urgal, Delporte ne faisant que cosigner deux paragraphes sur la signification des objets aurignaciens trouvés dans le site. Dans cette demière partie, la grotte des Fées est présentée à la lumière de l'interprétation de Bordes sur les interstratifications du Piage et du Roc-de-Combe. II considère donc celles de la grotte des Fées comme le résultat d'une utilisation successive du site par différents groupes humains. On doit cependant remarquer que cette interprétation diffère de celles soutenues auparavant par Delporte (e. g. 1957 : 474). Selon ces premières vues, les quelques pièces aurignaciennes trouvées dans un ensemble châtelperronien homogène ne représentent pas un horizon d'occupation distinct, mais une influence culturelle extra-régionale. Dans ce scénario, les Châtelperroniens du site éponyme, situé au nord du Massif central, auraient appartenu à une phase tardive de cette culture, contemporaine de l'Aurignacien du Bassin aquitain. Ce sont alors les contacts entre les deux groupes qui expliquent l'abandon, par les Châtelperroniens, d'objets aux affinités aurignaciennes qui auraient été acquis par échange ou fabriqués à partir de concepts techniques empruntés.

L'effort tardif de Delporte et al. (1999) pour intégrer le site de Châtelperron dans la vision orthodoxe d'une interstratification contraste avec le fait que Bordes lui-même ne s'est jamais appuyé sur la grotte effondrée pour défendre l'opinion d'un développement parallèle des groupes aurignaciens et périgordiens. Dans les discussions consacrées au sujet, Bordes ignore ce site, à l'exception d'une mention dans une liste de vieilles fouilles ayant livré des séries de "Périgordien ancien" (Bordes 1968 : 60). Sur cette question du Châtelperronien et de ses relations avec l'Aurignacien, les nouvelles générations de chercheurs ont gardé la même attitude. Ainsi, Harrold (1988) ne mentionne la grotte des Fées qu'une seule fois, dans un tableau où le site est inclus parmi ceux ayant livré des exemples de parures en contexte châtelperronien. De la même façon, Pelegrin (1995) confirme la nature aurignacienne des vestiges rencontrés par Delporte au sein de sa séquence châ- telpenonienne mais ne se base pas sur ce diagnostic pour appuyer le modèle de l'interstratification.

Le fait que plus de cinquante ans après, tous les chercheurs travaillant sur le Châtelperronien ignorent complètement le site de la grotte des Fées dans leur discussion sur l'interstratification, reflète le scepticisme de la communauté des paléolithiciens français sur la fiabilité des données provenant de ce site. Rétrospectivement, ce doute était tout à fait justifié, ce qui rend d'autant plus surprenant la confiance que Mellars et al. (2007 : 3662) placent dans ce gisement pour prétendre qu'un «flux et un reflux" de populations châtelperroniennes et aurignaciennes sont " fortement étayés par les données archéologiques " de la séquence de la grotte des Fées. En fait, la thèse que nous développons dans cet article est à l'exact opposé de cette dernière interprétation.

\section{3 - STRATIGRAPHIE}

Dans le secteur intact du site, au-dessus des niveaux moustériens (couche C) et sous l'humus (couche A), Delporte a reconnu une stratigraphie formée de cinq niveaux châtelperroniens. Ces niveaux se caractérisent par leur coloration rouge et ont été dénommés, de haut en bas, B1, B2, B3, B4 et $\mathrm{B} 5$. Trois horizons intermédiaires ont également été distingués (B B3a, B4a, B5a) ainsi qu'une concentration de vestiges aurignaciens qui a été observée au sein des couches B4 et B4a ${ }^{5}$. Gravina et alii (2005), et, à leur suite, Mellars et alii (2007) ont accepté sans réserve cette stratigraphie. En revanche, pour notre part, nous avons développé des arguments pour montrer que les niveaux B1 à B3 représentent en fait des déblais et que seuls les niveaux $\mathrm{C}, \mathrm{B} 5$ et $\mathrm{B} 4$ peuvent raisonnablement être considérés comme non remaniés. Dans le cadre de cette hypothèse, la meilleure façon d'expliquer la présence des pièces aurignaciennes au sein des niveaux $B 4 a$ et B4 est d'invoquer une intrusion post-dépositionelle et non pas une interstratification (Zilhão et al. 2006).

\section{1 - Dessin et photos}

Notre argumentation est notamment basée sur des documents photographiques retrouvés au sein des archives «Delporte» conservées au MAN (fig. 4). Mellars et al. ont reconnu, dans cette série de clichés, le coin sud-ouest du palier sud, pour lequel un relevé stratigraphique est également disponible (Delporte 1957: fig. 2). Ce faisant, ils ont admis que la section ouest du profil était perturbée et ont expliqué le désaccord qui en résulte (le dessin de la stratigraphie ne laisse apparaître aucune perturbation) par le fait que les vues auraient été prises à un moment différent de la fouille :

(5) Dans son compte-rendu au Congrès Préhistorique de France en juillet 1956, Delporte indique que les travaux ont débuté en 1951 (p. 455) et qu'ils se sont poursuivis durant quatre ans (Delporte 1957 : 455 ; p. 469-470). Ses autres compte-rendus (Delporte 1955, 1964) sont cohérents avec cette information puisqu'ils font mention de fouilles au cours des années 1951, 1952, 1953, 1954 et 1962. En conséquence, nous supposons que les références à des travaux réalisés en 1955 et 1956 (cf. Gravina et al., 2005 : 51) sont des erreurs de marquage, ou de lecture, et nous avons modifié notre table d'équivalence entre les différentes dénominations stratigraphiques selon Zilhao et al., 2006 (fig. 1 ; cf. fig. 4 du présent article). II faut noter que, dans son rapport de fouille de 1954, Delporte (1955) a désigné les différentes unités stratigraphiques avec des chiffres : 0,1,2, soit les équivalents de la nomenclature $A-B-C$ retenue dans Delporte (1957). Cette dernière a été probablement établie postérieurement à la fouille comme en témoigne les très fréquents doubles marquages observés au sein de la collection lithique. 
“Delporte a noté que la terminaison orientale [souligné par nos soins] des fouilles du XIXe siècle se surimposait, sur une courte distance $(\sim 30 \mathrm{~cm}$.), à l'extrémité ouest de la section dessinée (...) le dépôt représenté par Delporte à l'angle ouest de son relevé, qui se caractérise par une masse dense de racines protubérantes, représente donc les déblais non consolidés des fouilles anciennes, tandis que la section illustrée sur la coupe méridionale adjacente (partiellement photographiée à la gauche du cliché et dans lequel aucune racine sortant de la coupe ne peut être vue) représente les niveaux d'occupation in situ (...). Ceci, bien sûr, implique vraisemblablement que le dessin des coupes de la tranchée a été réalisée par Delporte avant que les fouilles ne soient étendues aux dépens de la zone présentant le recouvrement avec les déblais des fouilles anciennes (...)" (p. 3659). ${ }^{6}$

Les écrits de Delporte indiquent que la fouille du palier sud a été réalisée en deux ans (1953-1954). Il est donc possible que le profil longitudinal corresponde à un état de fouille antérieur à celui rapporté à la fin de la campagne de 1954 (cf. fig. 1, 3). Toutefois, dans leur raisonnement, Mellars et ses collègues font une erreur de taille, à savoir confondre la limite d'extension en direction de l'est avec ce qui est la bordure en direction de l'ouest des fouilles du XIX ${ }^{\circ}$ siècle. Les écrits de Delporte (1957 : 456) ne laissent place à aucun doute quant au fait que la ligne figurée sur son plan désignée en tant que "limites des fouilles Bailleau" (cf. fig. 1 , 3) ne peut être interprétée que comme la limite d'extension d'une fouille située entre cette ligne et l'entrée de la cavité. Sa description de la localisation des paliers nord et sud vis-à-vis des fouilles Bailleau est très claire : Delporte a travaillé au nord et au sud, et non pas à l'est de la zone fouillée au $\mathrm{XIX}^{\mathrm{e}}$ siècle $^{6}$. La ligne figurée pour situer la limite des fouilles Bailleau ne peut donc représenter, dans le palier sud, que la limite d'extension des anciennes fouilles en direction de l'ouest et, dans le palier nord et dans la partie centrale du gisement, la limite septentrionale de ces mêmes fouilles. Dans ces circonstances, tout profil relevé au niveau du palier sud, avant que la fouille ait atteint la zone de recouvrement, aurait recoupé des dépôts remaniés. Ce n'est qu'après avoir atteint cette zone de contact que Delporte a pu retrouver des dépôts anciens (fig. 5).

Cette erreur topographique peut être rectifiée. Mais une telle correction implique de reconsidérer l'ordre d'établissement des documents. En effet, il faudrait alors admettre que le dessin de coupe représente des dépôts in situ mis au jour au-delà des limites de la fouille Bailleau, tandis que la photographie illustre des coupes faites au sein des déblais au cours de la fouille. Dans ce cas, l'explication proposée par Mellars et alii tient-elle encore ? C'est possible, bien qu'il reste à préciser la localisation du levé stra- tigraphique par rapport aux indications données par Delporte sur son plan (fig. 6). Sur ce levé, la partie ouest de la section dessinée est large d'un mètre, tandis qu'elle aurait du être supérieure à deux mètres si elle se plaçait au-delà des limites de la fouille Bailleau. De la même façon, la partie sud de la section relevée est large de plus de trois mètres, tandis qu'à la fin de la campagne 1954, le palier sud atteignait au plus deux mètres de largeur et, ce, quelle que soit la section considérée. Par ailleurs, tout profil de trois mètres de large et encaissé d'un mètre par rapport à la tranchée de 1952 devrait faire apparaitre les parois de la cavité aux deux extrémités du levé, et non pas un témoin du remplissage. Qui plus est, si elle reproduit des dépôts non perturbés au-delà de la limite des fouilles Bailleau, la partie sud de la coupe levée peut difficilement livrer une séquence parfaitement identique à celle décrite pour la section sud de la tranchée de 1952 (cf. fig. 1,3). Ces incohérences montrent, comme nous l'avons déjà écrit, que le dessin publié par Delporte en 1957 (fig. 4) est, non pas le levé d'une coupe qui a réellement existé, mais plutôt une représentation schématique de l'interprétation stratigraphique du site, établie postérieurement à la fouille, et réalisée en combinant des éléments observés sur la tranchée de 1952 à des observations faites au cours de la fouille du palier sud. En conséquence, et contrairement à l'opinion de Mellars et alii, il est clair que les photographies sont des documents beaucoup plus fiables pour témoigner de la nature des dépôts que ne l'est le dessin. En effet, celui-ci n'est pas un enregistrement objectif d'une réalité de terrain, mais seulement l'expression graphique de la stratigraphie synthétique du site telle que l'a perçue le fouilleur.

\section{2 - Témoins in situ dans la section méridionale du} palier sud?

Les données topographiques, révisées à la lumière des documents photographiques, suffisent à démontrer que les dépôts fouillés par Delporte sur la bordure ouest du palier sud ne sont que des déblais. En revanche, Delporte a pu rencontrer des niveaux en place plus à l'est. Dans ce cas, ces niveaux devraient apparaître sur la section sud du palier et c'est, en effet, ce que soutiennent Mellars et alii. Or, ce que montrent les photographies est différent : la souche d'arbre, figurée dans l'encart de la figure 4, présente un diamètre d'au mois $40 \mathrm{~cm}$ et pénètre le dépôt sur une profondeur minimale de $60 \mathrm{~cm}$. Etant admis que la couche sommitale A n'est épaisse que de $30 \mathrm{~cm}$ (Delporte 1955: 81), cette souche s'enracine bien au-delà de l'humus et les racines qui la prolongent pénètrent les niveaux $\mathrm{B} 1$ à $\mathrm{B} 3$. Aussi, et avec le même argument que Mellars et alii, les photos montrent que la section sud est également perturbée et $\mathrm{ce}$, de façon bien plus importante que ne le suggèrent les

(6) "Delporte recorded that the eastern limit of the 19th-century excavations overlapped for a short distance (ca. $30 \mathrm{~cm}$ ) into the western end of this box section. (...) the deposits eventually exposed by Delporte in the western face of this section, marked by a dense mass of protruding roots, does [sic] indeed represent the loose backdirt of the earlier excavations, whereas the section exposed in the immediately adjacent southern face (partially shown on the left-hand side of the photograph, and with no visible protruding roots) represents the in situ occupation levels (...). This of course would presumably imply that the drawn section of this trench was recorded by Delporte before the extension of the excavation into the overlap zone with the 19thcenturyexcavations (...)" (Mellars et al. 2007: 3659). 

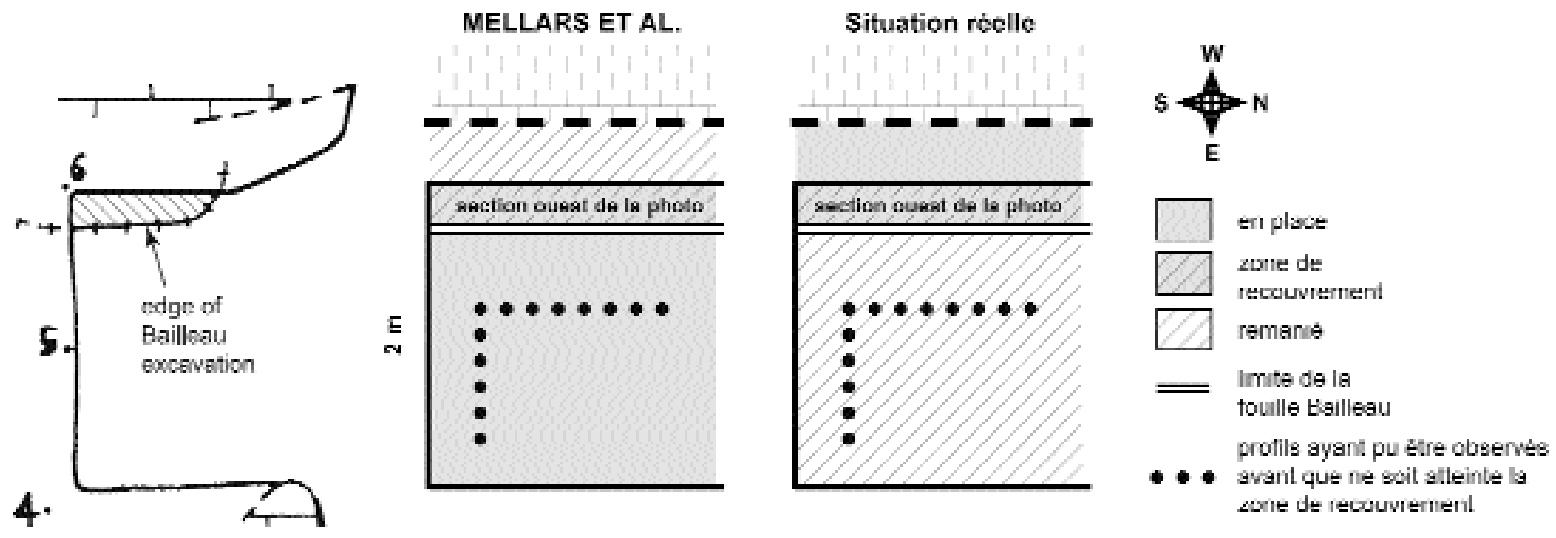

Figure 5 - Détail du plan du palier sud selon Mellars et al. (2007 : fig. 1) et interprétation alternative de la signification de la limite des fouilles Bailleau indiquée par Delporte dans son plan. Dans la mesure où la ligne dessinée par Delporte représente la limite ouest de l'extension des fouilles anciennes, et non pas la limite est, tout profil dessiné à l'intérieur du palier sud, avant la zone de recouvrement, devra faire apparaître des dépôts perturbés, de la base au sommet.

Figure 5 - Blown-up plan of the palier sud as published by Mellars et al. (2007: Fig. 1) and why their topological model for the discrepancy between intact deposits in the drawing and disturbed deposits in the photos is erroneous. Since Delporte's line represents the western, not the eastern limit of the 19th-century excavations, any profiles drawn inside the palier sud before the overlap zone was reached would have cut through disturbed deposits from top to bottom.

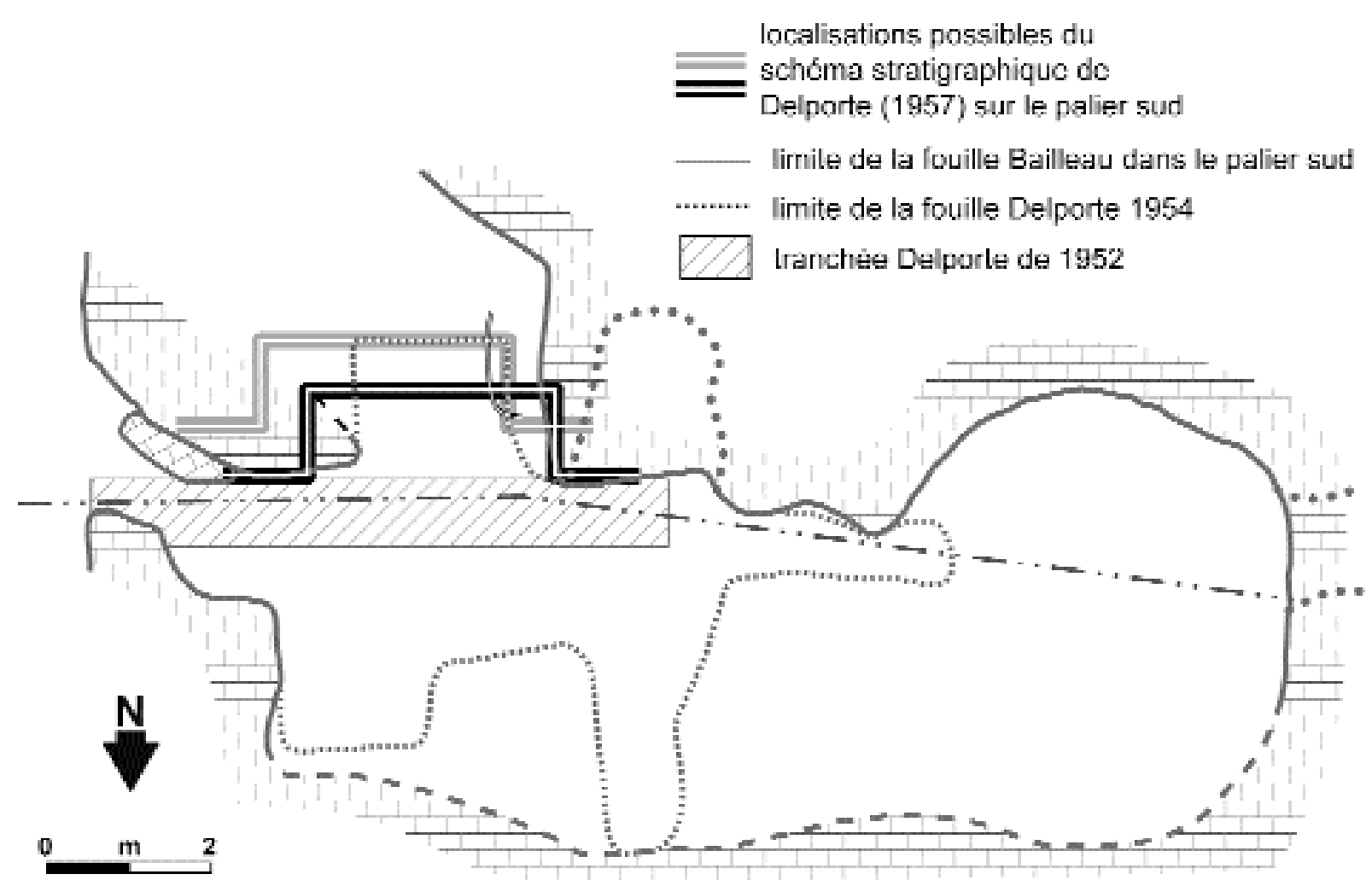

Figure 6 - Le schéma stratigraphique (Delporte 1957) et le plan de fouille (Delporte 1955) représentent la situation du site après la campagne de fouille de 1954. L'incohérence entre ces deux documents indique que le dessin est une réalisation postérieure à la fouille et non pas une représentation exacte du profil observé.

Figure 6 - The spatial inconsistency between the stratigraphic drawing of the palier sud in Delporte (1957) and the plan of the excavations in Delporte (1955), both recording the situation at the site after the 1954 field season, makes it clear that the drawing was meant to be a post facto graphic rendition of the excavator's stratigraphic model, not the accurate representation of observed profiles. 
fines racines, pourtant évidentes, qui sortent de la section ouest. Ce constat n'est d'ailleurs pas une surprise lorsqu'on considère la localisation des limites des fouilles Bailleau ou leur morphologie. En effet, d'après Delporte $(1955$ : 79,81) : “ la limite des fouilles Bailleau n'est qu'approximative : elle a en effet été relevée au niveau de la couche moustérienne et elle tend à s'évaser dans les niveaux périgordiens ". A hauteur des niveaux châtelperroniens, du fait de cet évasement, les limites des fouilles Bailleau doivent donc se situer bien au-delà de la bordure méridionale du palier sud dégagé par Delporte en 1954.

Plus encore, la géométrie de la fouille Bailleau implique que la zone de recouvrement, notée par Mellars et ses collaborateurs en bordure ouest du palier sud et large d'environ $20 \mathrm{~cm}$ au niveau de la couche moustérienne, était réduite, voire inexistante, à hauteur des niveaux supérieurs B1 à B3 où, inévitablement, les deux limites (celle de Bailleau et celle de Delporte) ont dû à peu près coïncider. II est possible que Delporte, à un certain moment de ses travaux, ait perçu la limite méridionale de son palier sud comme une interface verticale entre le déblai Bailleau et les dépôts en place préservés au-delà et que les photos aient été prises pour en témoigner. Mais, si cela est le cas, les photographies montrent que le profil recoupe encore des dépôts perturbés, au moins dans leur partie supérieure.

Ces observations sont en contradiction avec la mention de Delporte de cinq niveaux rougeâtres riches en matériel et interprétés comme des niveaux d'occupation " nettement distincts par leur coloration" (Delporte et al. 1999 : 18). Mellars et alii (2007 : 3661) pensent que " cette séquence stratigraphique claire et tranchée est totalement contradictoire avec toute hypothèse de déblais du XIX ${ }^{e}$ siècle " ${ }^{7}$. Ils se trompent. Ceux qui ont été confrontés au problème d'avoir à distinguer des déblais de dépôts in situ, savent que les sédiments remaniés se caractérisent, bien souvent, par une succession de lentilles aux caractères chromatiques contrastés (cf. Texier et al. 2004).

\section{3 - Bailleau contra Delporte et Mellars et alii}

On notera tout d'abord que l'appréciation faite par Mellars et alii de la zone concernée par les fouilles du $\mathrm{XIX}^{\mathrm{e}}$, est en contradiction avec le témoignage de Delporte et l'on constate qu'il s'agit d'une erreur manifeste lorsqu'on considère la façon dont, selon eux, auraient été menées ces fouilles anciennes : elles auraient préservé la partie centrale du palier sud pour se limiter à une tranchée étro ite creusée le long de la paroi ouest. Cette solution est non seulement improbable, mais tout simplement impossible étant donnée l'étroitesse d'une telle tranchée et les contraintes spatiales environnantes.

Connaissant le mode opératoire des fouilleurs du $\mathrm{XIX}^{\mathrm{e}}$ siècle, le fait que Bailleau ait laissé intacte une partie importante du remplissage de la grotte effondrée, laisse sceptique. Que ce témoin se place, comme cela peut être déduit des indications topographiques laissées par Delporte (cf. fig. 1-3), juste après l'entrée très étroite (largeur inférieure à un $1,5 \mathrm{~m}$ ) qui permet d'accéder au site depuis le chemin, apparaît encore plus invraisemblable. En effet, ce témoin aurait fait obstacle à une évacuation efficace des sédiments fouillés dans les parties profondes du gisement, à l'emplacement où Delporte a reconnu un remblaiement lié aux fouilles du XIX ${ }^{\ominus}$ siècle (fig. 7).

Bailleau lui-même (1869 : 14) a laissé des indications concrètes sur l'extension et la profondeur des niveaux archéologiques qu'il a fouillés : " cet espace de $6 \mathrm{~m}$ de long sur $4 \mathrm{~m}$ de large " où " plusieurs occupations successives ont dû se faire " et ce sur une épaisseur " de plus d'un mètre" (fig. 8). Ces observations peuvent être confrontées au profil longitudinal de Delporte (fig. 1a), lequel indique la présence du rocher immédiatement à l'ouest des déblais du XIX ${ }^{\circledR}$ siècle qu'il a traversés. De cette comparaison, il ressort une conclusion incontoumable : le lieu où Delporte a reconnu sa succession " intacte " de niveaux rougeâtres (fig. 9) coïncide avec le seul endroit du site où peut se placer un espace avec les dimensions données par Bailleau pour le "foyer» aux riches couches archéologiques qu'il a fouillées et dont la base se place à hauteur du contact Moustérien/Châtelperronien.

La présence de dépôts châtelperroniens in situ dans la grotte des Fées, où que ce soit dans la tranchée de 1952, est donc impossible. L'affirmation du contraire par Mellars et alii. soulève une question évidente. S'il n'a pas fouillé complètement le riche foyer de la zone d'entrée, alors qu'aurait fait Bailleau à la grotte des Fées, après ses tentatives infructueuses de retrouver des témoins d'occupations préhistoriques dans les deux autres cavités ? Quand bien même, si Bailleau n'avait pas achevé la fouille de ce secteur lorsqu'il rédige sa publication de 1869, on sait qu'il continua à travailler sur le site dans les années suivantes, jusqu'en 1872. Est-il alors raisonnable de croire que des témoins importants auraient subsisté aprés plusieurs années d'exploitation d'un aussi petit secteur?

\section{4 - Incohérences dans l'interprétation d'Henri Delporte}

Cette incohérence entre les indications topographiques de Delporte et son interprétation stratigraphique est trop importante pour que lui-même, dans une certaine mesure, ne l'ait pas perçue. Une fois reconnues et situées les limites de la fouilles Bailleau telles qu'il les a placées sur son plan de 1954, Delporte aurait dû réaliser que les dépôts rencontrés dans la partie centrale du site étaient remaniés et, en particulier, que les " horizons rougeâtres " situés entre les repères topographiques 2 et 3 du profil longitudinal (c'est-à-dire la section sud de la tranchée de 1952, cf. fig. 1 à 3) ne pouvaient pas être des couches châtelperroniennes in situ comme il l'avait d'abord pensé. Qu'il ait envisagé cette possibilité, est suggéré par le paragraphe final de son rapport de fouilles pour l'année 1954 : “ les fouilles ont porté sur une

(7) "this clear and sharply defined stratigraphic sequence is totally inconsistent with any version of the 19th-century backdirt hypothesis" 

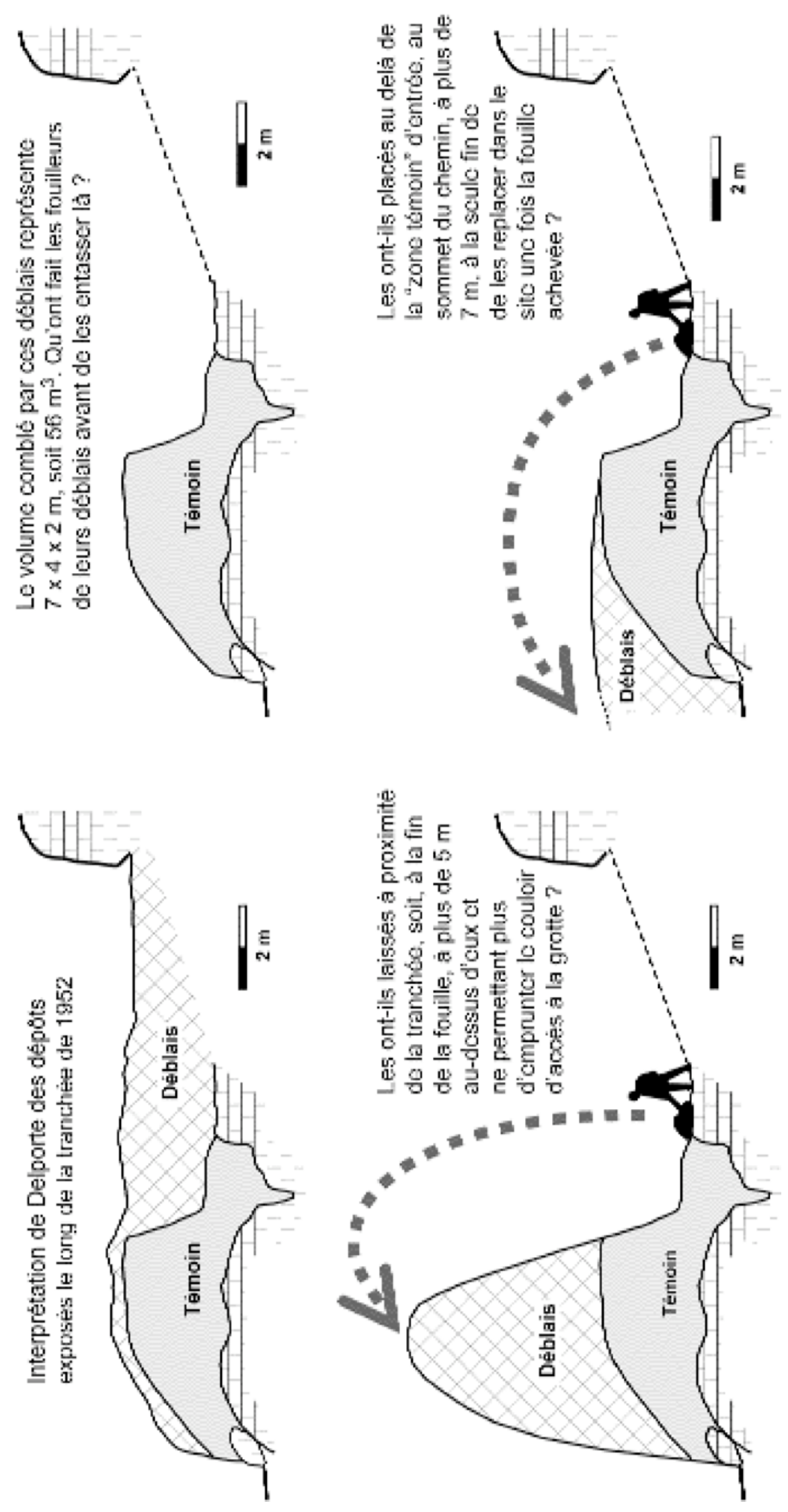

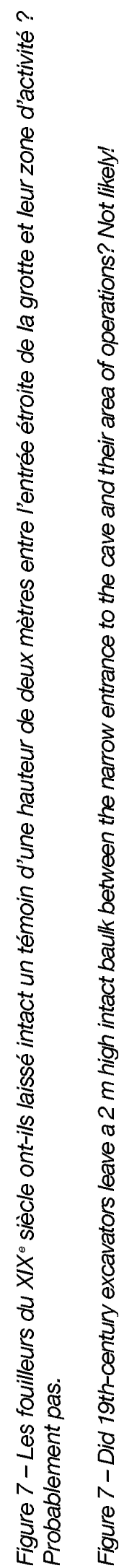


DF. ChatFa.PERBOS.

13

Les sillons traces par les dents humaines sont faciles à distingune de ecux laisgés sur les mênes ossomentx par les carnassiers. Sur la mechoire qui nous accupe, le sillon tracé par la dent commenes:

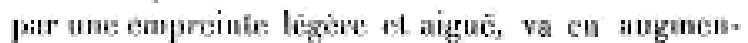
taat, pais se lermine brusquement. d'une manière large of earrie. Les supureinteg des dents de ear. nassicrs, nu contraire, n'oflren! qu'un sillon plus ou moins profond, mais tonjours aigu at moins large que dans los conpreintes dont je parle ; son t́tendue cat ausoi ordinairement plas bonsidérable.

felle mahoirt d'auroth est une pièe des plus rares, écst la seule que jasi rencontrée présentuni

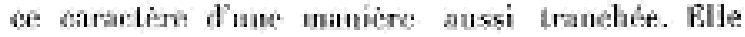
prouve non-sculement lis presenex certaine de l'homme dans ces cavernes, mais clle m'explique pourquoi, sur les eraines huenains, frouvis dins less sépultares de l'ágo des eavernes. les dents incisives sont toujours plus usées que les autres. On dit pour expliquer estle particulurité que éçwit parce que efs penplades midehaient sur lears thenis ineisives, jc crojs pouvoir ajouter quas c'était sussi parce qu'olles rangeaient les as aves elles.

3. Foyer (plus à l'Est). Jarrive å la partie la plas inlíressinte das nun Iravail, quoique je n'ai fnit que ylance en cet cnuroit, dans les fouilles que M. Poirrier $\checkmark$ avait fait exérater andírieuremonf; c'est. nevore lit que j'ii recuilli les objefs les plus nornbreux et les plus précieux.

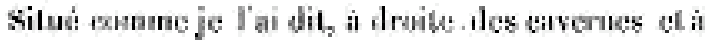
un niveau supéricur, ect emplacenent, entourć de rothers, stajt parfuitement ehoisi, en ce qu'il ctait
14

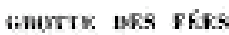

proligüdes venis du nord par le cotcuu qui s'élève serriêtre lui ; domiuant les grolles, il était admirable-

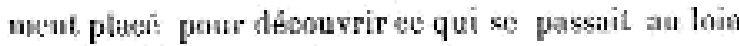
dans la vallée et devait. strvir de poste avancé pour veiller sur l'ennemi.

Pendaut longtempis l'homme y a อ Eabli son kejen

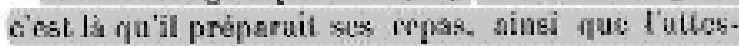

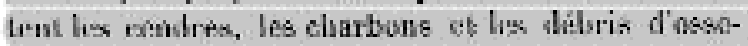
ments rongcis, qu'sin y trouve aceuinules sur unt: épaisear de plus d'un miekr. Plusieurs oecupations suecessiven ont ilu set faire dans cet cepace de six wetres de longr sur quatie de large; on reacontre en effel, a sliffirentes profondeure, en fovillani. fo sol, des plaques de sehiste juxlipusises equi ont servi à l'edification des foyers. C'est on remunt ecs eendres all cos débris que j’ai recueilli, outre les instrumeuts the silex qui les avaient tul'x, tes vestigers de fenus les. animas $x$ dont l'homme a fait sa nourriture pendan! vetic époyue si longane et si reculée.

Jy ai rencontré toute la faune Lrouvée dins las cavernes, cutre autres plusieurs defensós de manmouth, dont lane asec2 bicu conservéc avait plus de deux metres de long, sur 0,30 s:entimètres de dinmelre is sa hase, brisce di se recine, je n'en ai pts

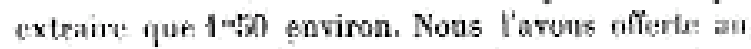
ınuséc imperial de Suint-Germain-en-Laye. C'est aussi parmi ces déloris que j'ini resureilli des quantitis cousidérables de silex, trilats, nuclẹi et instrumeuts enirers, ainsi que plusieurs objets on as on en ivoire travaille. Ces restes, d'uncindustrie primitive, prourent qu'une fois le repias achevé, l'homme demenrait encore press du feu qu'il avait ullumé of passint

Figure 8 - Reproduction du texte de Bailleau (1869 : 13-14) faisant mention du travail réalisé à la grotte effondrée.

Figure 8- Fac-simile reproduction of Bailleau's (1869: 13-14) account of the excavation work that he carried out at the Grotte Effondrée. 


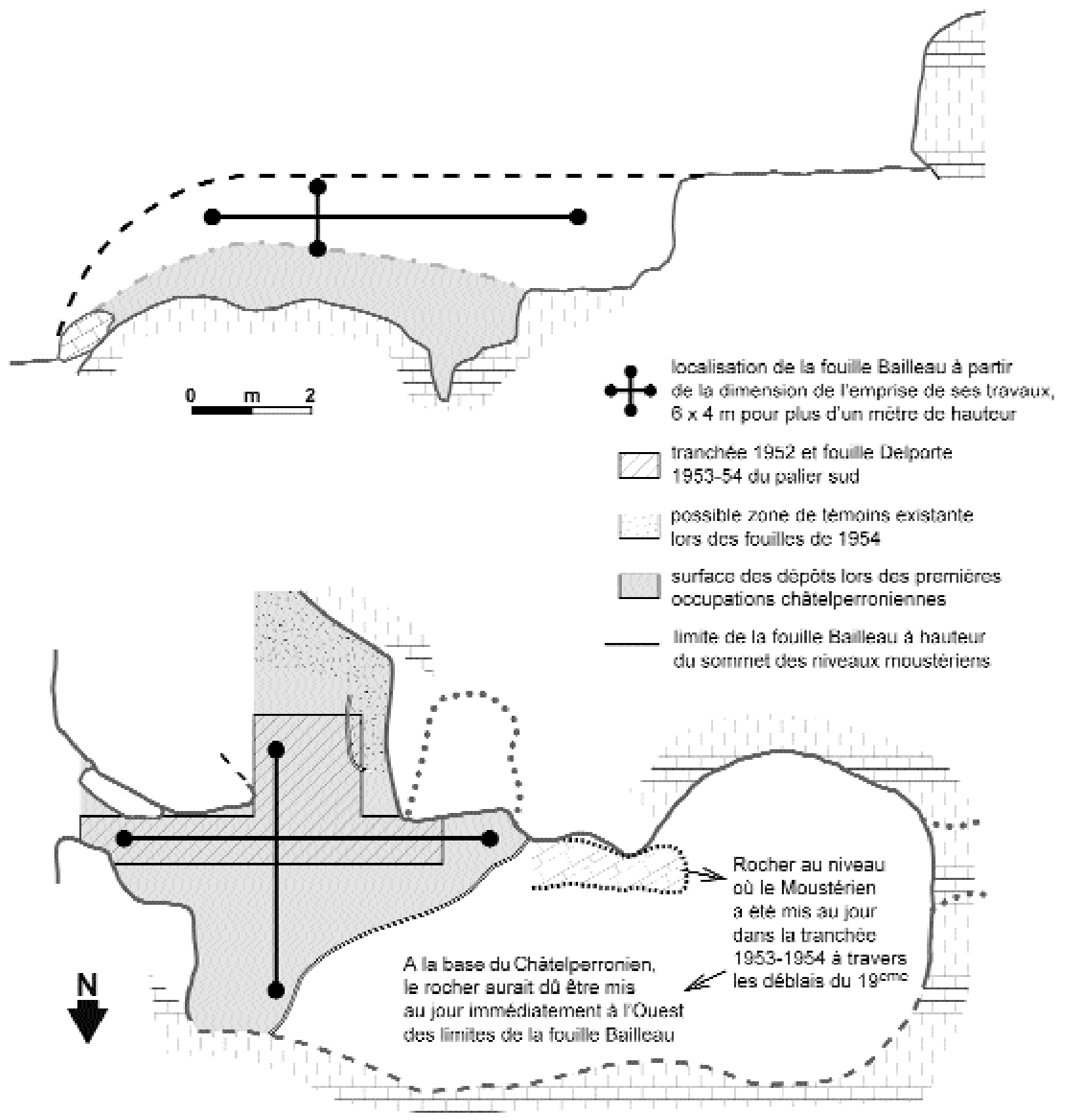

Figure 9 - Report de l'emprise de la fouille Bailleau en croisant les indications portées dans la publication de ce dernier (Bailleau 1869) avec les indications du plan de Delporte. Cette figure montre que la tranchée Delporte de 1952 et les fouilles 1953-1954 du palier sud ne peuvent avoir que traversé les déblais du XIX siècle. Dans la partie centrale de la grotte, des témoins du remplissage ne peuvent avoir été préservés qu'entre le profil ouest du palier sud et la paroi de la cavité. Le trait discontinu représente la surface hypothétique du sol à l'époque de Bailleau, sur la base des indications de Delporte (cf. fig. 1).

Figure 9 -Extention of Bailleau's excavations based on Bailleau's data (Bailleau, 1869) and Delporte's map. Obviously, Delporte's 1952 trench and 1953-1954 palier sud excavations could only have been made into 19th-century backfill. In this central part of the Grotte Effondrée, intact Châtelperronian remnants only could have existed between the western profile and the cave wall. Using Delporte's (cf. Fig. 1) as an approximation, the dashed line represents the hypothetical ground surface at the time of Bailleau. 


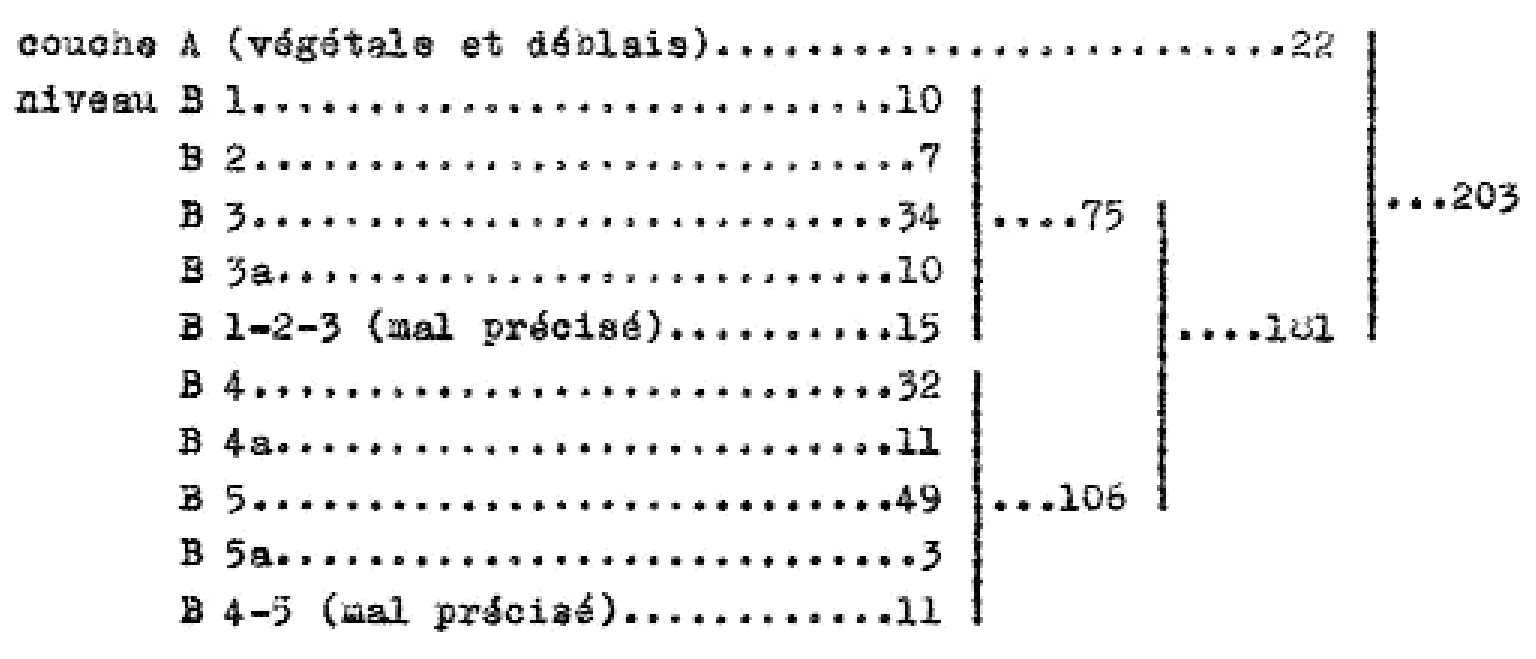

Figure 10 - Décompte d'Henri Delporte (n.d. : 28) des pièces retouchées par unités stratigraphiques. Le terme " mal précisé " traduit une situation stratigraphique, non pas une catégorie typologique. Vu le contexte, la catégorie "B1-2-3" ne peut correspondre qu'à la catégorie "B1-3" observée sur objets marqués et dans des étiquettes de sacs à os portant aussi mention de l'année 1962.

Figure 10 - Delporte's (n.d.: 28) table of retouched tool-types per stratigraphic unit in the Châtelperronian levels of his excavations at the Grotte des Fées. The "poorly defined" [mal précisé] qualification refers to stratigraphic provenience, not tool-typology. The "B1-2-3" unit, , must correspond to the "B1-3" unit present in find labels with a 1962 excavation date.

station, sinon vidée, tout au moins ne contenant plus que des lambeaux de couches vierges de caractère périphérique, par conséquent infiniment moins riches que la zone centrale qui contenait les importants foyers étudiés par Bailleau vers 1870 " (Delporte $1955: 84$ ).

Cela semble avoir échappé à l'attention de Mellars et alii. II est pourtant manifeste qu'il y a contradiction entre l'affirmation de Delporte et le profil longitudinal produit dans le même rapport où apparaissent, dans la zone centrale du site, d'importants témoins de couches réputées non perturbées (cf. fig. 1). Que Delporte en ait été conscient, en dépit de l'apparente constance de son interprétation stratigraphique, peut expliquer l'absence d'une publication de détails et les réserves exprimées, en a parte, par le fouilleur quant à l'utilisation de la grotte des Fées en tant qu'exemple fiable d'interstratification Châtelperronien-Aurignacien (F. Bon, communication personnelle à J.-G. Bordes).

\section{4 - DATES RADIOCARBONE}

Mellars et ses collaborateurs considèrent que la cohérence des datations ${ }^{14} \mathrm{C}$ est leur meilleur argument :
"La cohérence manifeste (...) des huit dates obtenues pour les niveaux châtelperroniens les plus hauts (B1 à B3) est particulièrement significative. Mise à part leur cohérence propre, pourquoi aucun échantillon n'a donné de date dans l'intenalle 39 - 40 ka BP, ce qui aurait inévitablement dû se produire si ces échantillons avaient été recueillis dans des déblais provenant de la fouille des niveaux châtelperroniens in situ sous-jacents B4 et B5 ? "8 (p. 3659-3660).

\section{1 - Provenance des échantillons}

Mellars et alii (p. 3659) considèrent que les huit échantillons en question proviennent de l'ensemble des couches B1 à B3. Toutefois, leur étiquetage exact est : " B1-3" (ce qui est correctement indiqué dans la première publication de Gravina et al. 2005, tabl. 1). Cette mention, tout comme celle de B4-5 pour les niveaux inférieurs, désigne des pièces dont la provenance stratigraphique est imprécise (fig. 10), et non pas une association de pièces provenant de divers niveaux initialement individualisés par H. Delporte. De plus, lorsqu'une indication d'année est donnée sur les pièces ainsi marquées, il s'agit de l'année 1962, et cela est également le cas pour les étiquettes en papier contenues dans les sacs de pièces osseuses. Ces deux types d'indication de provenance ne

(8) "The striking consistency (...) of the eight dates secured for the upper Chatelperronian levels (B1-B3) is particularly significant. Apart from their internal consistency, why is it that not one of these samples produced a date in the range of 39,00040,000 BP, which one would inevitably expect if these samples derived from the excavation backdirt of the underlying, in situ Chatelperronian levels in layers B4 and B5?" 

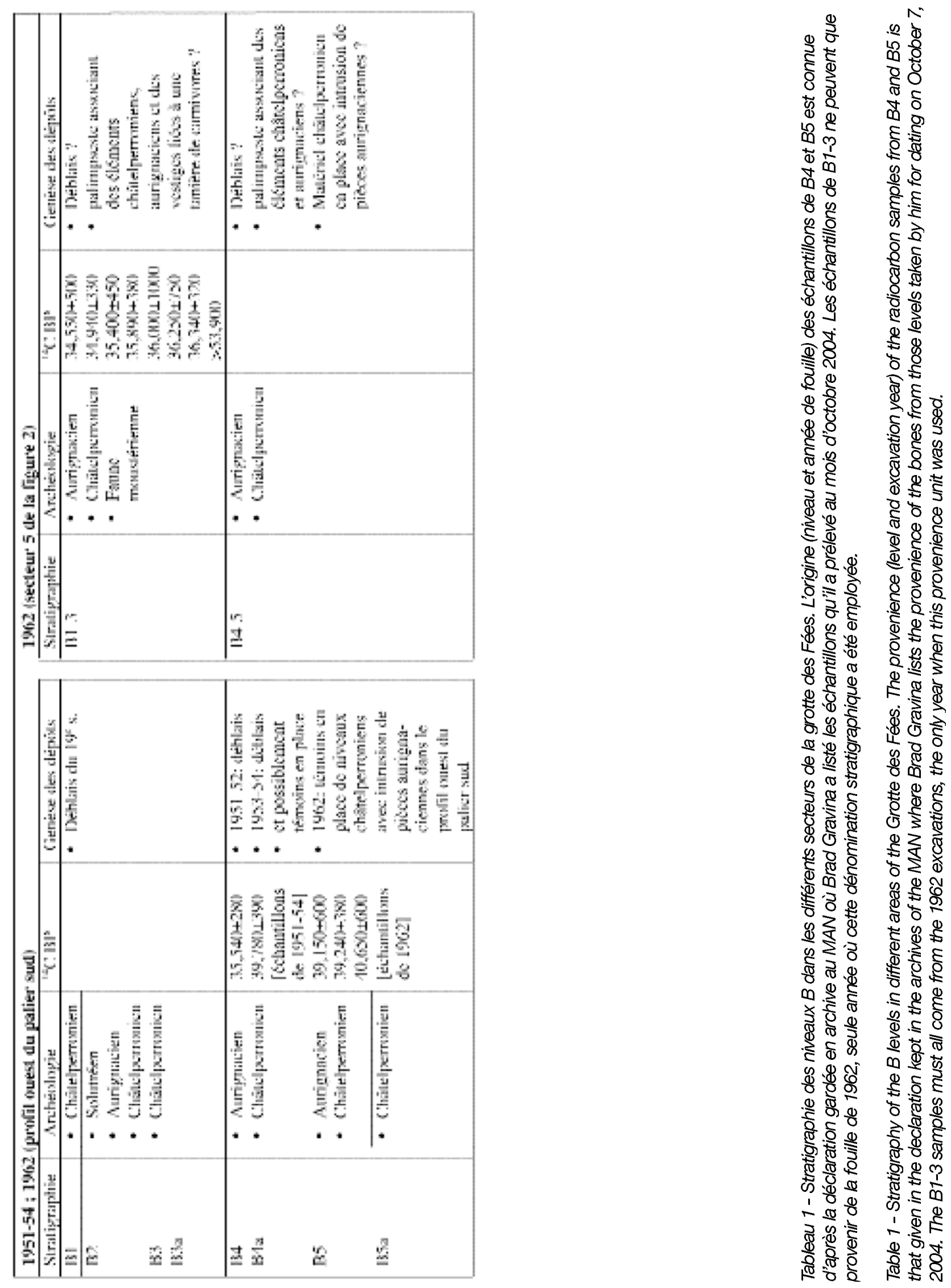


\begin{tabular}{|l|c|c|}
\hline & B1-3 & B4-5 \\
\hline Pièces chătelperroniennes & & \\
Lamos bipolairos brutos & 3 & 1 \\
Pièces à dos & 8 & 5 \\
Troncatures & 1 & 1 \\
Pièces aurignaciennes & & \\
$\quad$ Grattoir aurignacien & 1 & - \\
Lamello Dufour & - & 1 \\
\hline Autres & 39 & 17 \\
\hline Total des elements lithiques & 52 & 25 \\
os & 162 & - \\
\hline
\end{tabular}

Tableau 2 - Décompte des pièces recueillies dans les niveaux B1-3 et B4-5 de la grotte des Fées par Delporte en 1962 (selon Zilhão et al. 2006, et données inédites ; les huit échantillons datés de B1-3 ont été ajoutés au décompte des ossements).

Table 2 - Finds from the B1-3 and B4-5 levels of the Grotte des Fées (Delporte's 1962 excavations). After Zilhão et al. (2006) and unpublished data. The eight radiocarbon dated samples from B1-3 were added to the bone count.

s'appliquent ainsi qu'à des vestiges recueillis lors de l'extension de 1962 du palier sud (fig. 2-3), ce que confirme par ailleurs la mention de ces types de marquage dans le seul rapport de 1964. Cette déduction est finalement validée par un commentaire dans le manuscrit inédit de Delporte (n. d. : 56), précisant la provenance d'un grattoir aurignacien, marqué à l'encre " Ch 62 " et au crayon " B1/3" : "recueilli dans une zone où les niveaux se différenciaient mal".

Sans être ici catégorique, une lecture soigneuse des faits permet les déductions suivantes :

1- le secteur où la résolution stratigraphique était la plus mauvaise est celui qui se trouve dans le prolongement du palier sud de 1954 ;

2- le système de dénomination stratigraphique des fouilles antérieures a été utilisé en 1962 pour l'enregistrement de pièces provenant du témoin in situ préservé entre la paroi et la limite ouest de la fouille de 1954. II est, au moins, clair que la cohérence stratigraphique des trois datations de pièces issues du niveau B5, toutes recueillies en 1962 (tabl. 1), s'accorde avec l'hypothèse selon laquelle des dépôts châtelperroniens intactes (bien que contenant quelques pièces aurignaciennes) aient pu exister dans ce secteur du palier sud.

Dans tous les cas, une première conclusion des plus claires se dégage quant à la provenance des échantillons datés : la question posée précédemment par Mellars et alii est un sophisme. Si aucune date comparable à celle de la série B4-5 n'a été obtenue pour les niveaux supérieurs B1, B2 et B3, c'est simplement parce qu'aucun échantillon comespondant n'a été daté.

\begin{tabular}{|c|c|c|c|c|}
\hline & \multicolumn{2}{|l|}{$\overline{O s}$} & \multicolumn{2}{|c|}{ Elénuents lithiques } \\
\hline Niveuu & $\mathrm{N}$ & ${ }_{0}$ & $\mathrm{~N}$ & $\mathscr{w}$ \\
\hline $\mathrm{B} 1$ & 2 & 1 & 61 & 8 \\
\hline B) & 2. & 1 & 68 & 9 \\
\hline B3 & - & - & 76 & 10 \\
\hline B.3а & 3 & 1 & 6.3 & 8 \\
\hline $\mathrm{R} 4$ & 4 & 1 & 164 & 27. \\
\hline B4a & 7 & 2 & 73 & 10 \\
\hline LS5 & 5 & 2 & 151 & 20 \\
\hline B.5a & 127 (a) & 11 & 16 & 2 \\
\hline $\mathrm{B} \perp 3$ & $162(\mathrm{~b})$ & 52 & 52 & 7 \\
\hline L4-5 & - & - & 25 & 3 \\
\hline 'Total & 312 & 1001 & 749 & $100)$ \\
\hline
\end{tabular}

(a) quatre piéces hrûlés, un os ronge par des carnivores et quatre-vingt-quatre os préwentant des traces de régurgitulion (b) guatre pièces brûlées, deux os rongés par des carnivores et vingt deux os présentant des traces de régurgitation. (a) 4 burnt, 1 carnivors-gnawed, 84 respurgitaled pieces.

(b) 2 burnl, 2 carnivore-gnawed. 22 resgurgitaled pieces.

Tableau 3 - Ossements (dents exclues) et éléments lithiques recueillis dans les différents niveaux $B$ de la grotte des Fées, fouilles H. Delporte (selon Zilhão et al. 2006, tableau 2 ; les treize échantillons datés ont été ajoutés au décompte des ossements).

Table 3 - Bone (teeth excluded) and lithic artifact finds in the different $B$ levels of Delporte's excavations at the Grotte des Fées. After Zilhão et al. (2006: Table 2). This inventory relates to the collections of the MAN. The 13 radiocarbon dated samples were added to the bone counts

\section{2 - Signification des dates obtenues pour B1-3}

Pour discuter la signification véritable des dates obtenues pour le niveau $B 1-3$, il est nécessaire de considérer le contexte archéologique immédiat et de garder à l'esprit la nature des échantillons. La composition des séries lithiques recueillies en B1-3 et B4-5, plutôt pauvres (tabl. 2), autorise trois interprétations (tabl. 1) :

1- les deux niveaux sont in situ et correspondent à un palimpseste où se mêlent des vestiges aurignaciens et châtelpenoniens, cette association pouvant être d'origine syn-sédimentaire ;

2- le niveau B4-5 est un dépôt châtelperronien intact, la lamelle Dufour recueillie dans cet horizon étant intrusive, tandis que le niveau B1-3 est un palimpseste associant vestiges aurignaciens et châtelperroniens ; 


\begin{tabular}{|c|c|c|c|c|c|}
\hline Site & Niveau & Culture/Fossile & $\begin{array}{l}\text { Référence de } \\
\text { laboratoire }\end{array}$ & Résultat BP & calBP (2s) \\
\hline Kloine Foldhofor & & & & & $42290-$ \\
\hline Girolte & Déblais & Néandertalien & FTH 19660 & 392401670 & 44650 \\
\hline & Mandibule & & & $34950 /+990 /-$ & $38410-$ \\
\hline Oase & & Homme modeme & OxA-11711/GrA-6165 & 890 & 42490 \\
\hline Grotte des Fées & B5 & Chátelperronien & OxA-13621 & $40650 \pm 600$ & $\begin{array}{l}43040- \\
45560 \\
42770-\end{array}$ \\
\hline Grotte dos Fócs & B4 & Chätolperronicn & OxA-14319 & $39780 \pm 390$ & $\begin{array}{r}44730 \\
47450\end{array}$ \\
\hline Grotte des Fées & B5 & Chatelperronien & $0 \times A-14320$ & $39240 \pm 380$ & $\begin{array}{l}44490 \\
42270-\end{array}$ \\
\hline Grotte dos Fóos & B5 & Chätc|perronicn & OxA-13622 & $39150 \pm 600$ & $\begin{array}{l}44590 \\
41040\end{array}$ \\
\hline Grotte des Fees & $\mathrm{B} 4$ & Chatelperronien & OxA-14318 & $36540 \pm 280$ & $\begin{array}{l}42040 \\
43910-\end{array}$ \\
\hline Roc-de-Combe & 8 (square K9) & Châtelperronien & Gif-101265 & $45100 \pm 2100$ & $\begin{array}{l}53270 \\
42110\end{array}$ \\
\hline Roc de Combe & 8 (square K9) & Chãtelperronien & Gill 101266 & $40000 \cdot 1300$ & $\begin{array}{l}45830 \\
42230-\end{array}$ \\
\hline Roc-de-Combe & 8 (square K9) & Châtelperronien & Gif-101264 & $39540 \pm 970$ & 45030 \\
\hline Grotte XVI & B & Chätelporronion & AA-2674 & $>39800$ & $\frac{-}{40760}$ \\
\hline Grotte XVI & $B$ & Chatelperronien & AA- 2997 & $38100 \pm 1670$ & $\begin{array}{l}45040 \\
37890-\end{array}$ \\
\hline Grotte XVI & B & Châtelperronien & GifA-95581 & $35000 \pm 1200$ & $\begin{array}{l}42730 \\
41600-\end{array}$ \\
\hline Combe Sauniêre & $x$ & Chătelperronien & OxA-650.3 (tripeptide) & $38100+1000$ & $\begin{array}{l}44320 \\
39800-\end{array}$ \\
\hline Abri Dubalen & $\mathrm{EBC} 2$ & Chátelperronien & GifA-101045 & $36130 \pm 690$ & $\begin{array}{r}42920 \\
40280-\end{array}$ \\
\hline La Quina, aval & 4 & Chătelperronien & OxA-10261/.y-1357 & $35950+450$ & $\begin{array}{l}42600 \\
38380-\end{array}$ \\
\hline Caune de Belvis & 7 & Chatelperronien & AA-7390 & $35425 \pm 1140$ & 42900 \\
\hline Grotte des Fées & $\mathrm{B} 1-3$ & $\begin{array}{l}\text { Taniefe de } \\
\text { Carnivores } \\
\text { Tanière de }\end{array}$ & OxA-13620 & $=53900$ & $4 \overline{470}$ \\
\hline Grotte dos Fices & B1-3 & $\begin{array}{l}\text { Camivorcs } \\
\text { Taniere de }\end{array}$ & OxA-14165 & $36340 \pm 320$ & $\begin{array}{l}42390 \\
39900-\end{array}$ \\
\hline Grotte des Fées & $\mathrm{B} 1-3$ & $\begin{array}{l}\text { Camivores } \\
\text { Taniere de }\end{array}$ & OxA-13724 & $36250 \pm 750$ & $\begin{array}{l}42980 \\
38840-\end{array}$ \\
\hline Grotte des Fées & $\mathrm{B} 1-3$ & $\begin{array}{l}\text { Carnivores } \\
\text { Tanicie de }\end{array}$ & OxA-13723 & $36000 \pm 1000$ & $\begin{array}{l}43200 \\
40390-\end{array}$ \\
\hline Grotte des Fêes & $\mathrm{B} 1-3$ & $\begin{array}{l}\text { Camivares } \\
\text { Taniere de }\end{array}$ & OXA-13618 & $35890+380$ & $\begin{array}{l}42510 \\
39190-\end{array}$ \\
\hline Grotte des Fées & $\mathrm{B} 1-3$ & $\begin{array}{l}\text { Carnivores } \\
\text { Tanicice de }\end{array}$ & OxA-13619 & $35400 \pm 450$ & $\begin{array}{l}42550 \\
39020\end{array}$ \\
\hline Girctte des Fées & B1-3 & Camivores & CxA-14166 & $34940+330$ & 42220 \\
\hline Grotte des Fees & $\mathrm{B} 1-3$ & $\begin{array}{l}\text { Taniere de } \\
\text { Comivores }\end{array}$ & OxA-13617 & $34550 \pm 500$ & $\begin{array}{l}38790- \\
41990 \\
\end{array}$ \\
\hline Caminade & $\mathrm{F}$ & Aurignacien I & GifA- 97186 & $35400 \pm 1100$ & $\begin{array}{l}38450- \\
42850 \\
37490\end{array}$ \\
\hline Roc-de-Cornbe & $7 \mathrm{c}$ & Aurignacicn I & OxA-1263 & $34800 \pm 1200$ & $\begin{array}{l}42730 \\
35830-\end{array}$ \\
\hline Roc-de-Combe & $7 b$ & Aurignacien I & OxA-1262 & $33400 \pm 1100$ & $\begin{array}{l}42230 \\
36710-\end{array}$ \\
\hline Le Flageolet I & $\mathrm{XI}$ & Aurignacien I & GifA-95559 & $34300 \pm 1100$ & $\begin{array}{l}42030 \\
35300-\end{array}$ \\
\hline Le Flageolet I & $\mathrm{XI}$ & Aurignacien I & OXA-598 & $33800+1800$ & $\begin{array}{l}42940 \\
35270-\end{array}$ \\
\hline Le Flageolet I & $\mathrm{XI}$ & Aurignacien I & GifA-95538 & $32040 \pm 850$ & $\begin{array}{r}39750 \\
38820-\end{array}$ \\
\hline Tuto de Camalhot & $70-807$ & Aurignacien I & GitA 99093 & $34750 \pm 570$ & $\begin{array}{l}42140 \\
35740\end{array}$ \\
\hline Tuto de Camalhot & $70-80 ?$ & Aurignacien I & GifA-99674 & $32180 \pm 570$ & $\begin{array}{l}39540 \\
36670-\end{array}$ \\
\hline Combe Saunićrc & VIII & Aurianacicn I & OxA-6507 & $34000 \pm 850$ & $\begin{array}{l}42430 \\
36410\end{array}$ \\
\hline Grolle des Hyenes & $2 \mathrm{DF}$ & Aurignacien I & GіГЛ SM 11034 & 336001240 & $\begin{array}{l}41570 \\
36210\end{array}$ \\
\hline Grotte des Hyónes & $2 \mathrm{DE}$ & Aurignacicn I & GifA-98105 & $32410 \pm 370$ & $\begin{array}{l}39450 \\
36400-\end{array}$ \\
\hline Grolte des Hyenes & $2 \mathrm{~F}$ & Aurignatcien I & GІГЛ SM 11035 & $31950 \cdot 160$ & $\begin{array}{l}37400 \\
35930-\end{array}$ \\
\hline La Quina, aval & 3 & Aurignacien I & OxA-6147/Ly-256 & $32650 \pm 850$ & 40130 \\
\hline
\end{tabular}

Tableau 4 - Dates ${ }^{14} \mathrm{C}$ AMS (toutes sur ossements) de la grotte des Fées, du Châtelperronien, des sites français de l'Aurignacien 1 et des ossements humains datés et associés de façon fiable à ces deux technocomplexes. Le logiciel de calibration utilisé est CalPal Weninger et Jöris 2007).

Table 4 - AMS ${ }^{14} \mathrm{C}$ radiocarbon results (all on bone) for the Grotte des Fées, the Châtelperronian, the French Aurignacian I, and directly dated human fossils conceivably associated with these technocomplexes.

Sources: Bon 2002; Bordes 2002; Dujardin 2001; Henry-Gambier et al. 2004; Gravina et al. 2005; Mellars 1999, Rigaud 2001; Schmitz et al. 2002; Trinkaus et al. 2003. Calibration uses the CalPal software (Weninger and Jöris 2007). 
3- le secteur à pauvre résolution stratigraphique correspond, de la base au sommet, à une zone de dépôts perturbés (e.g. déblais).

Quelle que soit l'hypothèse retenue, cette séquence ne saurait être interprétée comme une interstratification car, par définition, et quand bien même les niveaux B1-3 et B4-5 auraient été en place, une interstratification implique trois niveaux distincts alors qu'ici il n'y en a que deux.

La grotte des Fées illustre bien une pratique douteuse dans l'attribution chronologique des couches archéologiques. Lorsque que l'on date des dépôts intacts, et à moins d'indications claires d'une sédimentation très lente où d'un palimpseste associant des événements distincts séparés par des hiatus importants, la faune doit être grossièrement contemporaine des vestiges lithiques associés et ce, quel que soit l'agent d'accumulation considéré. Mais, s'il est possible que les sédiments aient été perturbés, ce postulat ne peut encore être admis qu'à la condition de ne retenir que les os présentant des traces indubitables d'une intervention anthropique (par exemple des stries de découpe). Gravina et al. (2005) ne font aucune mention de telles modifications anthropiques sur les ossements datés de la grotte des Fées. Lorsqu'on considère les os issus du niveau B1-3, $16 \%$ des cent cinquante quatre pièces analysables portent des traces de régurgitation ou de rongement par des carnassiers (tabl. 3). Cette proportion suggère fortement que l'ensemble de la série, dont les os datés, est lié à une activité de camivores plutôt qu'à une activité humaine, une conclusion que Mellars et al. ne contestent pas. Mais dans ce cas, la contemporanéité des vestiges lithiques et osseux du niveau B1-3 n'est qu'une hypothèse à démontrer, puisque les ossements ont pu se retrouver associés aux vestiges châtelpemoniens contenus dans des dépôts préexistants, à la suite d'une occupation ultérieure par des camivores.

L'emprise des fouilles Bailleau indiquée par Delporte dans son plan de 1954 n'exclut pas que les dépôts rencontrés en 1962 dans le prolongement du palier sud, ont été, au moins en partie, en place. Ironie du sort, la médiocre résolution stratigraphique notée par Delporte (probablement parce qu'il n'a pas retrouvé là la succession de couches aisément distinguables par leur contraste de couleur) peut bien être un argument en faveur de cette hypothèse. Et même si cela est le cas, l'association d'os accumulés par des camivores avec des vestiges châtelperroniens peut encore refléter une apparence de contemporanéité qui reste néanmoins à établir. Parmi les possibles scénarios de formation du site portés dans le tableau 1, deux d'entre eux illustrent cette proposition : celui où le niveau B1-3 et celui où les deux niveaux B1-3 et B4-5, représentent des palimpsestes associant accidentellement des os et des silex, accumulés par des agents distincts et par conséquent, les ossements recueillis ne sauraient fournir un matériau convenable pour dater les occupations préhistoriques dont témoignent les vestiges lithiques.

Ces considérations ont trois implications sur l'interprétation des datations de la grotte des Fées (tabl. 4) :
1- pour le niveau B4, l'incertitude dans la nature et dans l'origine des échantillons, ainsi que le caractère disparate des résultats obtenus, rend impossible toute discussion fructueuse sur leur signification ;

2- pour le niveau B5, on peut admettre que les dates obtenues reflètent des moments d'occupation du site par les Châtelperroniens parce que, quel que soit l'agent d'accumulation considéré, elles sont apparemment issues d'échantillons prélevés dans un témoin grossièrement intact ;

3- pour le niveau B1-3 qui, au mieux, représente des dépôts anciens mais perturbés, les datations montrent un épisode majeur de l'occupation du site par des camivores, entre $36,340 \pm 320{ }^{14} \mathrm{C}$ BP $(\mathrm{OxA}-14165)$ et $34,550 \pm 500{ }^{14}$ C BP (OxA-13617).

$\mathrm{Si}$, au moment de l'occupation de la cavité par les camivores, la grotte n'était pas fréquentée par les hommes, les datations obtenues pour le niveau B1-3 ne font que fixer respectivement les terminus ante quem et post quem des occupations châtelperroniennes et aurignaciennes. Cette proposition est, par ailleurs, tout à fait cohérente avec le schéma général de la chronostratigraphie de la transition Paléolithique moyen / supérieur en Europe (p. ex. Zilhão 2007 : fig. 4). Le fait qu'aucun indice d'industrie proto-aurignacienne n'ait été identifié dans la collection (le matériel aurignacien reconnu n'est attribuable qu'à un Aurignacien I ou II, cf. infra) suggère également une interruption dans l'occupation humaine du secteur, en accord avec l'hypothèse selon laquelle la série de dates en B1-3 représente une période d'occupation du site par les camivores. Puisqu'il existe un recouvrement, d'une part, entre les plus anciennes dates du niveau B1-3 et le Châtelperronien et, d'autre part, entre les dates les plus récentes de ce même niveau et l'Aurignacien I, (fig. 12), on ne peut exclure que ce recouvrement soit lié à des occupations humaines. Mais, compte tenu du contexte régional, il n'y a aucune raison de penser que les dates du niveau B1-3 indiquent une occupation châtelperronienne continue tout au long de cette période. Même si les hommes ont fréquenté le site lorsqu'il servait de refuge aux carnivores, il est bien plus probable que cette présence, châtelperronienne au début et aurignacienne à la fin, a été courte et intermittente.

En résumé, les dates obtenues pour le niveau B1-3 ne soutiennent aucunement l'hypothèse d'une interstratification à l'échelle du site, pas plus qu'à l'échelle de la région.

\section{3 - Un heureux hasard}

Compte tenu de l'indigence des séries lithiques, de l'histoire taphonomique des assemblages osseux et du fait que l'indication B1-3 ne se rapporte qu'à la poursuite de la fouille du palier sud en 1962, les datations suggèrent, qu'après 36500 ans BP, le renfoncement dans la paroi sud de la salle effondrée a fonctionné comme un refuge de carnivores. Cette déduction est corroborée par la description faite par Delporte (1955 : 81, 1957 ; 457) du niveau 5a, selon laquelle cette zone paraît avoir été un emplacement de premier choix pour une tanière de carnivore, non pas après mais avant l'occupation châtelperronienne. En effet, selon Delporte, le niveau B5a 


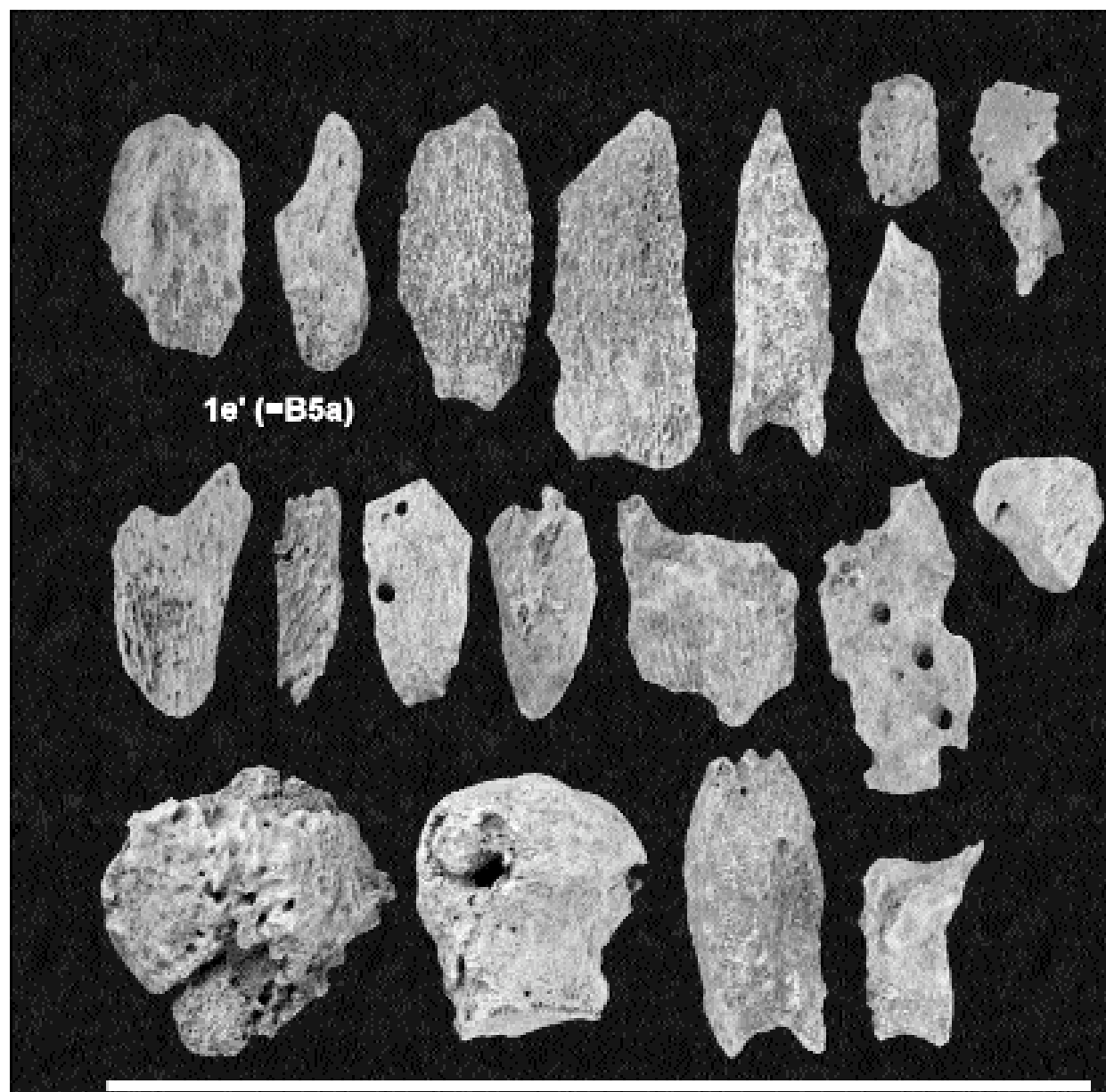

Figure 11 - Vestiges

fauniques portant des

traces de digestion par

la Hyène. Les pièces

sont issues de diffé-

rents niveaux de la

fouille Delporte à la grotte des Fées. Ces

photos montrent

l'émoussé de surface,

l'amincissement des

bords, la présence de

perforations par action d'acides gastriques qui sont typiques de ces altérations. On note la présence de pseudopoinçons ou pseudopointes identiques à ceux décrits dans des tanières pléistocènes de Hyènes ou dans les tanières actuelles en Afrique Nilla et d'Errico 2001).

Figure 11 - Faunal remains from various levels of Delporte's excavations at the

Grotte des Fées bearing features typical of hyena digestion (etched scalloped surface, thin edges, perforations produced by gastric acids), including pseudo-awls and pseudo-points such as that from $\mathrm{B5}$ a in the bottom row, similar to those reported from African and

Pleistocene hyena dens Nilla and d'Errico 2001). 


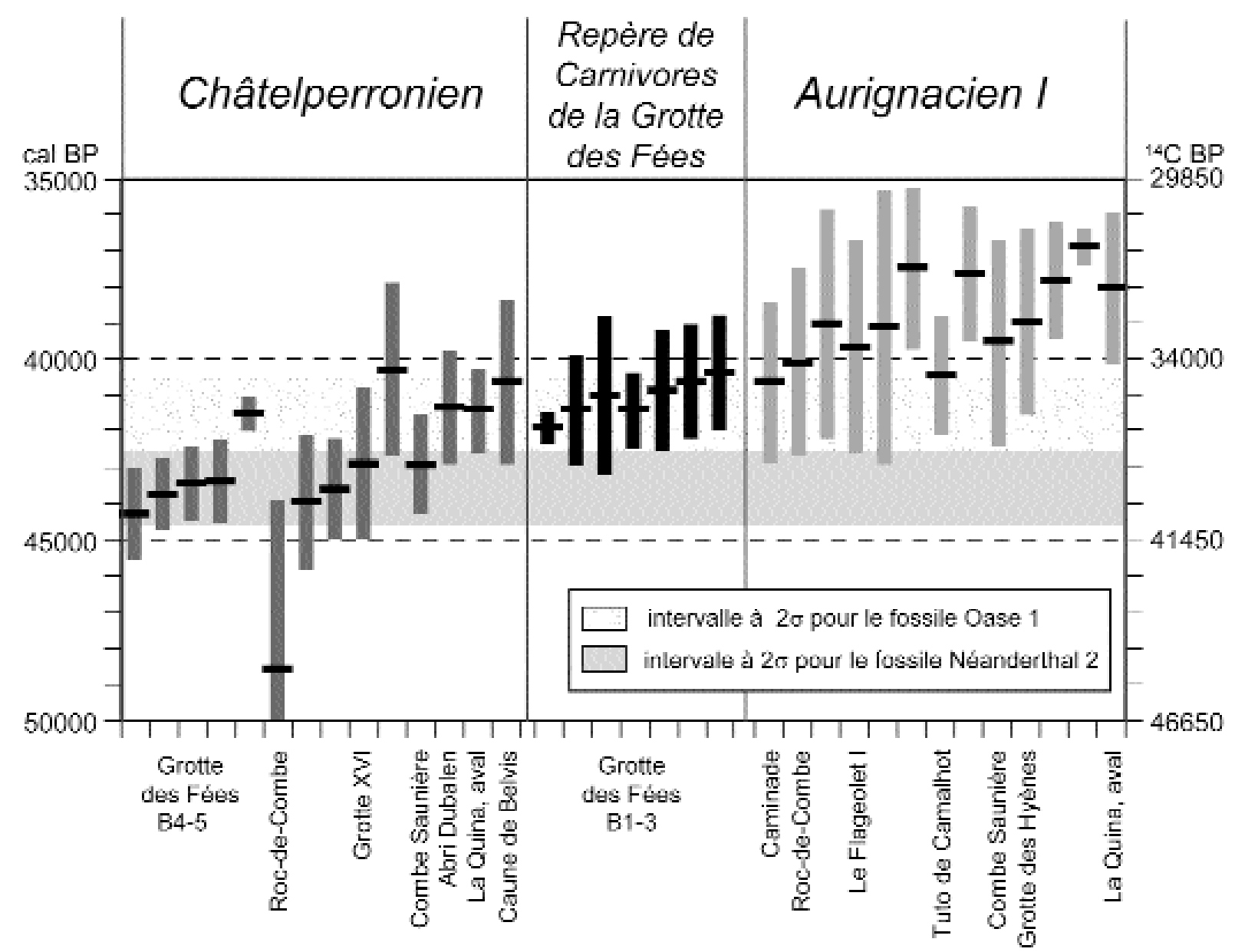

Figure 12 - Représentation, avec un intervalle de confiance de deux écarts-type, des dates portées dans le tableau 4. Les dates de l'abri Dubalen, de la Quina aval et de la Caune de Belvis n'ont pas été obtenues par ultrafiltration et peuvent, en conséquence, être sous-estimées (Higham et al. 2006). La date de la Quina aval, en particulier, est donnée pour avoir été obtenue à partir d'un os “pauvrement préservé " (Dujardin 2001). Dans le cas de la grotte XVI et de la grotte des Fées et, tenant compte des autres dates obtenues pour les même niveaux, les dates les plus récentes peuvent aussi représenter des sousestimations sans pour autant témoigner d'une longue contemporanéité avec les Aurignaciens.

Figure 12 - Two-sigma plot of the non-infinite dates in Table 4. Note that the results for Abri Dubalen, La Quina Aval and Caune de Belvis are on non-ultrafiltrated samples and, hence, likely underestimated (Higham et al. 2006); the result for La Quina Aval in particular comes from a "poorly presened" bone (Dujardin 2001). At Grotte XVI and at Grotte des Fées, bearing in mind the ensemble of results, the younger date is also an underestimation, not evidence for long-term contemporaneity with the Aurignacian.

comblait une cuvette dans le palier sud, creusée de $18 \mathrm{~cm}$ dans les niveaux moustériens sous-jacents et dans laquelle, en dépit de sa faible superficie $\left(6 \mathrm{dm}^{2}\right.$, soit un diamètre inférieur à $30 \mathrm{~cm}$ ), il trouva une concentration importante d'esquilles osseuses : 127 , soit pas moins que $41 \%$ de l'assemblage faunique de l'ensemble des niveaux B (tabl. 3).

L'utilisation de ce secteur du site essentiellement, si ce n'est exclusivement, comme une tanière de camivores pe rmet de comprendre pourquoi la perplexité de Mellars et collaborateurs vis-à-vis de notre interprétation de la stratigraphie et des dates, n'est pas justifiée (fig. 13). L'acceptation de Mellars et alii de la validité des dates du niveau B1-3 en tant que datation d'une occupation châtel- perronienne est basée sur une hypothèse implicite selon laquelle des ossements d'origine anthropique, contemporains des vestiges lithiques et représentant un " bruit de fond " châtelperronien, doivent être présents dans l'assemblage faunique de ce niveau. En conséquence, le signal chronologique correspondant aurait dû être révélé par les datations. Cependant, le secteur d'où proviennent les échantillons datés de B1-3, est situé bien au-delà de la zone où Bailleau a identifié, au centre du foyer devant la salle effondrée, les témoins d'une occupation préhistorique. II n'y a aucune raison de croire que Bailleau n'aurait pas fouillé exhaustivement ces riches niveaux. En d'autres termes, tout porte à croire que l'habitat châtelperronien ne s'étendait pas au-delà des limites de la fouille Bailleau. 

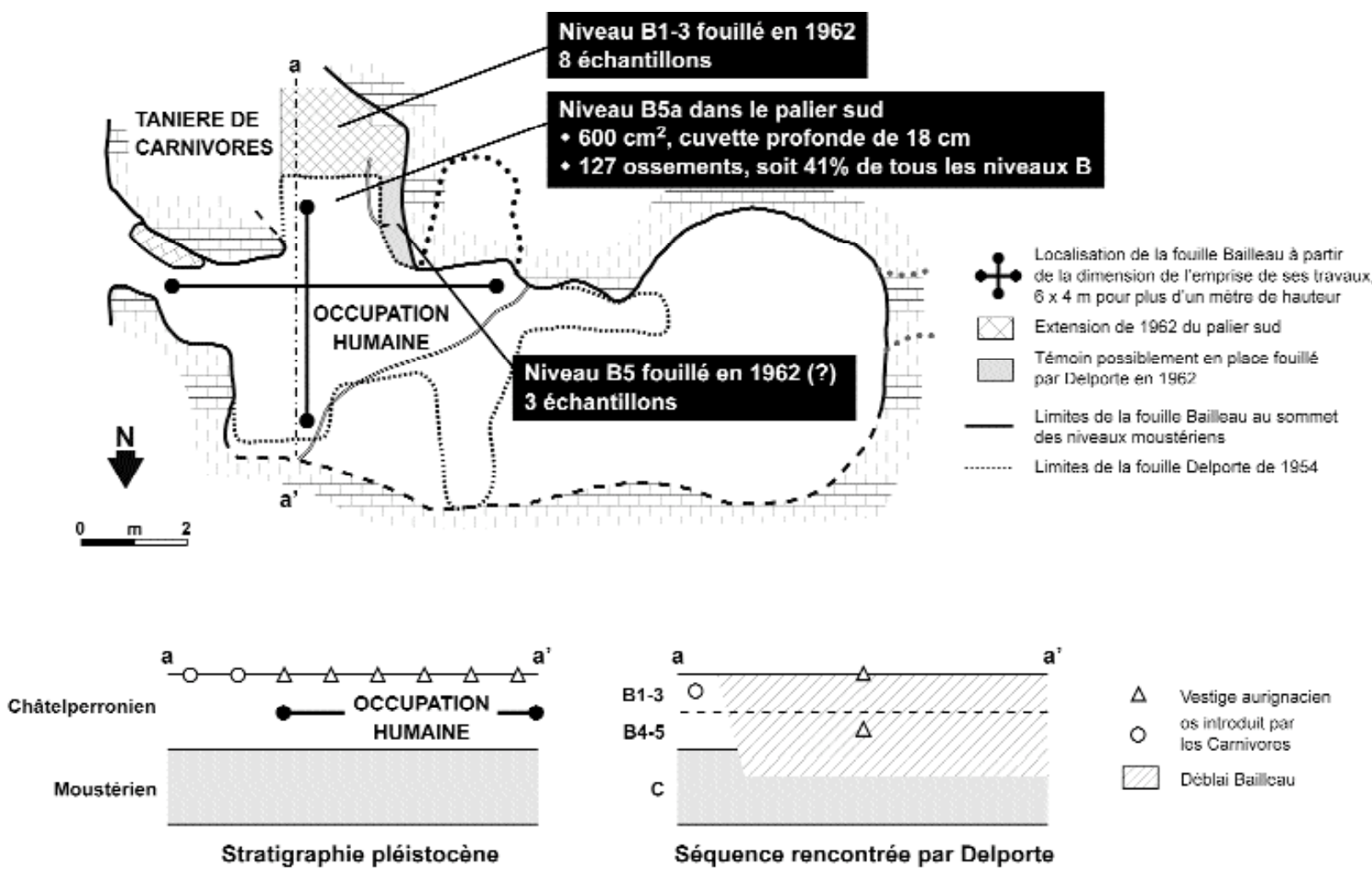

Figure 13 - Provenance des échantillons $14 \mathrm{C}$ de la grotte des Fées portée sur un plan indiquant la nature des dépôts rencontrés. La cohérence des datations est d'abord liée à la structuration spatiale du site : les échantillons du niveau B5 proviennent probablement d'un témoin in situ préservé en périphérie de l'habitat châtelperronien, tandis que les échantillons des niveaux B1-B3 sont issus d'une tanière de carnivores postérieure au Châtelperronien et située dans un renfoncement de la paroi sud de la grotte.

Figure 13 - Radiocarbon sample provenience and horizontal stratigraphy at the Grotte de Fées; since the B5 samples probably come from an in situ remnant in the periphery of the Châtelperronian habitat, and the B1-3 samples from a postChâtelperronian carnivoreden in a recess of the south wall of the cave, consistency in dating relates to spatial patterning.

Le travail que nous avons mené (Zilhão et al. 2006 : 1264412645) établit que la série faunique conservée au Musée des Antiquités Nationales (MAN) rassemble principalement des os de camivores ou accumulés par eux (fig. 11), et donc, de ce point de vue, ne diffère pas de la série de B1-3. Nous en avons déduit que les os datés avaient été accumulés de la même façon. On doit, cependant, tenir compte de la description du site par Bailleau (fig. 8), qui implique que les niveaux châtelperroniens ont contenu une quantité significative de vestiges fauniques d'origine anthropique. Les quelques os brûlés présents dans la collection du MAN témoignent de cette composante. Notre incapacité à reconnaître un signal anthropique plus important est, très probablement, liée au fait que $93 \%$ de la série osseuse des niveaux $B$ regroupés ont été collectés dans deux horizons seulement (tabl. 3). Cette distribution reflète, comme nous le discuterons ci-après, des activités de camivores bien circonscrites dans l'espace. La série osseuse n'offre donc pas une image fidèle de la faune que Bailleau a rencontrée dans la zone centrale à occupations humaines.
Selon cette hypothèse, la cohérence des datations du niveau B5 est facile à comprendre. Qu'elles datent des dépôts in situ n'est pas un point de discorde. Puisque ces vestiges ont été recueillis en 1962, ils sont probablement issus d'un témoin préservé, en retrait de la section ouest et en marge de la concentration fouillée par Bailleau, soit dans un secteur où l'on peut s'attendre à trouver non seulement des objets châtelperroniens, mais également des ossements accumulés par les Châtelperroniens ou par des agents naturels contemporains (fig. 13). Mais dans ce secteur, deux hypothèses sont à considérer : d'une part celle où, pendant le Châtelperronien, aucun reste de faune n'a été accumulé dans le prolongement du palier sud utilisé par les camivores pour leur tanière et, d'autre part, celle où de tels restes ont été accumulés en nombre suffisamment faible pour ne former qu'une fraction minime du palimpseste qui a pu être créé par une activité notable de ces mêmes camivores postérieurement à l'occupation châtelpemonienne. II est alors aisé d'estimer qu'un échantillonnage aléatoire offre une probabilité de sélection d'un os 
d'âge châtelperronien bien inférieure à 1/8. Dans ce cas, ne pas obtenir une date châtelperronienne dans le niveau B1-3 est tout à fait en accord avec notre modèle. II en est de même de la probabilité que les datations révèlent la présence d'ossements d'âge moustérien (cas de l'échantillon daté à plus de 53900 BP, cf. tabl. 4). L'explication avancée par Mellars et alii (2007 : 3660) pour cette " anomalie " (erreur de marquage, mélange par inadvertance au cours de la fouille ou au cours de la manipulation de la collection au Musée) n'est pas seulement un argument ad hoc. Elle est également inutile.

Mellars et collaborateurs n'ont pas tenu compte de cette probabilité dans leur raisonnement. S'ils l'avaient fait, ils auraient évité l'erreur qui consiste à croire que, pour être correcte, notre interprétation nécessiterait l'obtention de dates autour de 39-40 ka ${ }^{14} \mathrm{C}$ BP pour du matériel prove nant, à l'origine, de dépôts sous-jacents, mais trouvés dans B1-3. Comme le montre le tableau 3, le niveau B4-5 est très pauvre. II contient seulement $3 \%$ du total de vestiges recueillis dans l'ensemble des niveaux B et, surtout, aucun ossement. Nous ne doutons pas que Mellars et al. s'accorderont avec nous pour admettre que la probabilité qu'un os soit issu d'un dépôt qui n'en contient pas, ne peut être qu'infinitésimale.

Gravina et al. (2005) ont évidemment pensé que leur procédure d'échantillonnage confirmait, par les datations ${ }^{14} \mathrm{C}$, la solidité du schéma stratigraphique de Delporte et ont admis que la cohérence de 10 des 13 échantillons traités validait cette démarche. En réalité, ces dix échantillons sont issus de deux unités stratigraphiques seulement (B5B5a et B1-3). Pour l'un d'eux, dont l'année de fouille est 1962 , on peut admettre un certain degré de fiabilité du fait de la localisation des échantillons et de l'intégrité des dépôts associés. Les résultats obtenus sont en accord avec cette interprétation mais ils ne démontrent pas l'intégrité des autres unités stratigraphiques. A l'inverse de Gravina et al. ou de Mellars et collaborateurs, nous prétendons que la cohérence de ces dix dates ne traduit pas la fiabilité de l'interprétation stratigraphique de Delporte, mais reflète la répartition en mosaïque (et la dérive chronologique latérale qu'elle implique) des occupations du site par les camivores et les hommes.

Finalement, alors qu'ils représentent moins de $10 \%$ de la surface fouillée par Delporte (cf. fig. 1-3), les deux séries d'échantillons apportant des résultats satisfaisants (B5 et B1-3) correspondent à $93 \%$ de la collection d'ossements issus des niveaux $B$ de la grotte des Fées conservée au MAN (tabl. 3).

\section{5 - INDUSTRIE LITHIQUE}

Mellars et collaborateurs contestent les conclusions de notre étude de la série lithique en discutant sept points de notre argumentation que nous allons développer dans ce qui suit.

\section{1 - Etats de surface}

"La raison pour laquelle la fréquence bien plus importante des pièces présentant une surface altérée dans les niveaux supérieurs que dans les niveaux sous-jacents, est un argument qui soutient l'interprétation de déblais ne nous apparait pas claire. Selon cette hypothèse, toutes les pièces seraient issues directement du stock châtelpenonien des niveaux B4 et B5 sous-jacents (beaucoup moins patinés), et auraient été secondairement rejetées au dessus des niveaux B4 et B5 in situ au cours des fouilles $d u X I X^{e}$ siècle, soit seulement 80 ans avant la fouille Delporte. Comment les vestiges lithiques des niveaux B1-3 peuvent avoir une surface qui s'est significativement dégradée dans les 80 ans qui séparent les fouilles des années 1870 de celles réalisées par Delporte dans les années 1950 reste inexpliqué. (...) dans l'un ou l'autre cas, la plus grande altération de la surface des pièces des niveaux supérieurs s'oppose fortement à un remaniement de ces pièces depuis les niveaux immédiatement sous-jacents, c'est-à-dire les niveaux châtelperroniens de base. " ${ }^{9}$ (p. 3658-3659)

Quel que soit l'emplacement de dépôts intacts couverts de déblais, le seul endroit d'où le matériel archéologique découvert dans les déblais ne peut pas provenir est précisément des niveaux intacts " immédiatement sousjacents ". Si c'était le cas, ces niveaux “ immédiatement sous-jacents " ne pourraient être intacts puisqu'ils auraient été remaniés par la fouille des dépôts sus-jacents. Prenant l'exemple du palier sud, notre interprétation implique que la plupart des objets recueillis dans les niveaux B1 à B3 proviennent d'un autre emplacement du site et qu'ils ont été transportés puis finalement abandonnés là où Delporte les a trouvés. Si les pièces recueillies dans ces niveaux sont plus patinées, cela peut indiquer un contexte géochimique différent de celui d'origine.

Toutefois, en confondant " une fréquence plus importante de pièces présentant une 'surface altérée' " avec " une surface qui s'est significativement dégradée " en " 80 ans", Mellars et alii ne font que malmener le concept élémentaire de variation de fréquence. Les couches B1 à B3 livrent un nombre plus important de pièces patinées ou ébréchées que les couches

(9) 'Why the much higher frequency of 'surface weathered' pieces in the upper as opposed to lower levels should favor the backdirt interpretation is again unclear to us, because according to the backdirt model all of these pieces must derive directly from the underlying (much less patinated) Chatelperronian material in levels B4 and B5, which were subsequently dumped on top of the in situ B4 and B5 levels in the course of the 19th century excavations, only 80 years before the time of Delporte's excavations. How the lithic artefacts in levels B1-B3 could have acquired this greatly increased 'surface alteration' in the 80 years between the 1870s excavations and those of Delporte in the 1950s remains unexplained. (...) in either event, the increased surface weathering of the pieces in the upper levels is strongly opposed to the recent backdirt derivation of these pieces from the immediately underlying, basal Chatelperronian levels." 

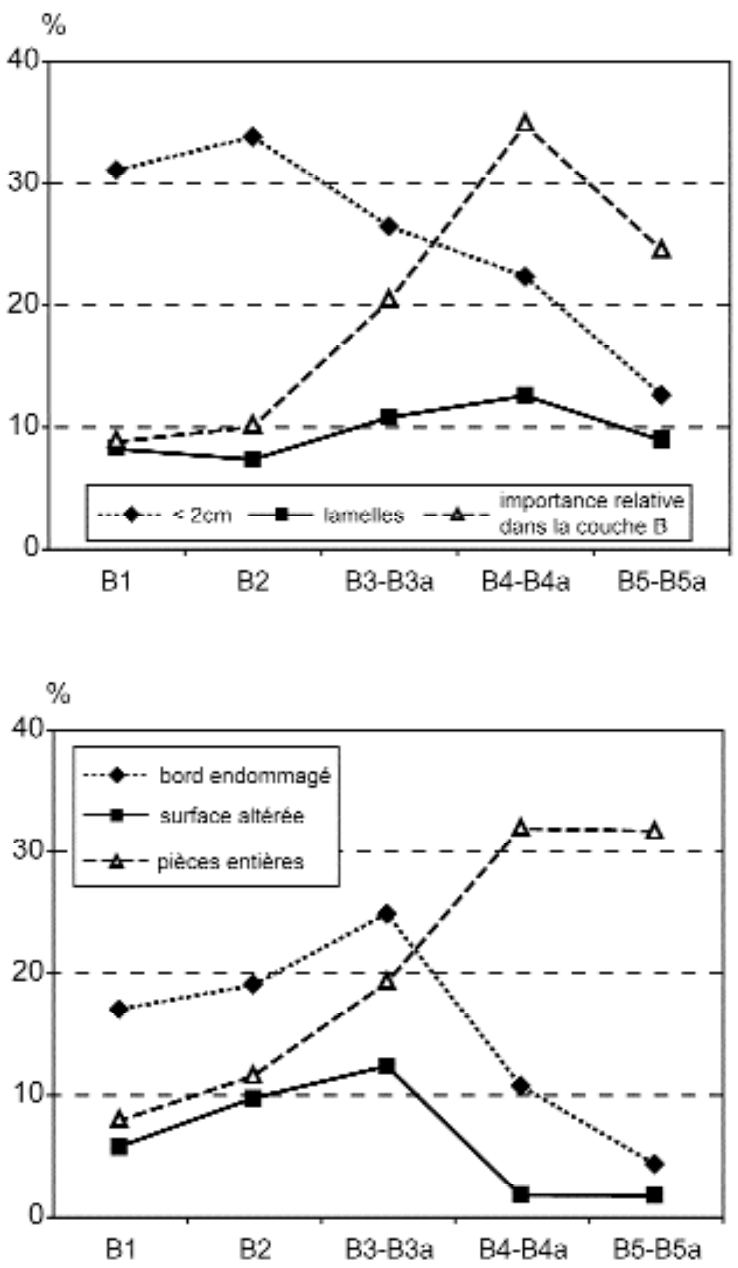

Figure 14 - Evolution, sur l'ensemble des niveaux BB, de la valeur des paramètres taphonomiques pris en compte pour l'examen de la série lithique. En haut : fréquence relative des lamelles et des petits vestiges $(<2 \mathrm{~cm})$ et proportion de la masse d'objets de chaque niveau rapporté à la totalité du matériel récolté dans l'ensemble $B$. En bas : fréquence relative des bords endommagés, d'altération de surface et de pièces entières (esquilles et cassons exclus), selon Zilhão et alii (2006 : fig. 3).

Figure 14 - Lithic indicators of taphonomic process in the $B$ levels. Up: relative frequencies per level of bladelets and items $<2 \mathrm{~cm}$, and percentage of the overall $B$ finds represented by each level. Down: relative frequencies per level of edge-damaged, surface-weathered and unbroken pieces (chips and chunks excluded) (after Zilhão et al. 2006: Figure 3).
B4-B5. Mais les pièces des niveaux B1à B3 ne sont pas plus altérées que celles de B4-B5 (fig. 14). On peut supposer que les premiers fouilleurs auraient préféré délaisser ou rejeter les éléments de petite taille, fragmentés ou endommagés (trouvés dans les déblais par les nouvelles générations de chercheurs) tandis que les pièces entières ou de plus grande taille auraient été recueillies. Les données de la grotte des Fées, notamment le contraste existant entre les niveaux $\mathrm{B} 1$ à $\mathrm{B} 3$, d'une part, et B4-B5, d'autre part, s'accordent avec cette possibilité et soutiennent donc notre interprétation.

\section{2 - Objets retouchés et cassés}

Mellars et ses collaborateurs se demandent " pourquoi d'aussi nombreuses pièces retouchées ont été ignorées au cours des fouilles $d u 19^{\circ}$ siècle et se sont ainsi retrouvées dans les déblais " ${ }^{10}$ et "que recherchaient exactement les fouilleurs $d u$ $19 e$ siècle si ce n'est l'outillage en silex ? "11 (p. 3660)

Comme permet de le prévoir notre interprétation, les " nombreuses pièces retouchées " issues des niveaux B1 à B3 de Delporte sont pour la plupart petites. Elles consistent souvent en fragments atypiques que l'archéologue actuel reconnaît mais que le collectionneur de fossiles du XIX ${ }^{e}$ siècle laissait volontiers partir au rebut, tout comme les produits de débitage (fig. 15). En réalité, les niveaux B1 et B2 n'ont pas livré à Delporte une seule pointe de Châtelperron. La pièce qui s'en rapproche le plus (encadrée dans la partie inférieure de la fig. 15) a ainsi été décrite comme " une forme de lame à dos peu typique " car " il n'y a pas d'abatage caractérisé, mais simplement accommodation de l'arête par une retouche incomplète et peu abrupte " (Delporte 1957 : 466-467; $n^{\circ} 31$ de la planche Vl). Par comparaison, les nombreuses pointes de Châtelperron que Lacaille (1947) représente dans sa publication des collections du XIX ${ }^{\ominus}$ siècle de la grotte des Fées sont complètes et typiques (fig. 16).

Parmi la série du niveau B2, une pièce présente une cassure récente qui semble être le fait d'un outil de métal et que recouvre un film de sédiment en partie induré (fig. 17) qui implique que la pièce a été cassée puis enfouie jusqu'à ce que Delporte la découvre. II s'agit là d'une preuve matérielle que les niveaux $\mathrm{B} 1$ à $\mathrm{B} 3$ contiennent des éléments rejetés au XIX ${ }^{e}$ siècle par les fouilleurs de la grotte des Fées.

\section{3 - Pièce solutréenne du niveau B2}

"En ce qui concerne le fragment de pièce solutréenne, nous notons que Delporte n'en a jamais fait mention ou seulement illustré cette pièce lors de la méticuleuse analyse du matériel lithique (...) et que Zilhão et al. ne fournissent aucune illustration de cette pièce soi-disant cruciale dans leur publication " 12 (p. 3660).

(10) "why so many retouched pieces were overlooked during the 19th century excavations only to find their way into the discarded backdirt deposits"

(11) "what exactly were the 19th century excavators looking for in their excavations, if not retouched stone tools?"

(12) "As regards the fragmentary Solutrian piece, we note that Delporte never mentioned or illustrated this piece in his own meticulous analysis of the lithic material (...) and that Zilhão et al. provide no illustration of this supposedly crucial piece in their paper". 

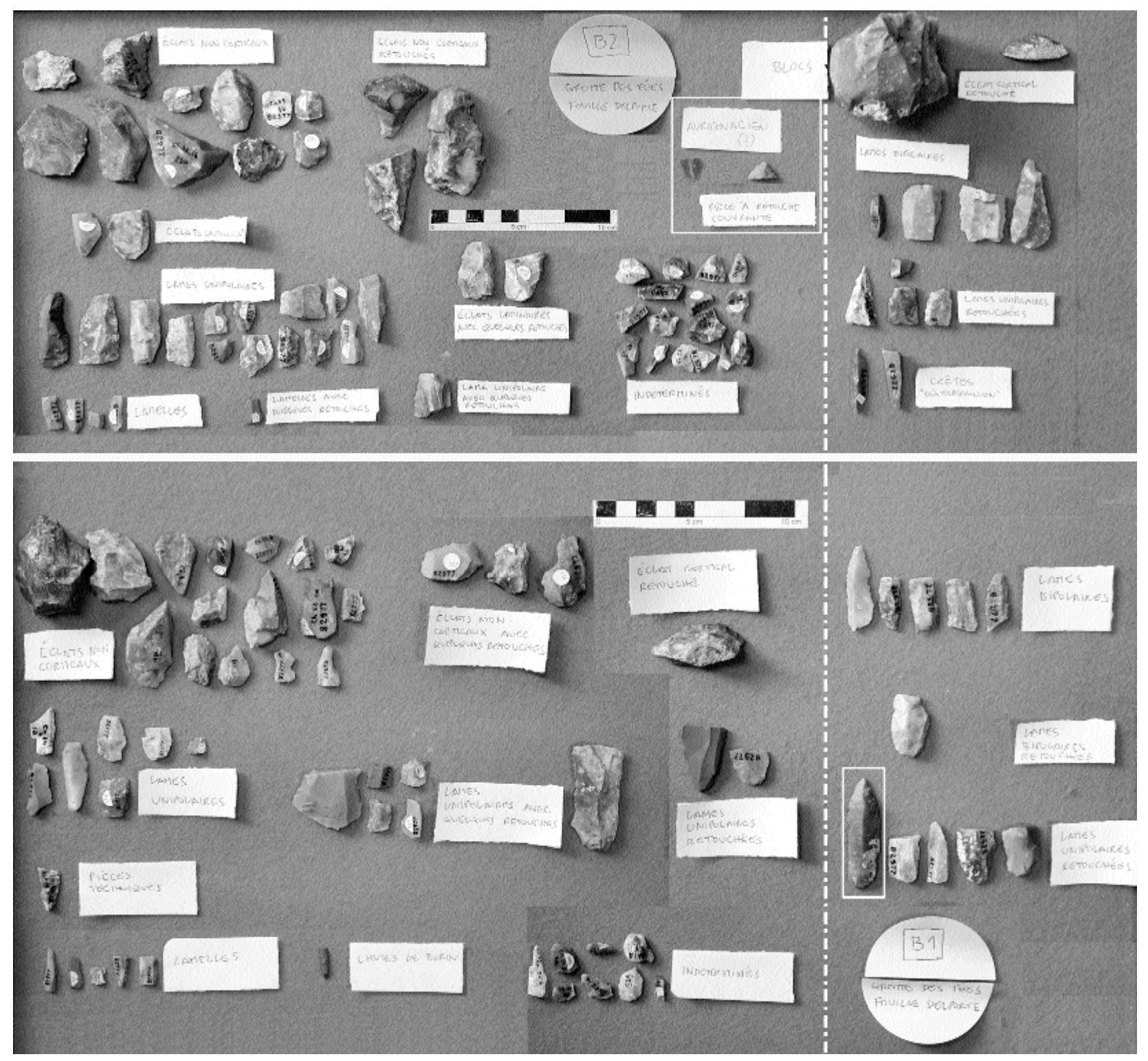

Figure 15 - Vue des séries lithiques des niveaux B1 (en bas) et B2 (en haut) à partir de photos prises au MAN en novembre 2005 lors de l'examen des collections. Ces séries offrent de bons exemples du type de matériel que des fouilleurs du XIXe siècle pourraient avoir laisser échapper et que Delporte aurait pu recueillir en fouillant leurs déblais. Le matériel qui présente des caractères diagnostiques châtelperroniens est placé à droite de la ligne pointillée. La pièce encadrée dans la série B1 est l'unique pointe de Châtelperron, atypique, rencontrée dans ces deux niveaux. Les deux pièces encadrées de la série B2 sont deux éléments de petite taille, mais typiquement aurignacien pour l'un et solutréen pour l'autre (cf. fig. 18, 20b).

Figure 15 - Overall view of the lithic assemblages from levels B1 (bottom) and B2 (top) of the Grotte des Fées, assembled from photographs taken at the MAN in November 2005 as the material was being laid out. These assemblages are good examples of the kind of material that 19th-century excavators would have discarded and that Delporte would have collected when reexcavating their backfill. The Châtelperronian diagnostic material is that right of the vertical dashed white line; the framed piece in B1 indicates the single, atypical Châtelperron point found in these two units; the framed area in B2 isolates two small pieces, one possibly Solutrean, the other diagnostically Aurignacian (cf. fig. 18, 20-b). 


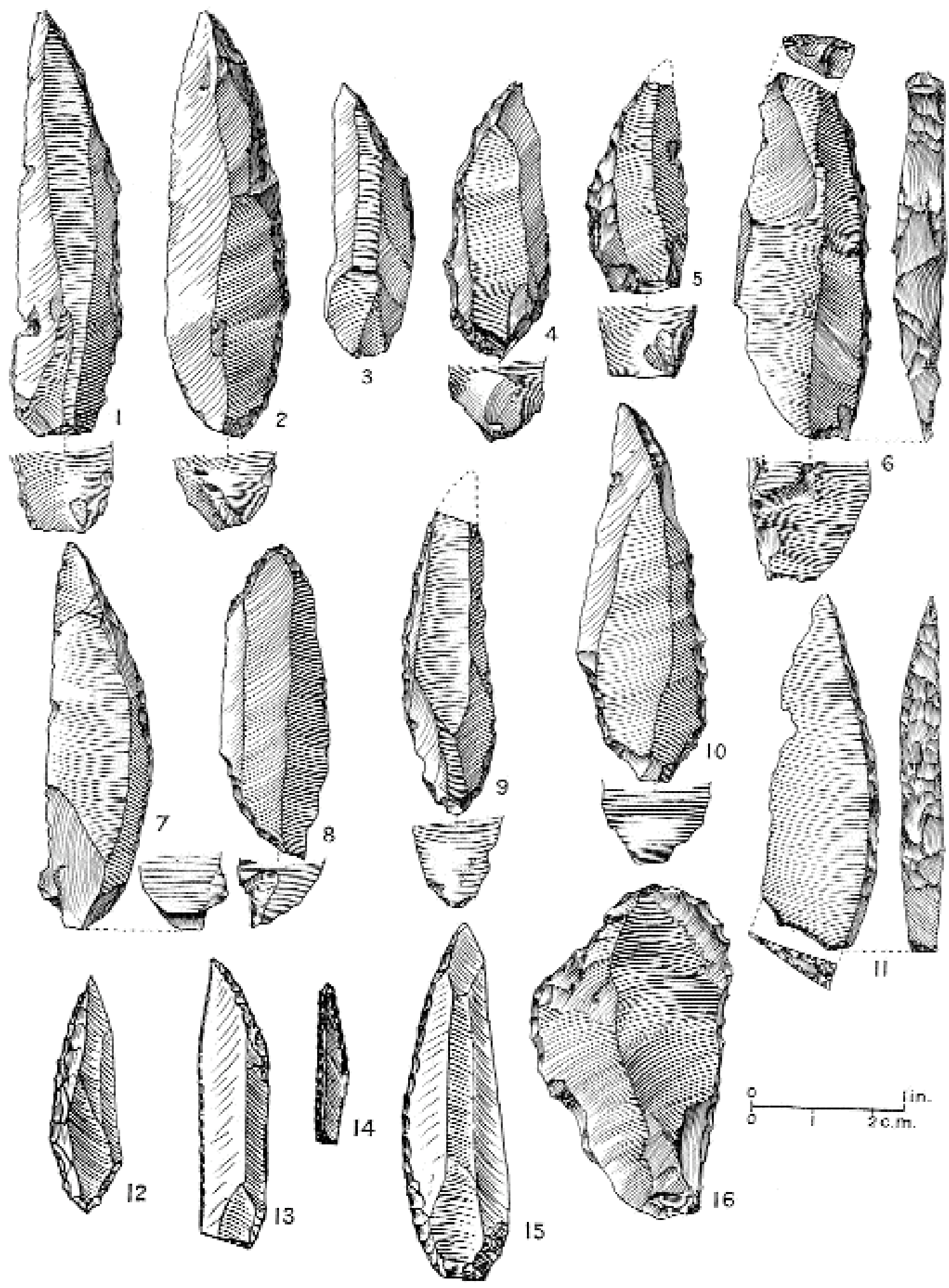

Figure 16 - Outils de silex à dos abrupt (" steeply dressed flint implements ") provenant de Châtelperron et illustrés par Lacaille (1947 : fig. 2).

Figure 16 - Lacaille's (1947: Figure 2) illustration of "steeply dressed flint implements" from Châtelperron. 


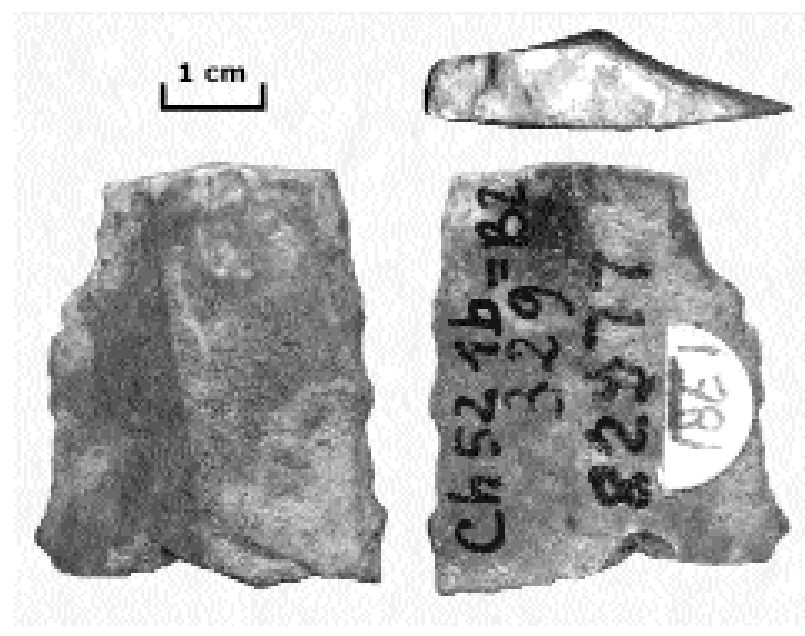

Le marquage de la pièce en question (fig. 18) ne souffre aucune ambiguitté. Elle a été illustrée dans l'inventaire de la collection de B. Depraetere (2000), un travail réalisé avec l'assistance de Delporte lui-même. Bien que petit, ce fragment est entièrement couvert, sur la face dorsale, d'une retouche plate et envahissante qui caractérise le style solutréen (Smith 1966 ; Tixier et al. 1980 ; Demars et Laurent 1989 : fig. 51 ; Inizan et al. 1992). D'après notre expérience acquise par la manipulation et l'examen de centaines de milliers de pièces du Paléolithique supérieur d'Europe occidentale, il semblerait que ce mode de retouche ne soit pas connu au Châtelperronien. Enfin, tandis que toutes les pièces typiquement châtelperroniennes sont exclusivement confectionnées dans un silex local, cette pièce est taillée dans un matériau allochtone dont les affleurements connus se situent dans le Bassin parisien. II s'agit d'un silex blond à grain fin, translucide et homogène ne contenant que de très rares fossiles marins turoniens (Aubry 1991 ; Depraetere 2000).

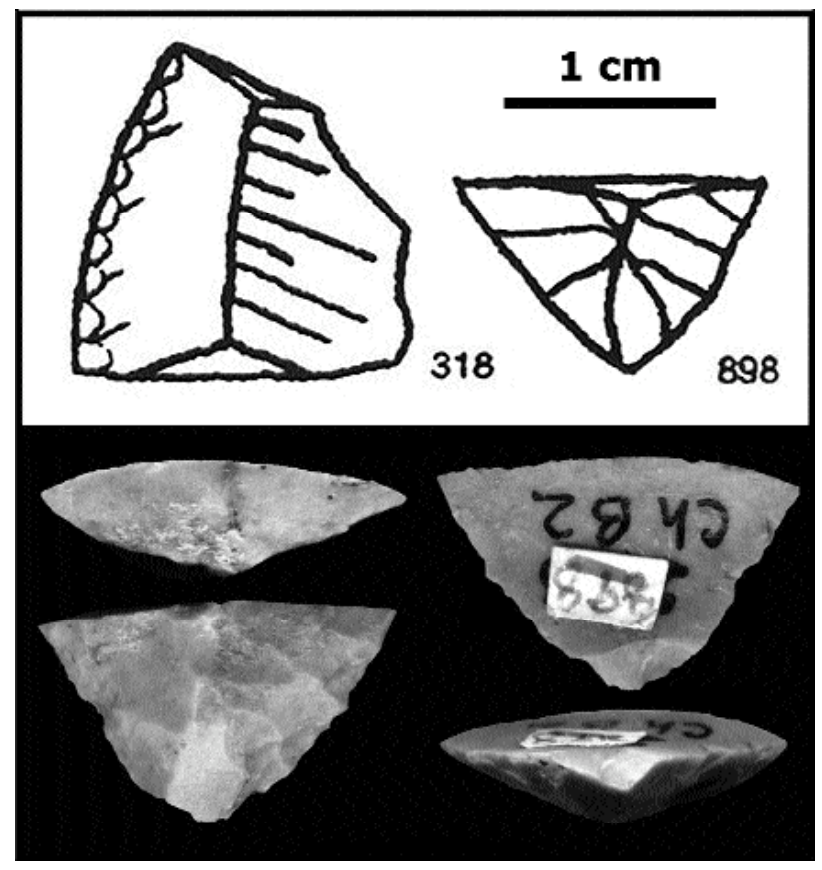

Figure 17 - Pièce de silex présentant une cassure fraîche couverte d'un film de sédiment partiellement induré. Cet objet est issu du niveau B2 de Delporte, montrant par là même que ces niveaux correspondent à des déblais $d u$ XIXe siècle.

Figure 17 - A flint piece with a fresh break covered by a semi-concreted sediment film from Delporte level B2 provides corroborating evidence that these levels correspond indeed to 19th-century backfill.
Ce type de retouche se rencontre parfois en partie distale d'outils provenant d'autres technocomplexes, à savoir le Gravettien (Klaric 2003 : fig. 165), voire le Magdalénien (Demars and Laurent 1989 : fig. 11, nos. 4-5 ; fig. 27, no. 5). Sur un plan théorique, on peut même envisager la possibilité que cette retouche envahissante soit produite par le " débordement " d'une retouche scalariforme, en particulier si cette dernière forme de retouche typique des industries aurignaciennes est appliquée à l'extrémité d'une lame épaisse à face dorsale convexe, comme cela est le cas pour cet objet. Une attribution au Gravettien ou à l'Aurignacien, bien que plus hasardeuse, ne saurait être tout à fait exclue. Néanmoins, cette pièce témoigne que le niveau B2 n'a pas livré une série châtelperronienne homogène, et si l'attribution à l'Aurignacien devait se confirmer, ce serait là une indication supplémentaire que de telles pièces sont éparpillées dans l'ensemble de la séquence Delporte, et non pas regroupées au sein d'une même lentille (cf. infra).

Figure 18 - En haut: "Lames retouchées " de la grotte de Fées, d'après Depraetere (2000 : fig. 34). Cet auteur a décrit le $n^{\circ} 318$ comme "une lame à retouche abrupte "et le $n^{\circ} 898$ comme une "lame à large retouche ". En bas : différentes vues de la pièce $n^{\circ} 898$ montrant la retouche envahissante fréquemment rencontrée au Solutréen et inconnue dans les industries châtelperroniennes.

Figure 18 - Above: the "retouched blades" from the Grotte des Fées, after Depraetere (2000: Figure 34); she describes no. 318 as a "blade with abrupt retouch" and no. 898 as a "blade with large retouche". Below: different views of object no. 898, showing the kind of flat, invasive retouch commonly found in the Solutrean and unknown in the Châtelperronian. 


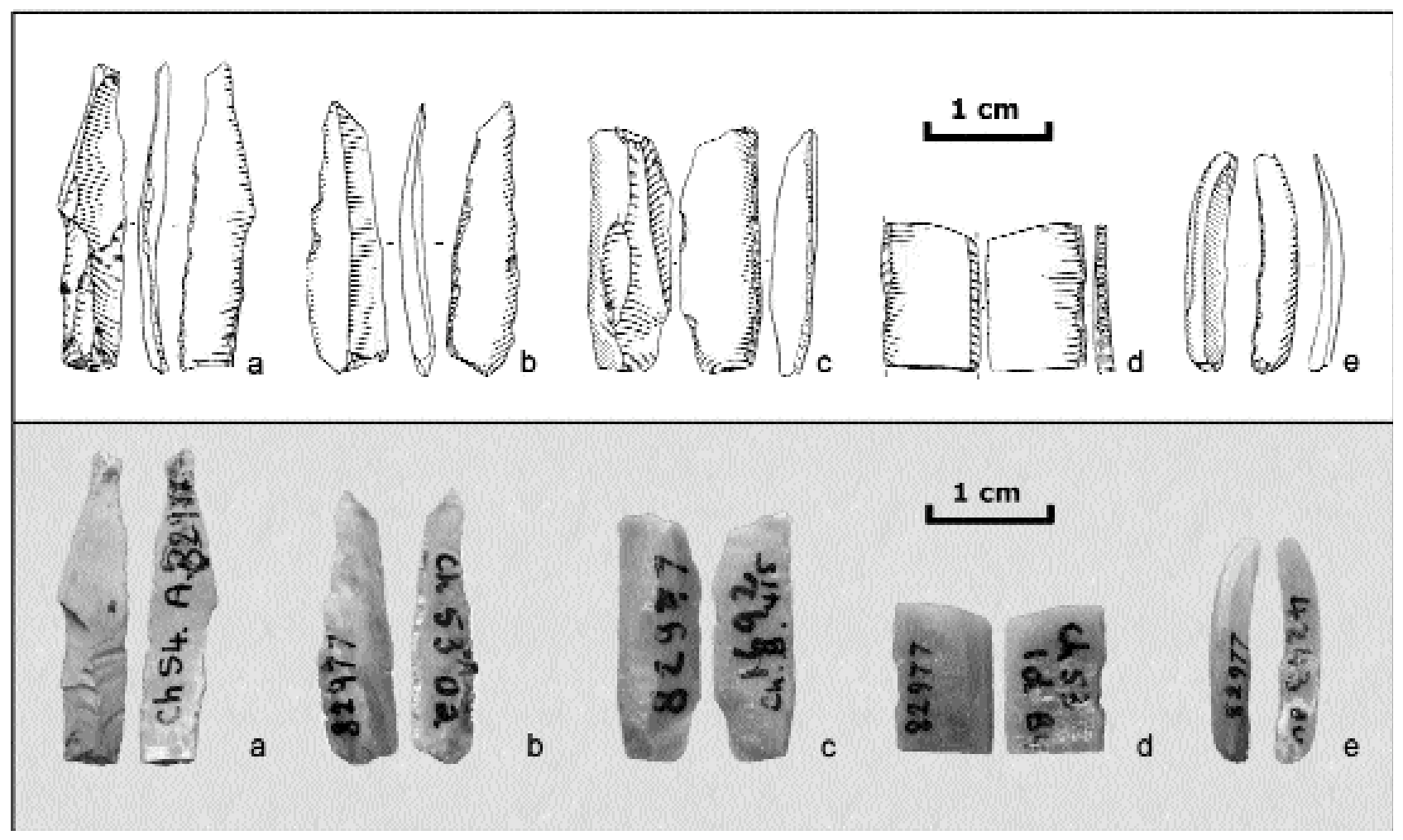

Figure 19 - Lamelles Dufour de la grotte des Fées: a-b, niveau A (a. 1954 ; b. 1953) ; c. niveau B4-5 (1962) ; d-e. niveau B4 (d. 1953; e. 1954).

Fiure 19 - Dufour bladelets from the grotte des Fées: a-b. level A (a. 1954 ; b. 1953) ; c. level B4-5 (1962); d-e. level B4 (d. 1953 ; e. 1954).

\section{4 - Typologie des lamelles Dufour}

"Nous sommes également sceptique vis-à-vis de leur interprétation des petites lamelles Dufour issues du niveau B4 comme étant des pièces diagnostiques d'un Aurignacien II "13 (p. 3660).

Nous maintenons ce point de vue mais, du fait des nombreuses opinions qui existent sur ce sujet parmi les auteurs, les différences d'appréciation de la valeur chronostratigraphique des lamelles Dufour ne sauraient être débattues dans le cadre de cet article. Nous remarquons, cependant, que Mellars et al. (p. 3661) considèrent comme " particulièrement typiques " 14 et indiquant un " bref épisode d'occupation par des groupes aurignaciens plus tardifs " ${ }^{15}$ les deux lamelles Dufour recueillies dans la couche humifère (couche A) (fig. $19 a-b)$. Eu égard à la provenance des pièces, une telle inter- prétation chronologique ne peut se baser que sur des considérations typologiques. Toutefois, la lamelle Dufour issue des niveaux B4-5 de la fouille de 1962 (fig. 19c) étant, en tout point, identique à celle de la couche $A$, nous nous rangeons à l'avis expert de Mellars et alii pour reconnaître que des pièces signant un "Aurignacien plus tardif" sont présentes au sein des horizons châtelperroniens, comme nous l'avions d'abord indiqué sur la base de la lamelle recueillie en B4.

\section{5 - Distribution des vestiges aurignaciens}

“Que des pièces diagnostiques d'un Aurignacien aient été trouvées dans un autre niveau [i.e. autre que B4] de la séquence châtelperronienne nous parait particulièrement douteux. Nous ne sommes franchement pas convaincus par le fragment ténu $(\sim 2 \mathrm{~cm})$ d'un soi-disant "grattoir en bout de lame aurignacienne " provenant du niveau B2 "16 (p. 3661)

(13) "We are equally unconvinced by their interpretation of the small, retouched Dufour bladelet from level B4 as a diagnostically 'Aurignacian II' artifact'.

(14) "highly typical"

(15) "brief episode of occupation by later Aurignacian groups"

(16) "Whether any diagnostically Aurignacian pieces were found in any other levels [i.e., other than B4] of the Chatelperronian sequence appears to us open to serious doubt. We are frankly unconvinced by the tiny $(22 \mathrm{~cm})$ fragment of a supposedly 'end scraper on Aurignacian blade' recovered from level B2". 


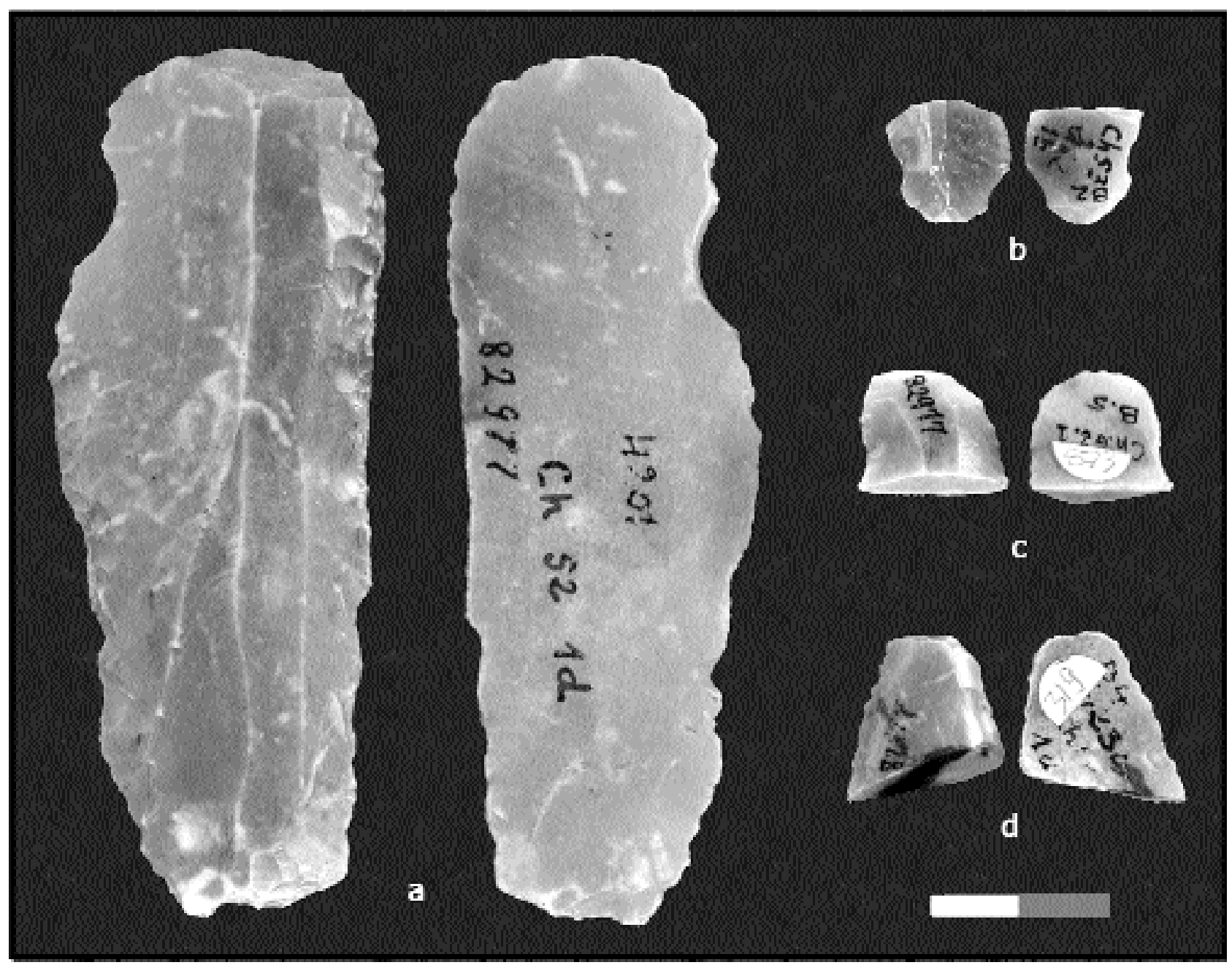

Figure 20 - Pièces retouchées en silex allochtone qui, à la grotte des Fées, n'est rencontré que dans le cas de pièces aurignaciennes typiques. A gauche, grattoir double sur lame retouchée présentant une retouche typiquement aurignacienne, niveau $B 4$ (1952). A droite : fragments d'outils réalisés dans la même matière première et présentant le même type de retouche : $b$. niveau B2, fouille 1953 : c. niveau B5, fouille 1962 ; d. niveau B4, fouille 1952.

Figure 20 - Retouched tools made on an exotic flint raw-material that, in the Grotte des Fées, was exclusively used for diagnostic Aurignacian pieces: a. endscraper with characteristic Aurignacian edge retouch, typical of the Aurignacian I, from B4 (1952); b.-d. small tool (possibly endscraper) fragments with the same kind of edge retouch and made in the same raw-material (b. from B2, 1953; c. from B5, 1962; d. from B4, 1952).

"Nous concluons (...) qu'il existait une concentration manifeste de pièces typiquement aurignaciennes au sein ou à proximité immédiate du niveau B4 de sa stratigraphie [celle de Delporte], clairement stratifié entre le matériel typiquement châtelperronien de son niveau B5 et les séries aussi typiquement châtelperroniennes des niveaux supérieurs B1-B3" 17 (p. 3661-3662).

La pièce provenant du niveau B2 n'est pas plus petite que les deux autres fragments présents en B4 et B5 que
Mellars et al. considèrent comme diagnostiques et qui ont précédemment été représentées par ces mêmes auteurs (Gravina et al. $2005: n^{\circ} 6$ et 8 de la fig. 2, légendées "lames aurignaciennes à bord retouché "). La question de la dimension mise à part, la pièce soi-disant peu convaincante, présente une retouche identique à celle des deux autres objets. En outre, cette pièce du niveau B2 est taillée dans le même silex " allochtone, de bonne qualité et aisément identifiable" ${ }^{18}$ qui, selon

(17) "We conclude that (...) there was a clear concentration of diagnostically Aurignacian artefacts within or immediately adjacent to level B4 of his stratigraphy, clearly stratified between the typically Chatelperronian material in his level B5 and the equally typical Chatelperronian material in the overlying levels B1-B3".

(18) "distinctive, high quality imported". 


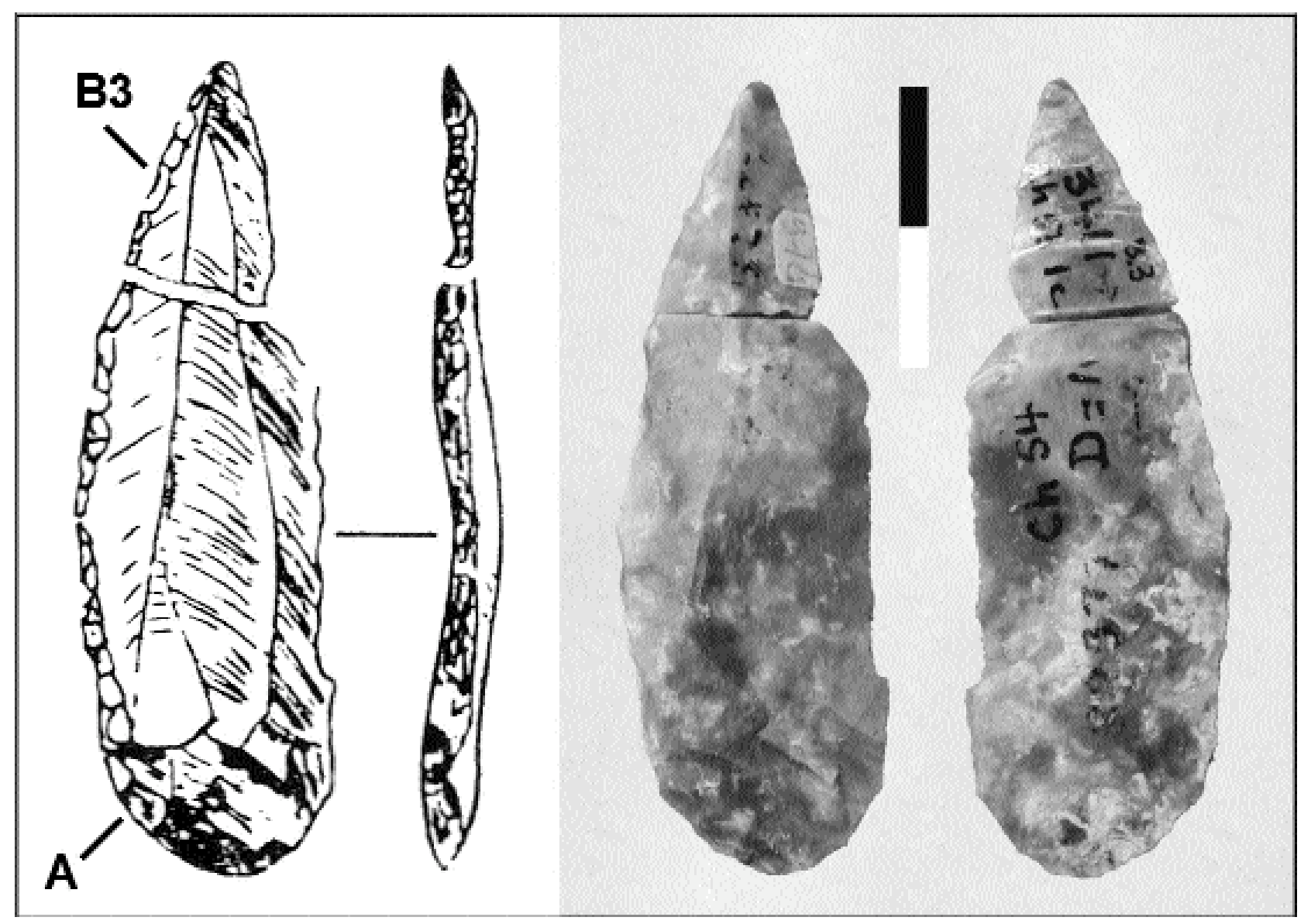

Figure 21 - Pointe de Châtelperron raccordée à partir de deux fragments. Le fragment apical est issu du niveau B3 et le fragment basal a été recueilli dans la couche humifère (niveau A). Dessin d’après Delporte (1957 : pl. VI, n²6, modifié).

Figure 21 - Châtelperron point refitted by Delporte from two fragments, the tip in level B3 and the base in the disturbed topsoil (level A). Drawing after Delporte, 1957 (PI. VI, no. 26, modified).

Mellars et al., a été utilisé que pour la confection de toutes les autres pièces typiquement aurignaciennes (fig. 20). Cette matière première si particulière est connue dans les vallées du Cher et de l'Indre, distantes d'environ 200 km (Aubry 1991 ; Depraetere 2000). Aucune pièce châtelperronienne ou non diagnostique n'a été façonnée dans cette matière première.

Quelle qu'en soit la raison, il est évident que les vestiges aurignaciens sont disséminés entre le niveau $A$ en sommet de séquence et le niveau B5 à sa base. Lorsqu'on considère l'année de fouille (et par là même le lieu de provenance des pièces), le trait le plus frappant de la concentration du niveau B4-B4a, qui a une si grande portée selon Mellars et collaborateurs, est que, parmi les cinq objets qui la composent, trois sont issus de la fouille de 1952 (cf. fig. $20 \mathrm{a}, \mathrm{d}$; la troisième pièce est un grattoir caréné (Zilhão et al. 2006 : fig. 2c). Lorsqu'on considère les objets de parure, dont il est fort possible que l'un soit aurignacien (cf. infra, fig. 24), la proportion est de quatre pour six. Ainsi, les deux tiers des objets qui forment cette " concentration" proviennent de la tranchée longitudinale dont nous savons qu'elle a recoupé les déblais dans la partie centrale du site fouillée par Bailleau (cf. fig. 3,9).

En bref, l'argument que Mellars et alii considèrent comme le plus pertinent va, de fait, à l'encontre de leur point de vue : la " concentration" en B4-B4a correspond plutôt à l'année 1952. Cela ne prouve aucunement une interstratification mais suggère au contraire, que les pièces proviennent des déblais. En réalité, de tous les éléments aurignaciens recueillis par Delporte, deux seulement peuvent représenter des intrusions post-dépositionelles dans un témoin in situ (le fragment de grattoir du niveau B5 et la lamelle Dufour du niveau B4-5, tous deux collectés en 1962). Les autres, soit sept sur douze (58 \%) dérivent clairement des déblais : deux du niveau $A$ et quatre de la tranchée de 1952. II est également probable que les trois autres pièces proviennent de dépôts remaniés puisqu'elles ont été recueillies toutes trois au cours des fouilles de 1953 et 1954, alors que la plupart, si ce n'est la totalité des dépôts fouillés ces années-là, étaient des déblais. 


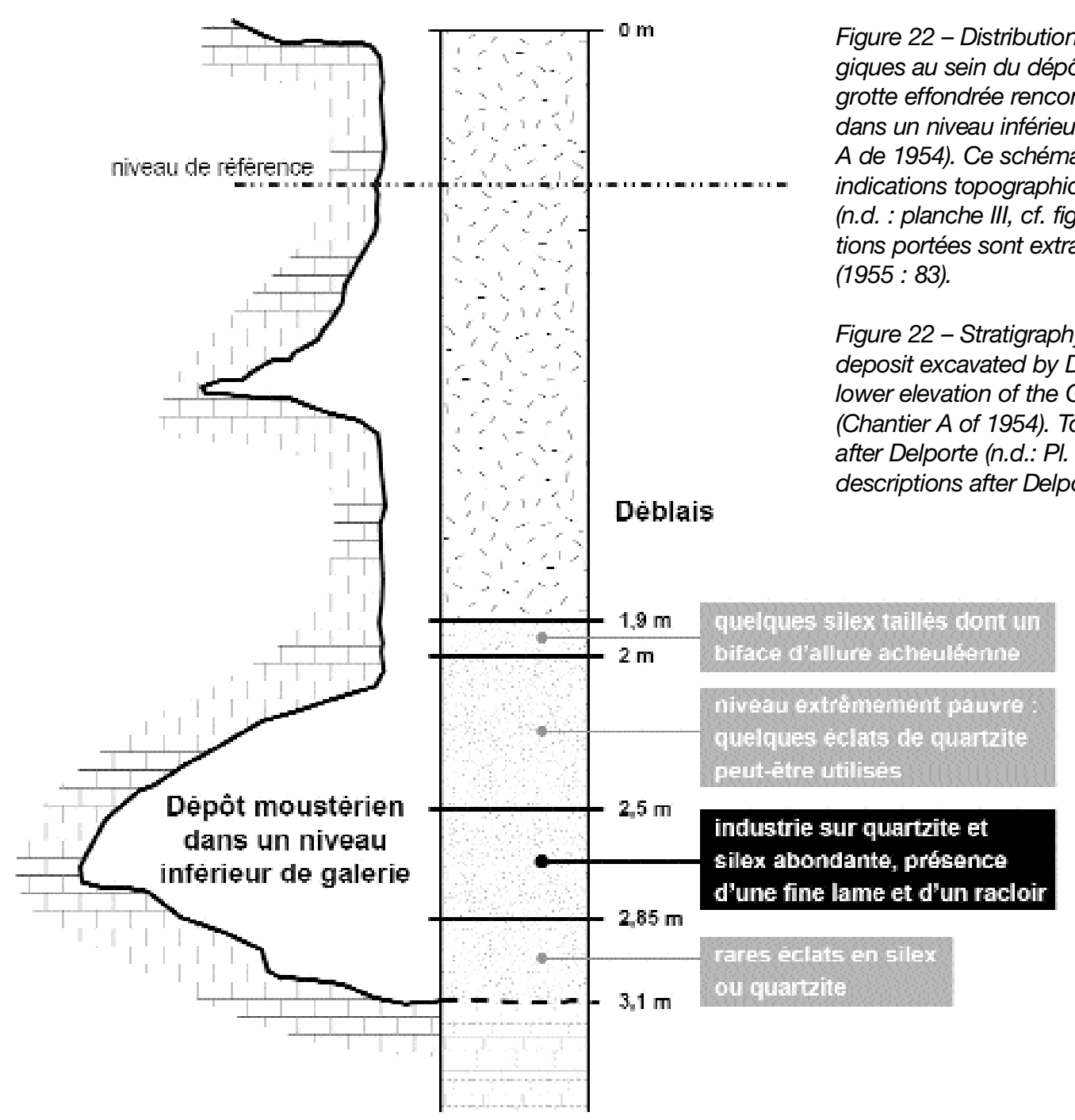

\section{6 - Remontages}

"Il doit également être noté que le seul exemple de raccord au sein de la séquence châtelperronienne a été réalisé par Delporte lui-même (...) entre deux fragments d'une pointe châtelperronienne qui ont été recueillis, respectivement, dans le niveau B3 et dans la couche humifère (niveau 1), confirmant sa conclusion que cet horizon humifère contenait de véritables déblais issus des fouilles pratiquées au $19^{\circ}$ siècle. De façon significative, Zilhão et al. (...) n'ont pas trouvé de remontages entre le matériel des niveaux B4-5 et celui des niveaux B1-B3 qu'ils supposent être des déblais "19 (p. 3661).

Bien que nous ne les ayons pas recherchés systématiquement, d'autres raccords ont été trouvés, par exemple entre deux fragments d'un nucléus en B5a. $\mathrm{Au}$ vu des processus de formation du site reconnus, les chances de succès étaient extrêmement faibles et cette technique ne pouvait en tout cas pas apporter beaucoup à la compréhension de la taphonomie du site. Dans tous les cas, le véritable intérêt de la pièce mentionnée par Mellars et alii, est qu'elle met en relation les niveaux A à B3 par un raccord s'ajoutant à celui qui joint un large fragment proximal issu du niveau $A$ à un fin fragment distal du niveau B3 (fig. 21). Tout cela s'accorde avec l'interprétation que les niveaux $\mathrm{B} 1$ à $\mathrm{B} 3$ sont des déblais et se concilie mal avec l'idée que les niveaux intermédiaires B1 et B2 seraient en place, comme l'impliquerait l'hypothèse de l'interstratification.

(19) "It might also be noted that the only instance of refitting within the Châtelperron sequence was recorded by Delporte himself (...) for two fragments of a Châtelperron point recovered respectively from level B3 and the topsoil horizon (layer A), confirming his conclusion that the topsoil horizon did contain some genuine backdirt from the 19th century excavations. Significantly, Zilhão et al. (...) found no examples of refits between the material from levels B4-B5 and their inferred backdirt in levels B1-B3". 


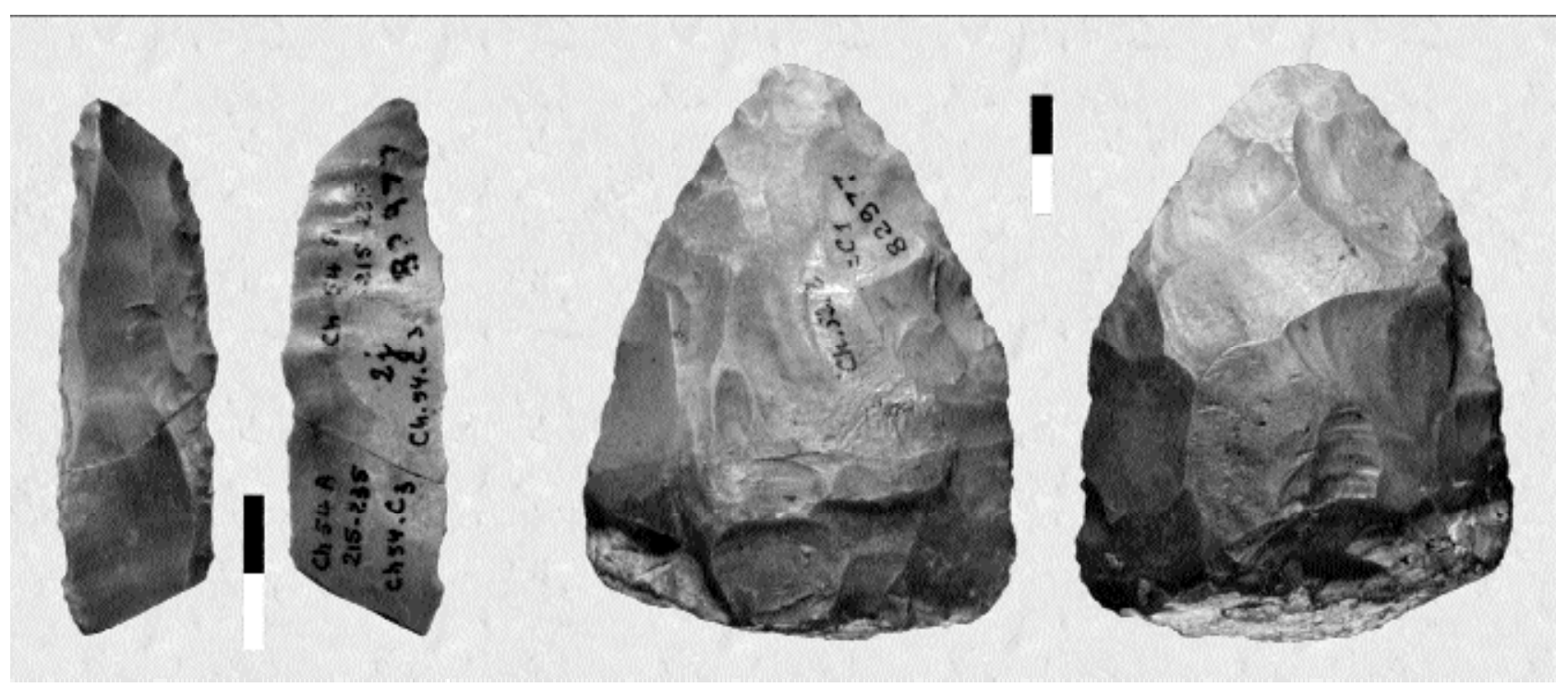

Figure 23 - A gauche : la " fine lame " provenant du principal niveau moustérien (C3) préservé dans le niveau inférieur de galerie et portant la mention " chantier A, 1954 " et une indication de profondeur de "215-235" sous le niveau de base, soit 2,65 à 2,95m. sous la surface du sol. A droite : "biface d'aspect acheuléen" issu du niveau 2, 1952 (couche C1 dans Delporte 1957 $: 457$, planche III, $n^{\circ}$ 2) réputé trouvé à la même profondeur qu'une pièce de 1954 située elle à 1,9 à $2 \mathrm{~m}$ sous la surface du sol (Delporte 1955 : 83).

Figure 23 - Left, the "fine blade" from the main Mousterian level (C3) in the gallery at lower elevation; the marking indicates excavation spit (2j), area/year (Chantier A, 1954), and depth below datum (215-235, i.e., 2.65-2.95 m below ground surface). Right, a "fine biface of Acheulean aspect" from level 2, 1952 (level C1 in Delporte 1957: 457; PI. III, no. 2), reportedly found at the same depth as a 1954 piece from 1.90-2.00 m below ground surface (Delporte 1955: 83).

\section{7 - Présence de vestiges moustériens}

"Enfin, nous remarquons qu'aucun des niveaux supérieurs, qui sont soi-disant des déblais (niveaux B1-B3), n'a livré de pièces typiquement moustériennes (...) puisqu'il est bien connu que les niveaux moustériens de base ont été intensément fouillés au cours des travaux pratiqués au $19^{e}$ siècle sur le site ${ }^{20}$ (p. 3661).

L'absence de matériel moustérien dans les niveaux B1 à B3 ne contredit pas notre interprétation.

La proposition de Mellars et alii selon laquelle les niveaux moustériens ont été " intensément fouillés " au XIX ${ }^{\circledR}$ siècle n'est pas fondée. Bailleau a rencontré des niveauX archéologiques et les a fouillés jusqu'à une profondeur d'un peu plus d'un mètre et ce, dans un secteur du gisement situé à proximité de l'entrée. Delporte, quant à lui, a découvert des niveaux moustériens intacts à une profondeur d'environ 1,5 $\mathrm{m}$ en dessous du sol (cf. fig. 8-9). La raison pour laquelle Bailleau n'a pas approfondi sa fouille est facile à comprendre sur la base de la stratigraphie des dépôts moustériens rapportée par Delporte (1955 : 83 ; 1957 : 457). C'est soixante centimètres sous le niveau atteint par
Bailleau, et après avoir traversé des dépôts " pauvres ", voire " presque stériles " que Delporte a rencontré une " abondante industrie". De plus, ces derniers étaient situés dans un niveau inférieur de la galerie qui, selon Delporte, n'aurait pas été vu ou touché par Bailleau (fig. 22 ; cf. Chantier A de la fig. 2(b), ou secteur e. de la fig. 3).

\begin{tabular}{|l|c|c|c|} 
Type & $\begin{array}{c}\text { Collection } \\
\text { Bailleau }\end{array}$ & $\begin{array}{c}\text { fouille Delporte, } \\
\text { niveau C }\end{array}$ & Total \\
\hline Racloir & 3 & 3 & 6 \\
Pointe & - & 3 & 3 \\
Couteau à dos & - & 1 & 1 \\
naturel & - & 1 & 1 \\
Lame rctouchice & 3 & 3 & 6 \\
Biface & 6 & 11 & 17 \\
\hline Total & & & \\
\hline
\end{tabular}

Tableau 5 - décompte de l'outillage issu des niveaux moustériens de la grotte des Fées, selon Bailleau (1872) et Delporte (1957).

Table 5 - Tool-types from the Mousterian levels of the Grotte des Fées. After Bailleau (1872) and Delporte (1957).

(20) "Finally, we would ask why none of the upper, supposedly backdirt levels (B1-B3) yielded any trace of typically Mousterian artefacts (...) because we know that the basal Mousterian levels were extensively excavated during the 19th century work on the site". 


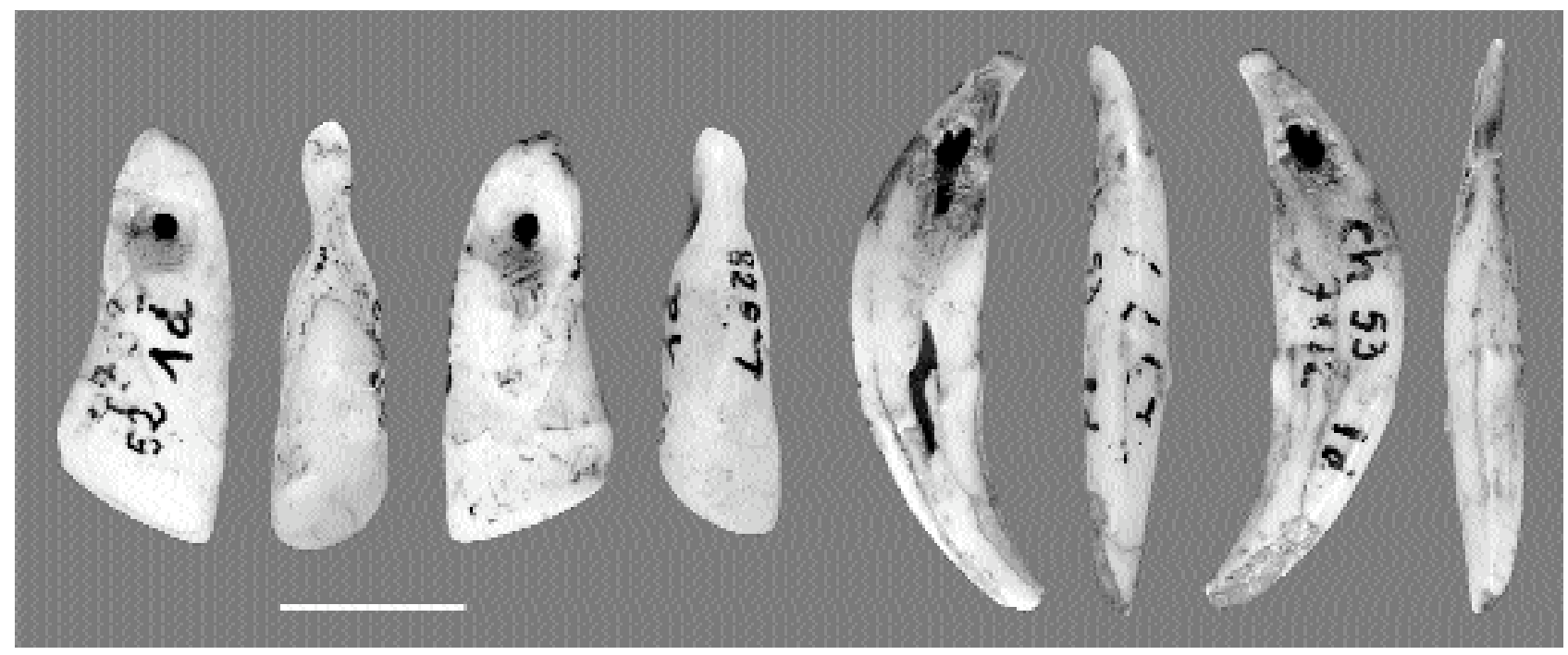

Figure 24 - Objets de parure recueillis par Delporte dans le niveau 1d (= B4). A droite : canine de Renard provenant de la campagne de fouille 1953 montrant une perforation obtenue par raclage longitudinal. A gauche : canine de Cerf très usée provenant de la campagne de fouille 1952 et montrant une perforation obtenue par rotation. Trait : $1 \mathrm{~cm}$.

Figure 24 - The ornaments found by Delporte in level 1d (=B4). Right : fox canine from the 1953 field season with perforation obtained by longitudinal scraping heavily worn. Left : red deer canine from the 1952 field season with perforation obtained mostly by rotation. Scale bar $=1 \mathrm{~cm} t$

Bien que cela puisse surprendre Mellars et alii, le nombre de " pièces typiquement moustériennes " est très faible au sein des collections du XIX e siècle (tabl. 5). L'inventaire de Delporte (1957) ne fait apparaître que six pièces et sa propre collection (fig. 23), avec onze objets, est à peine plus riche. Le contraste est important avec les 203 pièces retouchées que Delporte a décompté dans la série lithique provenant des niveaux $B$ (fig. 10). Cette différence significative entre les niveaux moustériens et châtelperroniens a été soulignée sans ambiguité par Delporte : "Malgré la faible surface de l'espace " fouillable ", une différence fondamentale a pu être constatée entre les niveaux nets de la couche supérieure et la diffusion de vestiges, ainsi que leur petit nombre, dans la couche inférieure ; cette pauvreté de l'industrie est d'ailleurs confirmée par l'étude de la série Bailleau, beaucoup plus riche en pièces du Paléolithique supérieur qu'en pièces moustériennes" (Delporte 1957 : 457-458).

En somme, il n'y a aucune raison de croire que Bailleau ait intensément fouillé les niveaux moustériens. Peut-être lui sont-ils surtout apparus comme des niveaux d'argiles stériles. Mais, même si cela a été le cas, ses chances de recueillir des vestiges (et donc qu'une fraction ait alimenté les déblais) restent extrêmement faibles. L'absence de pièce moustérienne n'est donc pas une surprise, pas plus dans le niveau $A$ que dans les niveaux $B 1$ à $B 3$.

\section{6 - OBJETS DE PARURE}

Mellars et ses collaborateurs considèrent que les deux dents percées provenant du niveau B4 sont aurignaciennes. À aucun moment, ils n'envisagent qu'elles puissent être châtelperroniennes :

"Bien que Zilhão et al. (...) suggèrent que ces dernières [les deux dents perforées (...) également recueillies par Delporte dans ce niveau] pourraient être aussi bien des objets châtelperroniens (par comparaison avec les découvertes de la grotte du Renne à Arcy-sur-Cure), une des dents en question a déjà été décrite par Randall White (...) comme présentant une technique de perforation " compatible avec les centaines de dents percées aurignaciennes que j'ai examinées mais dont la technique de perforation se distingue de celle que j'ai observée (...) à Arcy ou à Quinçay ". Nous remarquons qu'aucun autre spécimen de dent perforée n'a été recueilli dans tous les autres niveaux châtelperroniens, que ce soit le niveau de base (B5) ou les niveaux supérieurs (B1-B3) " ${ }^{21}$ (p. 3660).

Nous notons que Mellars et alii acceptent notre diagnostic à savoir que la dent qu'ils ont dans un premier temps décrite comme appartenant à un grand félin (Gravina et al. 2005 : fig. 3) est en fait une canine de Cerf

(21) "Although Zilhão et al. (...) suggest that the latter [the two perforated animal tooth pendants (...) also recovered by Delporte from this level] could conceivably be Chatelperronian specimens (by analogy with the finds from the Grotte du Renne at Arcy-surCure), one of the teeth in question has already been described by Randall White (...) as showing techniques of perforation 'consistent with that of the hundreds of Aurignacian pierced teeth I have examined, and in contrast with the hole perforation techniques I have observed (...) at Arcy and Quinçay'. We note that not a single additional specimen of a perforated tooth was recovered from any of the other levels at Châtelperron, from either the basal (B5) or overlying (B1-B3) Chatelperronian levels". 
très usée (Zilhão et al. 2006: 12648 et fig. 24). Sur la base des critères diagnostiques publiés, provenant de l'étude de deux populations de cerfs d'Angleterre et d'Ecosse et dont l'âge et le sexe étaient connus (d'Errico et Vanhaeren 2002), nous pouvons maintenant ajouter que cette dent était probablement celle d'une biche âgée de 14 à 16 ans.

Auparavant, Mellars et alii ont également décrit les deux parures comme réalisées par "un fin raclage amincissant la racine, suivi de la perforation du trou " ${ }_{22}$ (Gravina et al. 2005 : 53), alors qu'ils semblent maintenant penser que cette technique n'a été employée que pour l'une des deux dents, mais ils ne précisent pas laquelle. Notre analyse montre qu'en fait, les deux dents ont été confectionnées selon des techniques différentes. La perforation de la canine de Renard est faite entièrement par un raclage longitudinal de la racine à l'aide d'une pointe de silex tandis que la canine de biche a été perforée par rotation avec des traces de raclage précédant et suivant la perforation.

Ces différences ne peuvent être interprétées que de deux façons : soit les deux techniques ont coexisté (dans l'Aurignacien, dans le Châtelperronien, ou les deux), ce qui interdirait la possibilité d'une attribution culturelle de parures issues de séries hétérogènes sur la base de leur technique de perforation ; soit les deux parures relèvent de deux cultures distinctes, peut-être d'époques différentes, ce qui s'oppose à l'affirmation de Mellars et alii selon laquelle ces deux parures sont aurignaciennes. Pour trancher entre ces deux possibilités, il serait nécessaire de disposer d'une meilleure connaissance de la variabilité des techniques de confection des parures à l'Aurignacien et au Châtelperronien. Une base de données exhaustive existe pour l'Aurignacien (Vanhaeren et d'Errico 2006), mais le fait que la majeure partie des parures châtelperroniennes fiables soient issues de deux sites partiellement publiés, Quinçay (Granger et Lévêque 1997) et la grotte du Renne à Arcysur-Cure (White 2001), ne permet pas de conclusions définitives.

Dans l'état actuel des connaissances, on ne peut se baser que sur le fait que des parures en canine de Cerf ou de Renard sont connues aussi bien à l'Aurignacien qu'au Châtelperronien, ce qui aurait dû modérer la confiance montrée par Mellars et alii dans une attribution des deux spécimens de la grotte des Fées à l'Aurignacien. La technique de raclage employée pour la canine de Renard est connue au Châtelperronien. Elle est, en particulier, décrite par White (2001 : tableau 5) pour deux phalanges percées de Renne des niveaux IX et $X$ de la grotte du Renne. Mais la technique de perçage par rotation utilisée pour la canine de biche est, à ce jour, inconnue avant l'Aurignacien, bien que quelques canines de Cerf de Quinçay présentent de larges perforations subcirculaires très usées qui auraient pu être obtenues par rotation (Granger et Lévêque 1997 : fig. A; Soressi et d'Errico 2007). Si la canine de biche était de facture aurignacienne, cela ne témoignerait pas pour autant d'une interstratification puisque, si elle est issue du niveau B4, elle a été mise au jour en 1952, c'est-à-dire l'année où Delporte a traversé les déblais dans la tranchée longitudinale (cf. supra et fig. 3,9).

\section{7 - COMPÉTENCES D'HENRI DELPORTE}

Mellars et ses collaborateurs insistent fortement sur les compétences de fouilleur d'Henri Delporte :

" Peut-être la chose la plus extraordinaire (...), dans l'hypothèse de déblais énoncée par Zilhão et al., est le remarquable degré d'incompétence qu'elle implique quant à la conduite des fouilles et la compréhension qu'en a eu Delporte à Châtelperron " ${ }^{23}$ (p. 3661).

Nous sommes en désaccord avec l'opinion selon laquelle discuter l'interprétation stratigraphique d'Henri Delporte revient à le considérer comme incompétent. Dans une discipline où le recueil même des données engendre leur destruction, la condition de reproductibilité de l'observation est compromise. II s'agit d'un point méthodologique qui place déjà la connaissance archéologique en porte-à-faux vis-à-vis de la méthode scientifique, en particulier lorsque l'absence de témoin ne permet pas de nouvelles fouilles. Aussi, la discussion des informations objectives livrées par la fouille (p. ex. photographie, ensembles archéologiques) est plus que jamais nécessaire pour inscrire l'archéologie dans le champ de la connaissance scientifique. L'argument d'autorité aux accents médiévaux que présentent Mellars et alli nous paraît extrêmement dangereux car il constitue une grave entorse à la méthode scientifique, qui admet l'erreur au sein de sa démarche et où le progrès est souvent le fruit d'une correction des erreurs passées (fussent-elles les nôtres).

Nous avons soutenu précédemment (Rigaud 2000, 2001 ; Bordes J.-G. 2002, 2003, 2006) que d'autres personnes faisant autorité dans la discipline ont fait de semblables erreurs. Un exemple notable est donné par la mise en cause de l'interprétation de la stratigraphie du Roc-deCombe par F. Bordes, l'un des archéologues les plus influents du $X X^{e}$ siècle, et que Mellars et ses collaborateurs ont admis (p. 3662). Nous nous opposons avec force à l'idée que, ce faisant, Mellars et ses collaborateurs accusent implicitement F. Bordes de grave incompétence dans la conduite des fouilles et la compréhension du site de Roc-de-Combe.

(22) "initial scraping to thin the root of the tooth, followed by perforation of the hole".

(23) "Perhaps the most extraordinary (...) aspect of the Zilhão et al. backdirt hypothesis is the remarkable degree of archaeological incompetence it implies in the conduct and recording of Delporte's excavations at Châtelperron" 


\section{8 - DISCUSSION}

La grotte des Fées a été intensément fouillée avant que Delporte n'en reprenne l'étude dans les années cinquante. Ces demières fouilles n'ont jamais donné lieu à un enregistrement précis ni à une publication exhaustive sur laquelle puisse s'appuyer une évaluation détaillée des différentes interprétations. Les objets n'ont pas été coordonnés et, pour la plupart des campagnes de fouille, il n'y a pas d'indication du secteur d'où proviennent les pièces. Comme nous l'avons souligné (Zilhão et al. 2006, p. 12647), cet état de fait limite la connaissance que l'on peut avoir de la configuration initiale du site et de sa stratigraphie. La principale conséquence est que toute interprétation de ce site laissera des zones d'ombre.

Mellars et ses collaborateurs (2007 : 3657) ont fait beaucoup de cas de ce qu'ils ont perçu comme une évolution dans les " raisons précises pour laquelle l'interprétation de la séquence de Châtelperron était rejetée ", entre notre publication (Zilhão et al. 2006) et une première présentation de notre analyse à la réunion annuelle de la Paleoanthropology Society). Mellars et al. loc. cit. semblent avoir vu une contradiction entre notre proposition initiale qu'" au moins, les niveaux B1-B3 et, en toute vraisemblance, toute la séquence $\mathrm{B} 1-\mathrm{B} 5$, représentent des dépôts remaniés, et le matériel archéologique des déblais issus des fouilles pratiquées au $\mathrm{XIX}^{\mathrm{e}}$ siècle sur le site " ${ }^{24}$, et la version finalement publiée de notre hypothèse, à savoir que " le matériel des niveaux châtelperroniens les plus bas (les niveaux B4 et B5 de Delporte) étaient, en fait, presque certainement en place et (...) seul le matériel de niveaux châtelperroniens sus-jacents (les niveaux B1-B3) doit (...) être interprété comme une accumulation de déblais du XIX ${ }^{\text {e }}$ siècle ${ }^{25}$. Alors que, pour la première version, les auteurs citent intégralement le résumé du colloque, la soi-disant version finale ne semble pas venir d'ailleurs que de l'imagination de Mellars et alii. Le texte original est le suivant :

"Ces données [l'évolution verticale de la fréquence des caractères de l'industrie lithique pertinents du point de vue taphonomique] suggèrent que, si les niveaux B5 et B4a peuvent bien avoir été plus ou moins en place, les niveaux $B 1$ et B2 ne le sont pas. Etant donnée l'augmentation importante de bords endommagés entre B4 à B3, cette même interprétation s'applique probablement à ce dernier niveau " ${ }^{26}$ (p. 12647).
Nous avons également écrit que, des deux seules interprétations possibles de la séquence, à savoir : soit l'ensemble des niveaux $B$ est " entièrement perturbé " ${ }^{27}$, soit les niveaux B1-B3a sont remaniés et les niveaux B4-B5a " des occupations châtelperroniennes en place contenant quelques intrusions aurignaciennes ", la demière a été préférée du fait de " notre analyse et de la lecture stratigraphique faite par Delporte en 1962, qui avait alors regroupé les différents niveaux en trois ensembles " (ibidem). Nous n'avons pas écrit que cette seconde hypothèse était " presque certaine ", et nous avons encore moins écrit que nos analyses excluaient la première hypothèse.

$\mathrm{Si}$, d'une part, le site avait originellement présenté, au-dessus de couches moustériennes presque stériles les vestiges d'une occupation châtelperronienne très riche, recouverts d'une très fine lentille de matériel aurignacien et si, d'autre part, les fouilles Bailleau avaient entièrement bouleversé ces niveaux jusqu'au sommet des dépôts moustériens, quel aurait été le contenu des déblais Bailleau ? II aurait essentiellement consisté en pièces châtelpenoniennes éparpillées, en ossements, avec quelques objets aurignaciens dispersés, soit exactement ce que nous pouvons observer de la série lithique des niveaux $B$ de Delporte. Dans ce cas, et à moins qu'un nombre suffisamment important d'échantillons soit daté, des datations ${ }^{14} \mathrm{C}$ peuvent toujours offrir des résultats cohérents. Par exemple, si l'on suppose que la distribution des ossements suit la proportion de vestiges lithiques aurignaciens ou châtelperroniens, dont le rapport est de 1/16 (Zilhão et al. 2006 : tableau 4), obtenir trois dates compatibles avec le Châtelperronien, à partir de trois échantillons du niveau B5, n'est pas, en soi, suffisant pour montrer que ce niveau est homogène ou, simplement, qu'il n'est pas remanié.

Notre reconnaissance de la présence de matériel recueilli dans des témoins intacts, au sein des séries correspondant aux niveaux B4 et B5, se base sur les données topographiques, et non pas sur les datations ${ }^{14} \mathrm{C}$. Le fort contraste entre les niveaux B et les couches moustériennes sous-jacentes - une " argile jaune beaucoup plus compacte et homogène " (Delporte $1955: 83$ ) - suggère qu'un dégagement fidèle de la base de la fouille Bailleau dans les niveaux moustériens n'était pas une tâche difficile et, donc, que le report que fait Delporte sur son propre plan, des limites de la fouille Bailleau est digne de confiance. Cela étant, et en dépit de l'évasement noté par Delporte, un espace suffisant existe pour que, entre l'ancienne fouille et les parois de la cavité, des témoins châ-

(24) "at least Châtelperron levels B1-B3, and in all likelihood the entire B1-B5 sequence, represent reworked sediments and archaeological material, probably backdirt from the excavations conducted on the site in the nineteenth century".

(25) "the material from the basal Chatelperronian levels (Delporte's levels B4 and B5) was in fact almost certainly in situ and (...) only the material from the overlying, upper Chatelperronian levels (levels B1-B3) should (...) be interpreted as a 19th century 'backdirt' accumulation."

(26) "These data [the vertical variation in the frequencies of taphonomically significant lithic attributes] suggest that, whereas levels B4 to B5a may well have been broadly in situ, B1 and B2 were not; given the marked surge in edge-damaged items from B4 to B3, the same probably applies to the latter".

(27) "entirely disturbed". 
telperroniens aient été préservés, à la base de la séquence, et fouillés (cf. fig. 1,3,9,13). En même temps, dans la mesure où certaines des pièces des niveaux B4 et B5 (en fait " 1d" et " 1e ") portent la mention de l'année 1952 et, qu'en 1952, les travaux de Delporte ont consisté en la réalisation d'une tranchée traversant le déblai Bailleau dans la partie centrale du site, nous sommes assurés que les niveaux B4 et B5 contiennent également du matériel issu de dépôts remaniés. Cela n'invalide pas notre précédente comparaison entre les deux regroupements de niveaux (Zilhão et al. 2006 : fig. 3) : si B1 à B3 sont exclusivement des déblais, une proportion importante du matériel in situ des niveaux B4-B5 gardera une cohérence, comme le traduit la distribution des fréquences des différents critères taphonomiques que nous avons utilisés (cf. fig. 14).

Le choix d'une mise en perspective différente (et non pas d'un changement de point de vue) entre la communication présentée à la Paleoanthropology Society et l'article paru dans les comptes-rendus de l'Académie américaine des Sciences est lié au fait que, dans cette demière publication, nous nous sommes d'abord attaché aux implications paléoanthropologiques de l'interstratification plutôt qu'à expliquer la grotte des Fées en elle-même. Considérant le sujet traité, il était suffisant de montrer que les niveaux B1 à B3 étaient remaniés. Pour cette raison, nous n'avons pas jugé nécessaire de clarifier précisément le statut des niveaux B4 à B5 par un exposé détaillé. Aussi, nous avons admis qu'en dépit du fait qu'ils contenaient du matériel aurignacien, les niveaux B4 et B5 étaient " grossièrement en place ". Toutefois, et les éléments que nous avons exposés précédemment montrent que cela est loin d'être certain, une forte présomption de non remaniement existe pour le matériel recueilli dans les niveaux B4 et B5 en 1962 , sans que cela n'engage le statut du matériel récolté au cours des campagnes de fouilles 1952-1954 dans ces mêmes niveaux.

L'exposé de nos vues sur la stratigraphie de la grotte des Fées qui, nous l'espérons, ne prêtera pas à de nouvelles querelles de grammairiens, peut finalement être résumé comme suit :

- nous n'avons aucun doute quant au fait que les niveaux B1 à B3 des fouilles Delporte de la grotte effondrée sont des déblais et nous croyons que le matériel des niveaux B4-B5 provient, pour partie et bien que nous n'en connaissions pas la proportion, mais pour partie seulement, de témoins intacts du remplissage originel de la cavité ;

- le site contenait à l'origine un riche ensemble châtelperronien couvert par des niveaux superficiels dans lesquels étaient inclus des vestiges représentant des incursions sporadiques, accumulés au cours d'occupations aurignaciennes plus tardives.

- il est possible que les échantillons du niveau B5 datent, en réalité, des témoins in situ à la périphérie de l'habitat châtelperronien fouillé par Bailleau au centre de la cavité, et que les échantillons des niveaux B1-B3 datent des vestiges accumulés dans une tanière de camivores postérieure aux occupations châtelperroniennes et située dans un renfoncement de la paroi sud de la grotte, hors de la zone d'occupation humaine.

\section{9 - CONCLUSION}

L'exposé qui précède fait clairement apparaître que ce qui nous distingue de Mellars et alii est d'abord une différence de démarche. Pour nos collègues, il s'est agit de prélever des échantillons dans les collections du MAN, de les envoyer au laboratoire de datation et d'interpréter les résultats obtenus en se basant sur la stratigraphie du site publiée un demi-siècle auparavant. À aucun moment, ils n'ont examiné les vestiges, ou étudié la collection d'ossements d'où ont été extraits leurs échantillons, dans le but d'évaluer les agents de formation du site, le degré d'intégrité de la stratigraphie ou la pertinence de ces échantillons vis-à-vis des questions archéologiques abordées. En somme, leur approche fait tout simplement l'impasse sur toute forme de "Quellenkritik". Nous pensons que la recherche en archéologie ne peut plus être menée de cette façon, au $X X I^{e}$ siècle, et nous espérons que, indépendamment de la question posée de l'interstratification à la gro tte des Fées, cette controverse aura aidé à la mise au point de protocoles à suivre dans l'étude de collections anciennes. Cela n'en est que plus nécessaire encore dans le cas de datations d'objets issus de telles collections. En effet, dans la mesure où la précision et l'efficacité des techniques de datation ont été considérablement améliorées, la question de l'association et de la signification des objets échantillonnés qui est et ne demeure qu'un problème archéologique, n'en est que plus cruciale.

L'absence d'examen critique des sources par Mellars et alii les conduit, en fin de compte, à une position intenable sur deux points. D'abord, dans la mesure où ils ne peuvent que se baser sur nos propres analyses, ils sont portés à un double biais : accepter la fiabilité des ces analyses si les résultats de ces demières leur conviennent, ou la rejeter en se contentant d'un " sentiment en leur for intérieur" (Dawkins 2006) si le résultat ne s'accorde pas à leur conception du site. Ensuite, ils n'ont d'autre solution que d'avancer pour ultime argument que la compétence de fouilleur d'Henri Delporte suffit à valider la stratigraphie de la grotte des Fées. Ce second point soulève la question de savoir ce qu'apporte la datation d'un site s'il est admis que le fouilleur n'a pu se tromper, quand bien même les datations obtenues indiqueraient le contraire?

Lorsque les questions des peuplements néanderthaliens et des hommes anatomiquement modernes et de l'interstratification sont abordées, Mellars et alii commettent l'erreur de croire que la prétendue réfutation de notre interprétation suffit à confirmer la leur. Mais, indépendamment des problèmes que peut soulever l'interprétation de la grotte des Fées de Zilhão et al. (2006), Mellars et alii doivent encore démontrer la justesse de leur vue. Un point élémentaire de logique qu'ils ont négligé est, qu'en obtenant des âges châtelperroniens à partir d'échantillons issus des 
déblais d'un site châtelperronien, ils ont montré, dans le meilleur des cas, que les déblais viennent bien du site, et non pas qu'il s'agit d'un témoin intact (un exemple analogue est donné par le travail récent mené sur le site éponyme de Néandertal - Schmitz et al. 2002). De plus, en construisant leur raisonnement sur la réfutation de nos conclusions, Mellars et alii ont également laissé échapper un second point de logique, celui de savoir à qui incombe la charge d'apporter la preuve. Des quatre exemples d'interstratification qui ont été proposés à ce jour, ils ne remettent pas en cause le fait que trois doivent être rejetés (Roc-de-Combe, Le Piage et El Pendo) et que, au cours de ces cinquante dernières années, aucun autre site fouillé avec des techniques modemes n'a livré quelque exemple qui s'en approche. La défense du modèle de l'interstratification, avec ses implications sur le débat de la transition, nécessiterait donc de disposer d'un exemple qui ne laisse aucune place au doute. Comme nous l'avons montré, ce n'est pas le cas de la grotte des Fées et notre interprétation critique rend bien mieux compte de l'intérêt du site et des séries recueillies.

Cela ne veut pas dire que l'interstratification Aurignacien / Châtelperronien n'existe nulle part. Dans leurs conclusions, Mellars et alii (2007 : 3662) considèrent que la localisation de la Grotte des Fées est importante. Pour eux, l'interstratification pourrait ne pas être observable dans les sites du Périgord, mais le serait dans l'Allier car cette région est géographiquement plus proche des régions d'où viennent les populations d'hommes anatomiquement modernes. Cette région serait par conséquent, plus susceptible de livrer des sites témoignant du flux et du reflux le long de la zone de contact entre les régions à peuplement néandertalien et modeme. Si tel était le cas, cette prédiction devrait être vérifiée par la recherche et la découverte de nouveaux sites situés à proximité de la grotte des Fées. Tout comme dans les autres disciplines scientifiques, la preuve ultime est la mise à l'épreuve des hypothèses par la vérification des prédictions. Nous sommes convaincus qu'une telle situation de recouvrement territorial implique un temps trop court pour permettre un enregistrement stratigraphique en grotte ou sous abri, et c'est la raison pour laquelle aucun exemple incontestable de contemporanéité entre les populations châtelpenoniennes et aurignaciennes n'a été découvert à ce jour. Prouver le contraire est le défi de Mellars et de ses collaborateurs, pas le nôtre.

\section{Remerciements}

Nous remercions l'équipe du Musée d'Archéologie nationale de Saint-Germain-en-Laye et particulièrement Catherine Schwab, Marie-Sylvie Larguèze et Sandra Bercut pour leur précieuse assistance, Marian Vanhaeren et Boris Valentin pour leur aide dans la recherche de matériel iconographique et de certaines références bibliographiques, Jacques Pelegrin pour des précisions concemant la possible pièce solutréenne du niveau B2, Paul Bahn, Alistair Pike, Erik Trinkhaus et Donald Grayson pour leurs commentaires à un première version du manuscrit. Ce travail a été financé par les programmes «origine de l'Homme, des langues et du langage » (European Science
Foundation), «Environnements et Climats du passé » (CNRS), «Transmissions - D'une société à l'autre : processus d'adaptation et de peuplement » (Région Aquitaine) et le fonds pour la recherche de la faculté des arts de Bristol.

\section{BIBLIOGRAPHIE}

AUBRY Th. 1991 - L'exploitation des ressources en matières premières lithiques dans les gisements solutréens et badegouliens du bassin versant de la Creuse (France). Thèse de doctorat, Université de Bordeaux 1, $327 \mathrm{p}$.

BAILLEAU J.-G. 1869 - Grotte des Fées de Châtelperron. Moulins : Desrosiers, 21 p., 3 pl.

BAILLEAU J.-G. 1872 - L'homme pendant la période quatemaire dans le Bourbonnais. In : Congrès Scientifique de France, Trente-septième session (août 1870). Tome II. Moulins : Desrosiers, p. 95-130.

BERNALDO DE QUIRÓS F. 1982 - Los inicios del Paleolítico Superior Cantabrico. Madrid : Centro de Investigación y Museo de Altamira, 8, 347 p.

BINFORD L. 1973 - Interassemblage variability: The Mousterian and the "functional" argument. In : C. RENFREW (Ed.), The Explanation of Culture Change. Models in Prehistory London : Duckworth, p. 227-254.

BON F. 2002 - L'Aurignacien entre Mer et Océan. Réflexion sur l'unité des phases anciennes de l'Aurignacien dans le sud de la France. Paris : Mémoire de la Société Préhistorique Française XXIX, 253 p.

BORDES F. 1968 - La question périgordienne. In : La Préhistoire problèmes et tendances. Paris : Centre National de la Recherche Scientifique, p. 59-70.

BORDES F. 1973 - On the chronology and contemporaneity of different paleolithic cultures in France. In : C. Renfrew (Ed.), The Explanation of Culture Change. Models in Prehistory. London : Duckworth, p. 217-226.

BORDES F., LABROT J. 1967 - Stratigraphie de la grotte de Roc-de-Combe (Lot) et ses implications. Bulletin de la Société Préhistorique Française, 64, p. 15-28.

BORDES J.-G. 2002 - Les interstratifications Châtelperronien/Aurignacien du Roc de Combe et du Piage (Lot, France). Analyse taphonomique des industries lithiques: conséquences archéologiques. Thèse de doctorat, Université Bordeaux1, $365 \mathrm{p}$.

BORDES J.-G. 2003 - Lithic taphonomy of the Châtelperronian/Aurignacian interstratifications in Roc de Combe and Le Piage (Lot, France). In : J. Zilhão, F. d'Errico (Eds.), The Chronology of the Aurignacian and of the Transitional Technocomplexes. Dating, Stratigraphies, Cultural Implications. Lisbonne: Trabalhos de Arqueologia 33, Instituto Português de Arqueologia, p. 223-244. 
BORDES J.-G. 2006 - News from the West: a reevaluation of the classical Aurignacian sequence of the Périgord. In : O. Bar-Yosef, J. Zilhão (Eds.), Towards a Definition of the Aurignacian. Lisbonne : Trabalhos de Arqueologia 45, American School of Prehistoric Research/Instituto Português de Arqueologia, p. 147-171.

CHAMPAGNE F., ESPITALIÉ R. 1981 - Le Piage, site préhistorique du Lot. Paris, Mémoire de la Société Préhistorique Française, 15, 207 p.

D'ERRICO F. 2003 - The Invisible Frontier. A Multiple Species Model for the Origin of Behavioral Modemity. Evolutionary Anthropology, 12, p. 188-202.

D'ERRICO F., VANHAEREN M. 2002 - Criteria for Identifying Red Deer (Cervus elaphus) Age and Sex from Their Canines. Application to the Study of Upper Palaeolithic and Mesolithic Ornaments. Joumal of Archaeological Science, 29, p. 211-232.

D'ERRICO F., ZILHÃO J., BAFFIER D., JULIEN M., PELEGRIN J. 1998 - Neanderthal Acculturation in Westem Europe? A Critical Review of the Evidence and Its Interpretation Current Anthropology, 39, supplément, p. S1-S44.

DAWKINS R. 2006 - The God Delusion, London, Bantam, 406 p.

DELPORTE H. 1952 - Note préliminaire sur la stratigraphie et d'industrie de Châtelperron (Allier), Montbrison

DELPORTE H. 1955 - Les fouilles des grottes paléolithiques de Châtelperron (Allier). Gallia, 13, p. 79-84.

DELPORTE H. 1957 - La Grotte des Fées de Châtelperron (Allier). In : Congrès Préhistorique de France. Compterendu de la XVème Session. Paris : Société Préhistorique Française, p. 452-477.

DELPORTE H. (n.d.) - La station de Châtelperron. Manuscrit inédit (ca. 1964, conservé au Musée d'Archéologie Nationales, Saint-Germain-en-Laye).

DELPORTE H., SURMELY F., URGAL A 1999 Châtelperron: Un grand gisement préhistorique de l'Allier. Moulins, Conseil General de l'Allier, 48 p.

DEMARS P.-Y., HUBLIN J.-J. 1989 - La transition néandertaliens/hommes de type moderne en Europe occidentale : aspects paléontologiques et culturels. In : M. Otte, $\mathrm{H}$. Laville (Eds.), L'Homme de Néandertal 7: l'extinction. Liège : Études et Recherches Archéologiques de I'Université de Liège 34, p. 29-42.

DEMARS P.-Y., LAURENT P. 1989 - Types d'outils lithiques du Paléolithique supérieur en Europe, Paris : Centre National de la Recherche Scientifique, $178 \mathrm{p}$.

DEPRAETERE B. 2000 - Technologie lithique châtelperronienne. Présentation et étude du matériel lithique de
Châtelpenon (Allier). Mémoire de maîtrise, Université Paris1, $90 \mathrm{p}$.

DUJARDIN V. 2001 - Sondages à la Quina aval (Gardesle-Pontaroux, Charente, France). Antiquités Nationales, 33, p. 21-26.

GRAVINA B., MELLARS P., BRONK RAMSEY C. 2005 Radiocarbon dating of interstratified Neanderthal and early modem human occupations at the Chatelperronian typesite. Nature, 438, p. 51-56.

GRANGER J.-M., LÉVÊQUE F. 1997 - Paru re castelperronienne et aurignacienne: étude de trois séries inédites de dents percées et comparaisons. Comptes-Rendus de l'Académie des Sciences de Paris, 325, p. 537-543.

HARROLD F. B. 1988 - The Chatelperronian and the Early Aurignacian in France. In : J. F. HoffeckerR, C. A. Wolf (Eds.), The early Upper Paleolithic: evidence from Europe and the Near East. Oxford, British Archaeological Reports Intemational Series 437, p. 157-191.

HENRY-GAMBIER D., MAUREILLE B., WHITE R. 2004 Vestiges humains des niveaux de l'Aurignacien ancien du site de Brassempouy (Landes). Bulletins et Mémoires de la Société d'Anthropologie de Paris, 16, (1-2), p. 49-87.

HIGHAM T. F. G., JACOBI R. M., BRONK RAMSEY C. 2006 - AMS radiocarbon dating of ancient bone using ultrafiltration. Radiocarbon, 48 (2), p. 179-195.

HUBLIN J.-J. 2000 - Modern-non modern hominid interactions: A Mediterranean perspective. In : O. Bar-Yosef, D. Pilbeam (Eds.), The Geography of Neandertals and Modern Humans in Europe and the Greater Mediteranean. Cambridge : Peabody Museum Bulletin; 8, p. 157-182.

HUBLIN J.-J., SPOOR F., BRAUN M., ZONNEVELD F., CONDEMI S. 1996 - A late Neanderthal associated with Upper Palaeolithic artefacts. Nature, 381, p. 224-226.

INIZAN M-L., ROCHE H., TIXIER J. 1992 - Technology of knappped stone. Valbonne : Centre de Recherches et d'Études Préhistoriques, $127 \mathrm{p}$.

JÖRIS O., ÁlVAREZ E., WENINGER B. 2003 - Radiocarbon Evidence of the Middle to Upper Paleolithic Transition in Southwestern Europe. Trabajos de Prehistoria, 60 (2), p. 15-38.

KLARIC L. 2003 - L'unité technique des industries à burins du Raysse dans leur contexte diachronique. Réflexions sur la diversité culturelle au Gravettien à partir des données de la Picardie, d'Arcy-sur-Cure, de Brassempouy et du Cirque de la Patrie. Thèse de doctorat, Université Paris 1, 427p.

LACAILLE A. D. 1947 - Châtelperron: a new survey of its Palaeolithic industry. Archaeologia, 92, p. 95-119.

LAVILLE H., RIGAUD J.-Ph., SACKETT J. 1980 - Rock shelters of the Périgord. New York : Academic Press, 371 p. 
LÉVÊQUE F., VANDERMEERSCH B. 1980 - Découverte de restes humains dans un niveau castelperronien à SaintCésaire (Charente-Maritime). Comptes rendus de l'Académie des Sciences de Paris, 291, série D, p. 187-189.

PELEGRIN J. 1995 - Technologie lithique: le Châtelpemonien de Roc-de Combe (Lot) et de la Côte (Dordogne). Paris : éditions du Centre national de la recherche Scientifique, 297 p.

MELLARS P. A. 1999 - In: The Neanderthal Problem Continued.Current Anthropology, 40 (3), p. 341-350.

MELLARS P. A., GRAVINA B; BRONK RAMSEY C. 2007 Confirmation of Neanderthal/modern human interstratification at the Chatelperronian type-site. Proceedings of the National Academy of Sciences USA, 104 (9), p. 3657-3662.

MELLARS P. A., STRINGER C. B. (eds.) 1989 - The Human Revolution. Edinburgh : Edinburgh University Press, $810 \mathrm{p}$.

MONTES R., SANGUINO J. (Eds.) 2001 - La Cueva del Pendo. Actuaciones Arqueológicas 1994-2000. Santander: Ayuntamiento de Camargo / Gobierno de Cantabria / Parlamento de Cantabria, $279 \mathrm{p}$.

MONTES R., SANGUINO J., MARTÍN P., GÓMEZ A. J., MORCILLO C. 2005 - La secuencia estratigráfica de la cueva de El Pendo (Escobedo de Camargo, Cantabria): problemas geoarqueológicos de un referente cronocultural. In : M. Santonja, A. Pérez-Gonzalez, M. Machado (Eds.), Geoarqueología y patrimonio en la Península Ibérica y el entorno mediterráneo. Almazán (Soria) : ADEMA, p. 127-138.

PEYRONY D. 1933 - Les industries «aurignaciennes « dans le bassin de la Vézère. Bulletin de la Société Préhistorique Française, 30, p. 543-559.

PEYRONY D. 1948 - Le Périgordien, l'Aurignacien et le Solutréen d'après les demières fouilles. Bulletin de la Société Préhistorique Française, 45, p. 305-328.

RIGAUD J.-Ph. 2000 - Late neandertals in the southwest of France and the emergence of the Upper Paleolithic. In : C. Stringer, R. N. E. Barton, J. C. Finlayson (Eds.), Neanderthals on the edge: papers from a conference marking the 150th anniversary of the Forbes' Quarry discovery, Gibraltar. Oxford : Oxbow, p. 27-31.

RIGAUD J.-Ph. 2001 - À propos de la contemporanéité du Châtelperronien et de l'Aurignacien ancien dans le nordest de l'Aquitaine: une révision des données et ses implications. In : J. Zilhão, Th. Aubry, A. F. Carvalho (Eds.), Les premiers hommes modernes de la Péninsule Ibérique. Lisbonne : Trabalhos de Arqueologia 17, Instituto Português de Arqueologia, p. 61-68.

SCHMITZ R. W., SERRE D., BONANI G., FEINE S., HILLGRUBER F., KRAINITZKI H., PÄÄBO S., SMITH F. H. 2002 - The Neandertal type site revisited: Interdisciplinaryinves- tigations of skeletal remains from the Neander Valley, Gemany. Proceedings of the National Academy of Sciences USA, 99, p. 13342-13347.

SMITH Ph. 1966 - Le Solutréen en France. Bordeaux : Delmas, $449 \mathrm{p}$.

SONNEVILLE-BORDES D. de 1982 - L'évolution des industries aurignaciennes. In : Aurignacien et Gravettien en Europe, II, Cracovie - Nitra - 1980. Liège : Études et Recherches Archéologiques de l'Université de Liège 13, p. 339-360.

SORESSI M., D'ERRICO F. 2007 - Pigments, gravures, parures : les comportements symboliques controversés des Néandertaliens. In : B. Vandermeersch, B. Maureille (Eds.), Les Néandertaliens. Biologie et cultures. Paris : Documents Préhistoriques 23, Éditions du CTHS, p. 297-309.

STRINGER C., GAMBLE C. 1993 - In Search of the Neanderthals, London, Thames and Hudson, 247 p.

TEXIER J.-P., KERVAZO B., LENOBLE A., NESPOULET R. 2004 - Sédimentogenèse des sites préhistoriques du Périgord. Association des Sédimentologues Français, 83 p.

TIXIER J. , INIZAN M.-L. et ROCHE H. 1980 - Préhistoire de la Pierre Taillée. 1-Terminologie et technologie. CREP Antibes.

TRINKAUS E., MOLDOVAN O., MILOTA ., BÎLG R A., SARCINA L., ATHREYA S., BAILEY S. E., RODRIGO R., MIRCEA G., HIGHAM Th., BRONK RAMSEY C., PLICHT J. 2003 - An early modern human from the Pe tera cu Oase, Romania. Proceedings of the National Academy of Sciences USA, 100, p. 11231-11236.

VANHAEREN M., D'ERRICO F. 2006 - Aurignacian ethnolinguistic geography of Europe revealed by personal ornaments. Journal of Archaeological Science, 33, p. 1105-1128.

VILLA P., D'ERRICO F. 2001 - Bone and ivory points of the Lower and Middle Paleolithic of Europe. Journal of Human Evolution, 41, p. 69-112.

WENINGER B., JÖRIS O. 2007 - The Cologne Radiocarbon Calibration \& Paleoclimate Research Package (http://www.calpal.de).

WHITE R. 2001 - Personal Ornaments from the Grotte du Renne at Arcy-sur-Cure. Athena Review, 2 (4), p. 41-46.

ZILHÃO J. 2001 - Anatomically Archaic, Behaviorally Modem The Last Neanderthals and Their Destiny. Amsterdam : Stichting Nederlands Museum voor Anthropologie en Praehistoriae, $93 \mathrm{p}$.

ZILHÃO J. 2006 - Neandertals and Moderns Mixed, and It Matters. Evolutionary Anthropology, 15, 183-195.

ZILHÃO J. 2007 - The emergence of ornaments and art: an archaeological perspective on the origins of behavioural "modernity". Journal of Archaeological Research, 15, p. 1-54. 
ZILHÃO J., D'ERRICO F. 1999 - The chronology and taphonomy of the earliest Aurignacian and its implications for the understanding of Neanderthal extinction. Journal of World Prehistory, 13 (1), p. 1-68.
ZILHÃO J., D'ERRICO F., BORDES J.-G., LENOBLE A., TEXIER J.-P., RIGAUD J.-Ph. 2006 - Analysis of Aurignacian interstratification at the Châtelperronian-type site and implications for the behavioral modernity of Neandertals. Proceedings of the National Academy of Sciences USA, 103 (33), p. 12643-12648. 